\title{
EFFECTS OF SPECIES DIFFERENCES ON OOCYTE REGULATION OF GRANULOSA CELL PROLIFERATION
}

\section{Jia Yi Lin}

A thesis submitted to the Victoria University of Wellington in partial fulfilment of the requirements for the degree of Doctor of Philosophy

Victoria University of Wellington 


\section{Table of Contents}

Content

Page number

ACKNOWLEDGEMENTS VII

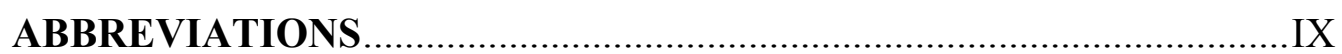

LIST OF TABLES AND FIGURES ..................................................

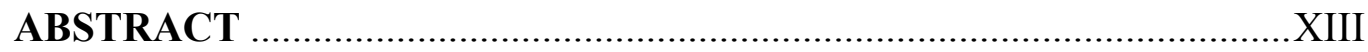

\section{Chapter 1 INTRODUCTION}

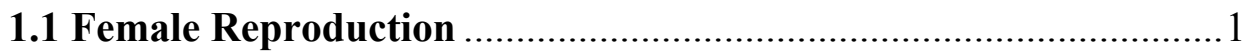

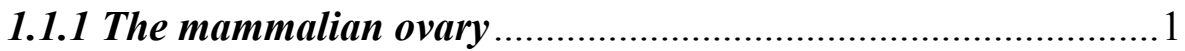

1.1.2 The ovarian follicle .............................................................. 3

1.1.2.1 Follicular formation ............................................................ 3

1.1.2.2 Follicular structure and development ...................................5

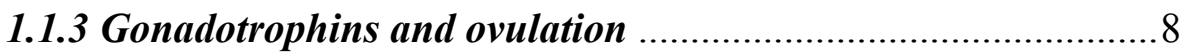

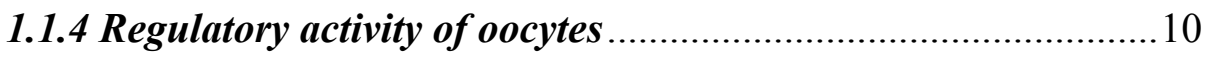

1.2 Transforming growth factor-beta superfamily ...........................12

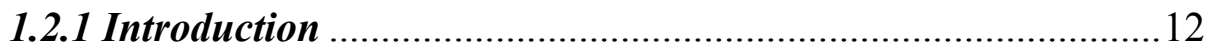

1.2.2 General TGF- $\beta$ signalling mechanism .................................13

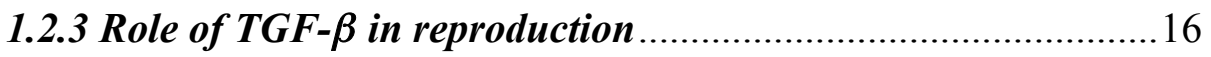

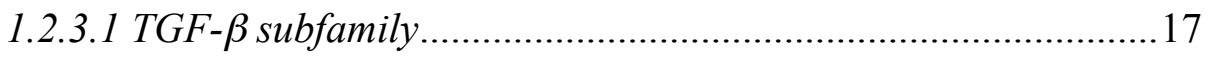

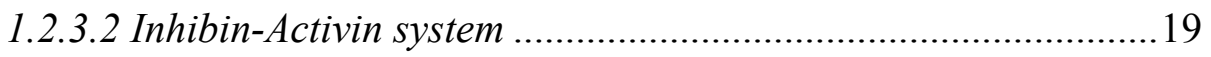

1.2.3.3 Anti-Mullerian Hormone ..........................................................23

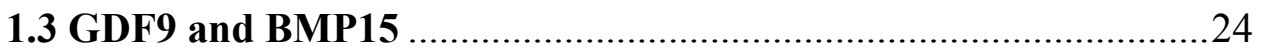




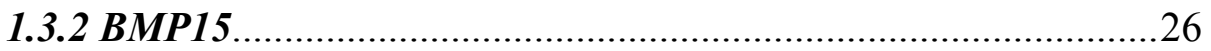

1.3.3 Role of GDF9 and BMP15 in reproduction ...........................29

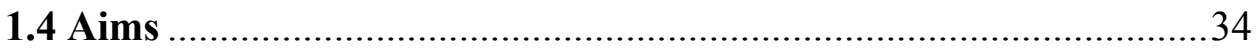

\section{Chapter 2 GENERAL METHODOLOGY}

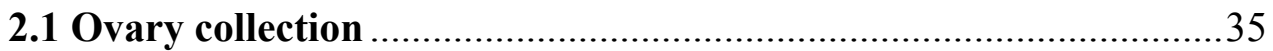

2.2 Oocyte and GC collection and preparation...................................36

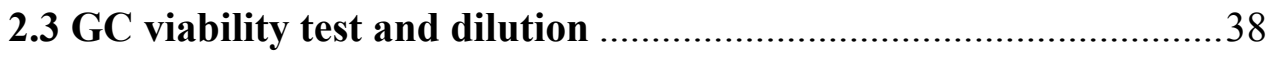

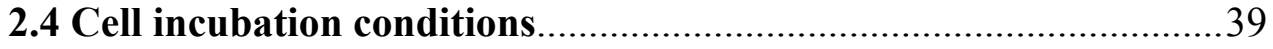

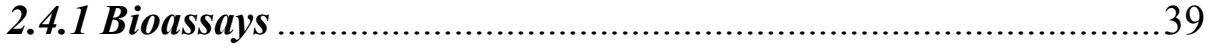

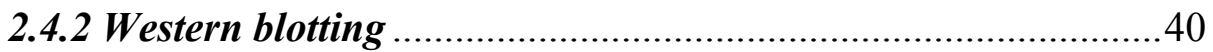

2.5 Methyl- ${ }^{3} \mathrm{H}-$ thymidine incorporation assays ............................... 41

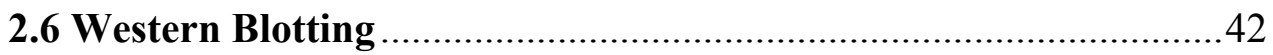

2.7 Collection of COC or DO for the time series studies.....................45

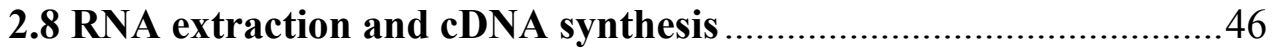

2.9 Real time quantitative PCR (QPCR) ….....................................4

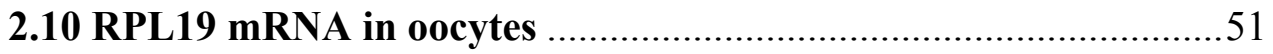

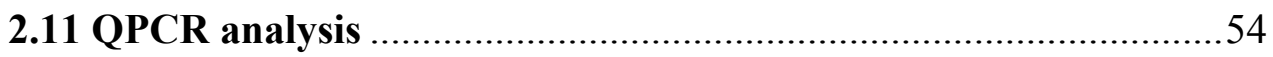

Chapter 3 EFFECTS OF OOCYTE CO-INCUBATION WITH GC ON GC PROLIFERATION IN RAT AND SHEEP

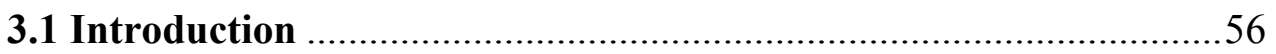

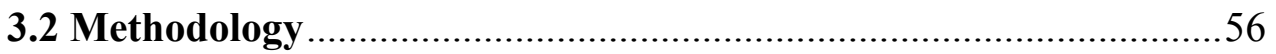


3.3 Results

3.4 Conclusion .60

Chapter 4 CAN OOCYTES FROM A HIGH OVULATION-RATE SPECIES STIMULATE GC PROLIFERATION FROM A LOW OVULATION-RATE SPECIES AND VICE VERSA?

4.1 Introduction .63

4.2 Methodology .64

4.3 Results .65

4.4 Conclusion .69

Chapter 5 EFFECT OF IMMUNO-NEUTRALISATION OF OOCYTE-DERIVED GDF9 OR BMP15 ON ${ }^{3} \mathrm{H}-$ THYMIDINE INCORPORATION BY GC

5.1 Introduction .71

5.2 Methodology .72

5.2 Results .74

5.4 Conclusion .76

Chapter 6 MOLECULAR FORMS OF GDF9 AND BMP15 PROTEINS IN OOCYTE LYSATES AND SPENT MEDIA

6.1 Introduction .78 
6.2 Methodology

6.3 Results

6.4 Conclusion

\section{Chapter 7 EFFECT OF TIME IN INCUBATION ON GDF9}

AND/OR BMP15 mRNA EXPRESSION IN OOCYTES

7.1 Introduction

7.2 Methodology .92

7.3 Results .94

7.4 Conclusion 100

\section{Chapter 8 EFFECT OF TIME IN INCUBATION ON GENE}

\section{EXPRESSION IN CUMULUS CELLS}

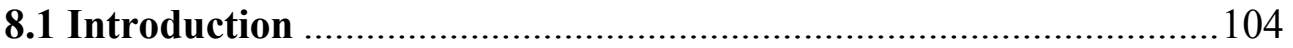

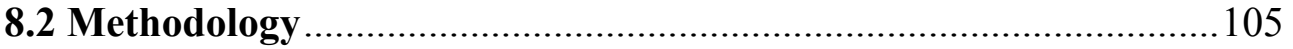

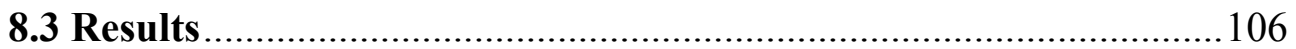

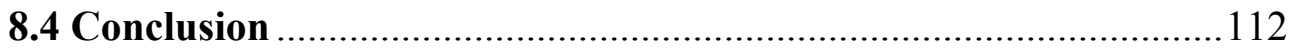

\section{Chapter 9 DISSCUSSION}

9.1 Introduction

9.2 Oocytes stimulate ${ }^{3} \mathrm{H}$-thymidine incorporation in GC of the same species but not necessarily in GC derived from a different species 
9.3 GDF9 and BMP15 are responsible for oocyte stimulatory

effects on ${ }^{3} \mathrm{H}$-thymidine incorporation in GC

9.4 Rat and sheep oocytes show different BMP15 expression

patterns

9.5 CC genes and incubation conditions.

9.6 Conclusion

\section{Appendix 1 MATERIALS}

A1.1 Stock Solutions 131

REFERENCES 138 


\section{ACKNOWLEDGMENTS}

First and foremost my deepest thanks and gratitude to my supervisors Dr Jenny Juengel and Professor Ken McNatty for their patience, guidance and invaluable advice throughout my $\mathrm{PhD}$. This has been an incredible opportunity to work with two amazing supervisors and I have learnt so much from them. They have allowed me to grow not only as a researcher but also as a person and have provided fantastic support throughout. For this opportunity, I am eternally grateful.

Many thanks to the Reproduction group at AgResearch and the staff at the VUW SBS Reproduction group. In particular Stan Lun, Derek Heath and Steve Lawrence for teaching me the experimental protocols and for the continued support throughout my project. Special thanks to Janet Crawford for her patience, advice and help with the QPCR techniques. Also many thanks to Adrian Bibby, Joy McIntosh and Norma Hudson for their advice on Western blotting, molecular biology and culture techniques. And thank you Doug Eckery for great advice and conversations.

My thanks also to my fellow students in the VUW SBS Reproduction group. Thank you for the many coffees breaks, and for always feeding me. Thank you for the great many funny and sometimes very strange conversations, but above all thank you for being my friends.

Finally, my thanks to my family and friends. I would like to thank my parents for their support and especially my Dad for his advice and giving me the drive to finish. 
I'd also like to acknowledge and thank AgResearch for awarding me the AgResearch PhD scholarship. 


\section{ABBREVIATIONS}

ActRII/B

ACVRIB

ALK

$\mathrm{AMH}$

AMHRII

Arom

BMP

BMPRIB

BMPRII

COC

$\mathrm{CC}$

CPM

CREB

Cx43

CycD2

DO

ECD

FGF

FSH

FSHR

GC

GDF

GnRH
Activin receptor type II or type IIB

Activin receptor type IB

Activin receptor-like kinase

Anti-Mullerian hormone

AMH-specific type II receptor

Aromatase

Bone morphogenetic protein

BMP receptor type IB

BMP receptor type II

Cumulus-oocyte-complex

Cumulus cells

Counts per minute

cAMP regulatory element-binding protein

Connexin 43

Cyclin D2

Denuded oocytes

Ectodomain

Fibroblast growth factors

Follicle stimulating hormone

FSH receptor

Granulosa cells

Growth differentiation factor

Gonadotrophin-releasing hormone 
GnRH-R

KITL

$\mathrm{KO}$

LH

LHR

$\mathrm{Mab}$

MAPK

OSFs

PKA

QPCR

SARA

Smad

TC

TF

TGF- $\beta$

TGF- $\beta$ RI

TGF- $\beta$ RII

TS

WT
GnRH receptor

Kit ligand

Knockout

Luteinising hormone

LH receptor

Monoclonal antibody

Mitogen-activated protein kinase

Oocyte-secreted factors

Protein kinase A

Quantitative real time PCR

Smad anchor for receptor activation

Similar to mothers against decapentaplegic

Theca cells

Transcription factor

Transforming growth factor-beta

TGF- $\beta$ receptor I

TGF- $\beta$ receptor II

Time series

Wild type 


\section{LIST OF TABLES AND FIGURES}

Tables

Page number

$1 \mathrm{~A}$

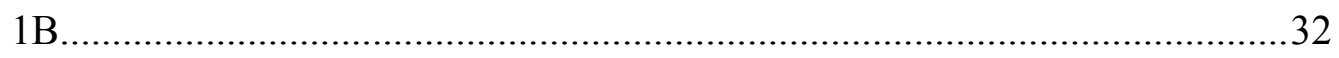

$2 \mathrm{~A}$

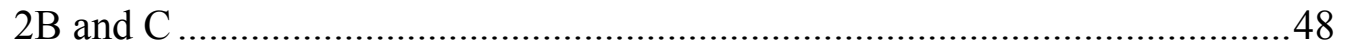

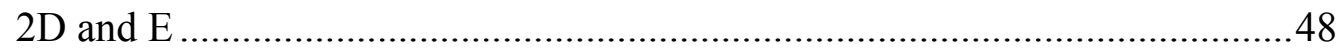

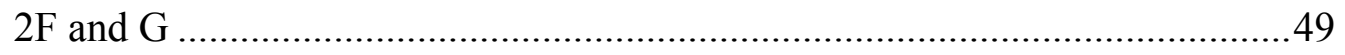

$2 \mathrm{H}$

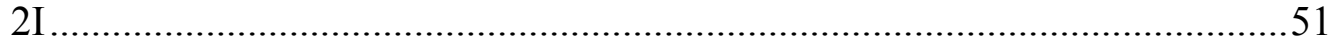

2J

5А

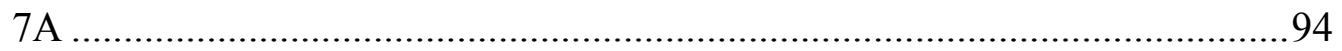

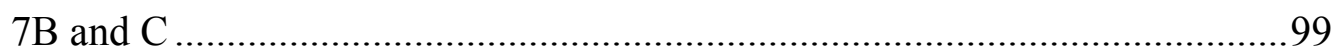

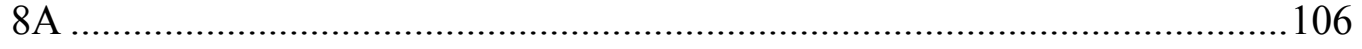

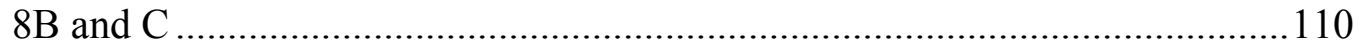

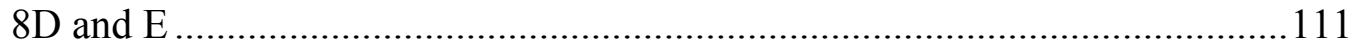

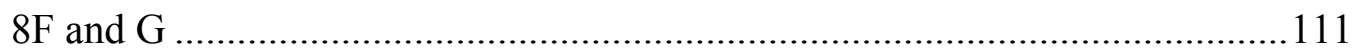

$\begin{array}{ll}\text { Figures } & \text { Page number }\end{array}$

$1 \mathrm{~B}$




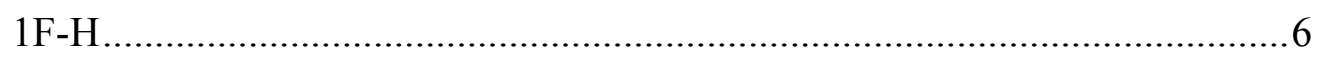

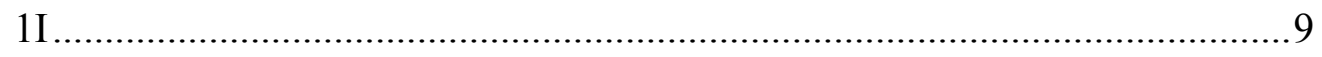

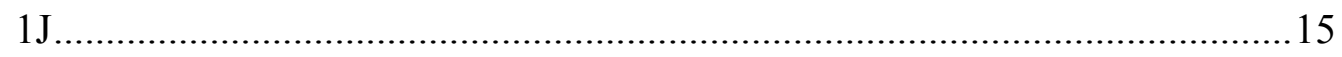

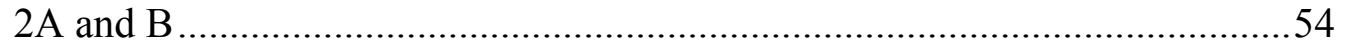

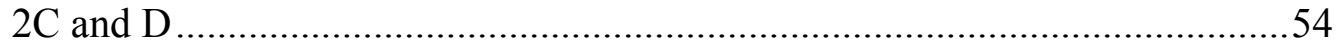

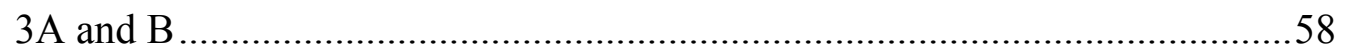

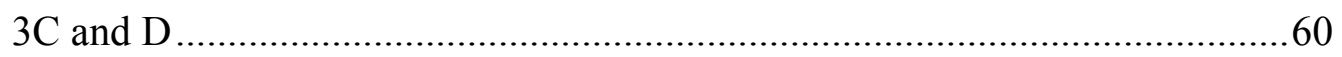

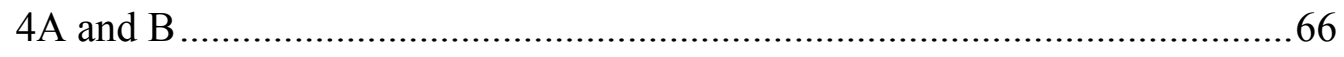

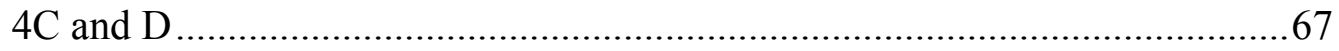

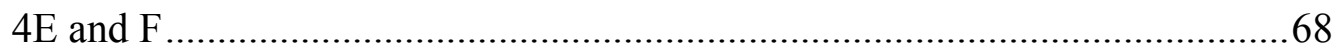

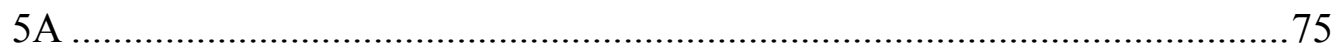

5В

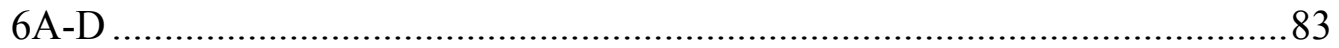

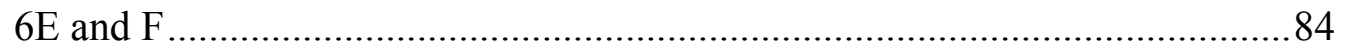

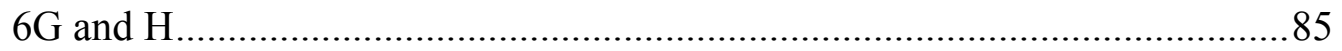

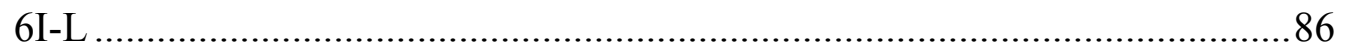

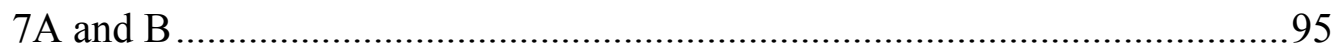

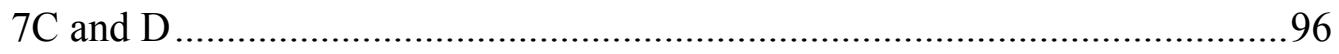

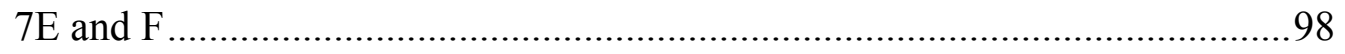

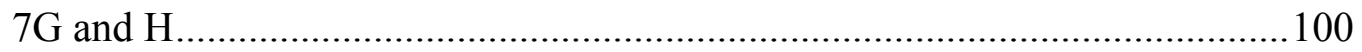

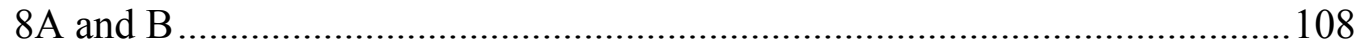

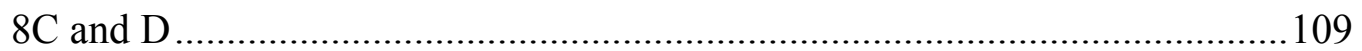




\begin{abstract}
The role of oocytes in regulating ovulation quota between species is not fully understood. In humans, sheep, and rodents the oocyte-derived growth factors, bone morphogenetic protein 15 (BMP15) and/or growth differentiation factor 9 (GDF9) have profound effects on ovarian follicular development and ovulation quota. The aim of these studies was to compare the ability of oocytes from sheep (low ovulation-rate) and rat (poly-ovulator) to stimulate radiolabelled ${ }^{3} \mathrm{H}$-thymidine uptake by granulosa cells (GC) both within and between the two species, and to assess species differences of GDF9 and BMP15 in co-incubations. For these experiments, oocytes denuded of cumulus-cells (DO) were co-incubated with a fixed number of GC from either species.
\end{abstract}

Rat or sheep DO stimulated ${ }^{3} \mathrm{H}$-thymidine uptake by GC from the same species $(\mathrm{P}<0.005)$. Sheep oocytes also stimulated ${ }^{3} \mathrm{H}$-thymidine uptake by rat $\mathrm{GC}(\mathrm{P}<0.001)$ but not vice versa. To investigate this further, oocytes and GC were co-incubated with monoclonal antibodies specific to GDF9 or BMP15 or to a hydatids antigen (control). Both sheep and rat oocyte stimulation of ${ }^{3} \mathrm{H}$-thymidine uptake by GC was inhibited with the GDF9 antibody $(\mathrm{P}<0.05)$ but not the control antibody, irrespective of the species of GC. Sheep DO stimulation of rat GC was also inhibited using an antibody to BMP15 $(\mathrm{P}<0.05)$. However, when using the BMP15 antibody to block the effects of rat DO on rat GC, no inhibition of ${ }^{3} \mathrm{H}$-thymidine uptake was observed $(\mathrm{P}=0.988)$.

The molecular forms of GDF9 and BMP15 protein in oocyte lysates and spent media were examined by Western blotting under reducing conditions. For both species, 
GDF9 protein was present in the mature form in both the lysate and the spend media. For sheep oocytes, BMP15 protein was present as pro-mature and monomeric mature forms in the lysate and in the spent media, whereas from rats pro-mature forms were detected in the oocyte lysate but there was minimal evidence for the mature form in the spent media.

The mRNA levels of GDF9, BMP15 and several cumulus cell (CC) genes were measured at $8 \mathrm{~h}$ intervals over a $24 \mathrm{~h}$ incubation period. For both sheep and the rat, GDF9 and BMP15 mRNA expression levels were highly correlated $\left(\mathrm{R}^{2}=0.99\right)$. In the rat, the relative mean expression level of $G d f 9$ mRNA was four times higher than Bmp15 mRNA, whereas in sheep the relative mean expression ratio of GDF9 to BMP15 was approximately one. For these studies, two types of incubations were performed, namely COC alone or co-incubations of DO with GC. In co-incubations of DO with GC, the relative GDF9 and BMP15 mRNA levels did not change significantly over time, whereas incubations of COC showed that the relative levels of GDF9 and BMP15 mRNA declined significantly during the incubation period. The CC genes (FSHR, LHR, KITL, CX43, AROM and CYCD2) were measured during the 24h incubation period for the COC but not the DO experiment. In rats, none of the CC genes showed any changes in mRNA levels over time except Kitl, where levels at $8 \mathrm{~h}$ and $16 \mathrm{~h}$ mRNA levels were significantly lower compared to those at $0 \mathrm{~h}$ and $24 \mathrm{~h}$. In sheep, there were no significant mRNA changes in any of the $\mathrm{CC}$ genes except $A R O M$. The level of $A R O M$ mRNA reduced to near zero by $8 \mathrm{~h}$ and remained at low levels at both $16 \mathrm{~h}$ and $24 \mathrm{~h}$. These findings suggest that, under the incubation conditions used, that the gene expression levels for most CC genes were maintained 
but that the oocyte growth factors alone were unable to maintain $A R O M$ gene expression in sheep.

In conclusion, major differences were observed in GDF9 and BMP15 expression and regulation between rats and sheep. The lower expression levels of Bmp15 mRNA and protein are likely the reason why rat DO failed to stimulate ${ }^{3} \mathrm{H}$-thymidine uptake by sheep GC. The evidence suggests that oocyte-derived GDF9 is sufficient to stimulate ${ }^{3} \mathrm{H}$-thymidine incorporation and presumably DNA synthesis in rat GC whereas in sheep both BMP15 and GDF9 are required. These findings raise the possibility that in species with a low ovulation rate phenotype there is a higher level of GDF9 mRNA and protein synthesis and that BMP15 is a major factor in restricting ovulation quota in mammals. 


\section{Chapter 1 INTRODUCTION}

\subsection{Female Reproduction}

\subsubsection{The mammalian ovary}

The mammalian ovary is enclosed within a layer of epithelium known as the surface epithelium (Peters and McNatty 1980). When viewing the surface of the ovary, ovarian follicles are often visible as fluid-filled entities (Figure 1A). Within the ovary, the outermost region is known as the ovarian cortex (Figure 1B and C). The ovarian cortex contains dense connective tissue regions, which are required to support the extensive numbers of ovarian follicles that reside within this layer (Peters and McNatty 1980). Each follicle within the cortex contains an egg (i.e. the oocyte) surrounded by one or more layers of somatic cells (Figures 1D). Below the cortex is the medulla, which contains the main blood supply and lymphatic vessels (Figure 1B and C).

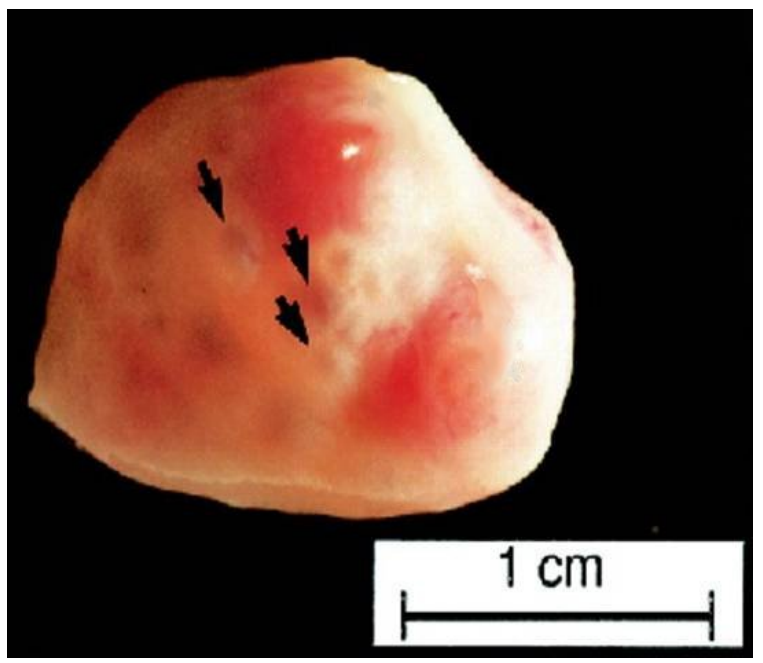

Figure 1A: A sheep ovary showing fluid-filled follicles (black arrows) (modified from Juengel, Quirke et al. 2000). 


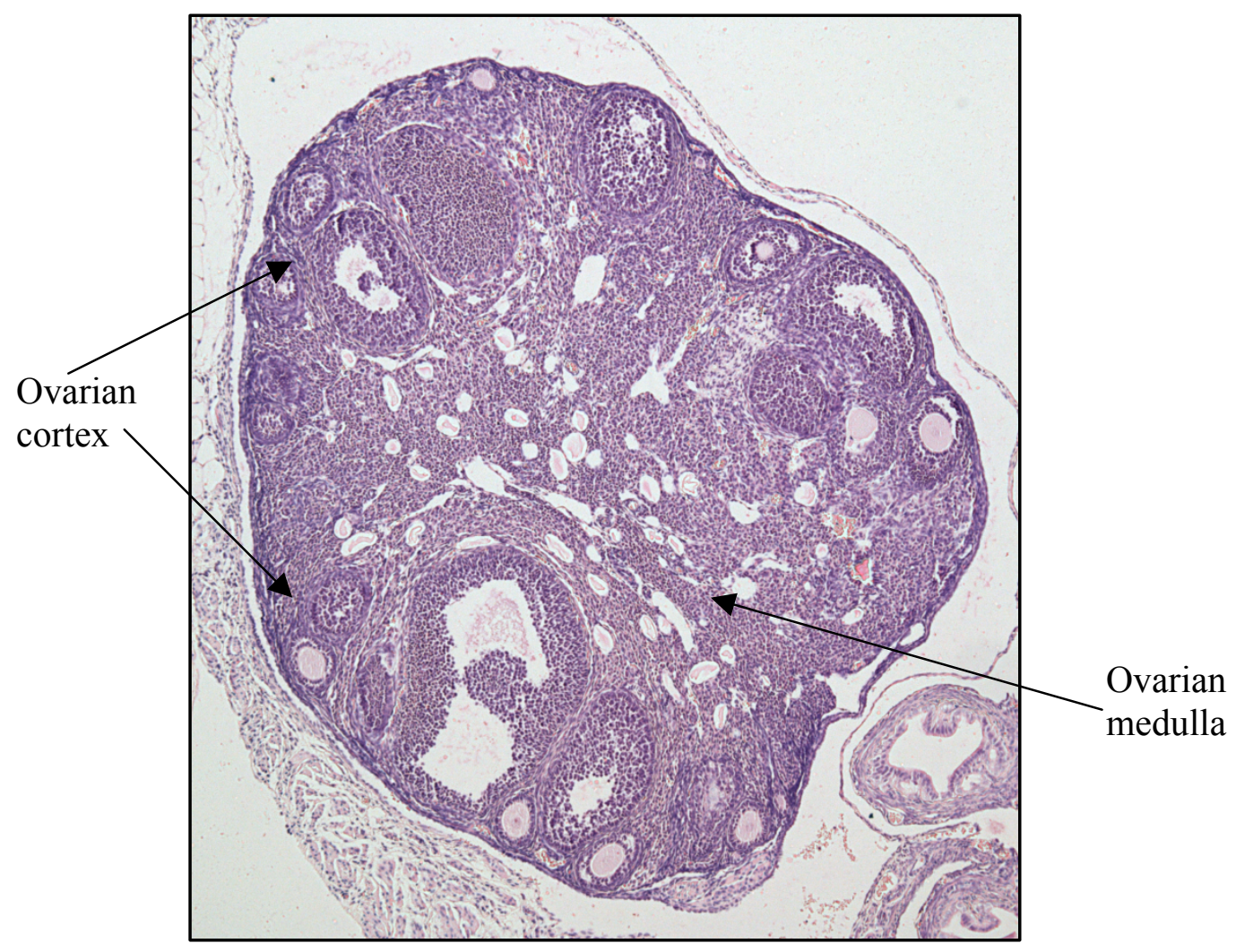

Figure 1B: Cross-section of a 5.6-month-old mouse ovary. Image supplied by Dr J Crawford.

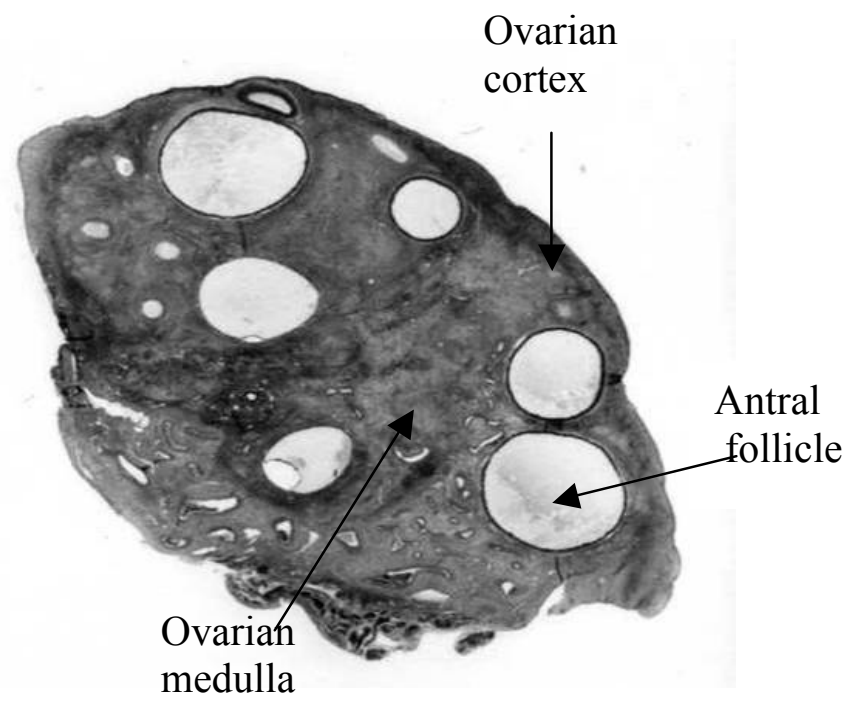

Figure 1C: Cross-section of a sheep ovary showing numerous antral follicles and small antral follicles (modified from Juengel, Hudson et al. 2002). 


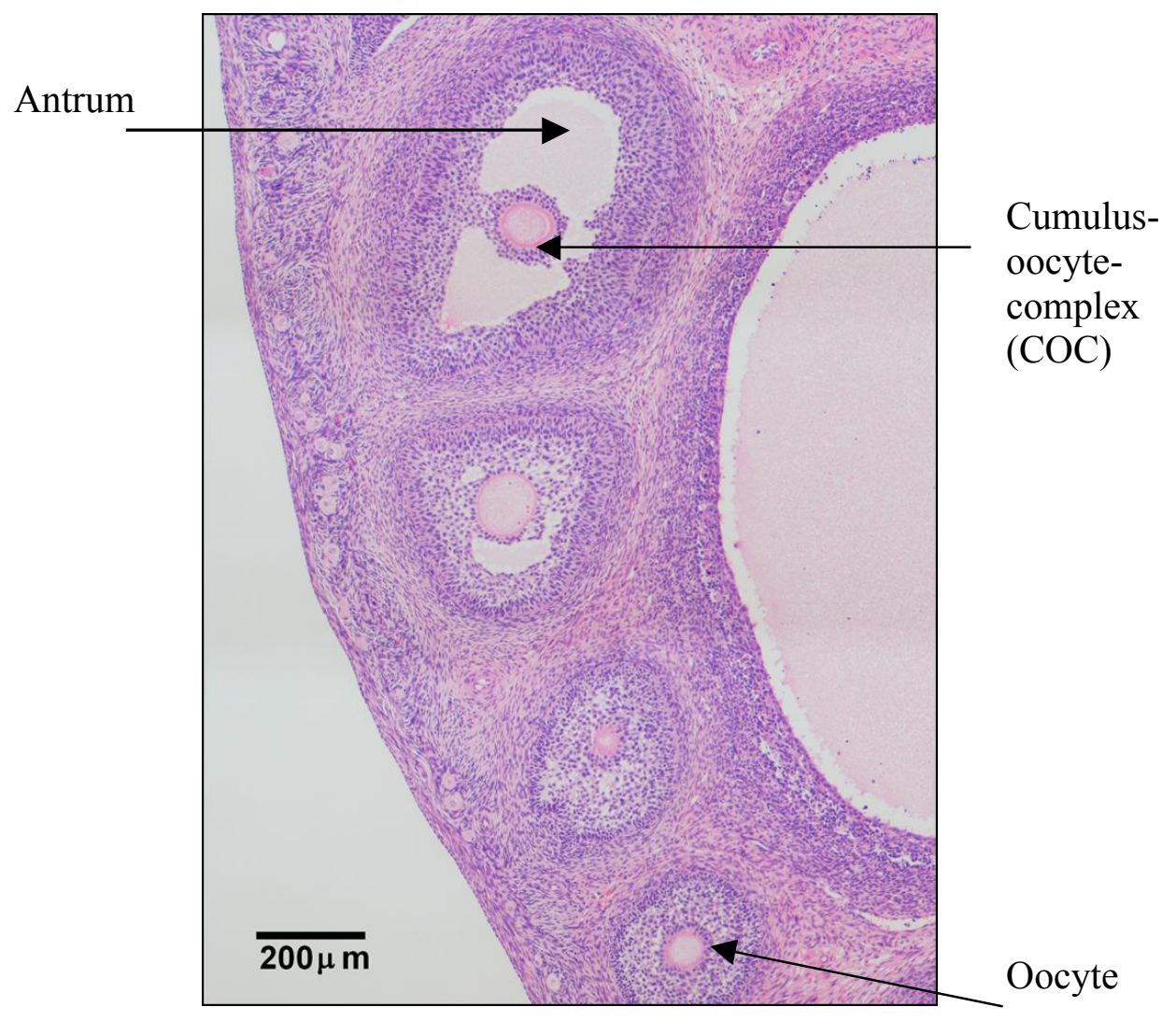

Figure 1D: Four follicles at sequential stages of folliculogenesis. The bottom-most follicle is a preantral follicle followed by a follicle just starting to form an antrum. Next a small antral follicle and the top-most follicle has a well-developed antrum. The large fluid filled area on the right hand side represents a partly obscured large antral follicle. Image supplied by Prof K McNatty.

\subsubsection{The ovarian follicle}

\subsubsection{Follicular formation}

In many mammalian species (e.g. sheep, cattle, and primates including humans), follicular formation involves migration of germ cells from the hindgut to the developing gonad during early foetal life. Then, after sexual differentiation, mitotically active oogonia attach to intraovarian mesenchymal cells and later to somatic cells of the surface epithelium to form oogonia-pregranulosa cell entities (van Wagenen and Simpson 1965; Sawyer, Smith et al. 2002). Progressively, the pregranulosa cells secrete a basal lamina enclosing the clusters of oogoniapregranulosa cells to form ovigerous cords which in sheep occurs after day 38 of foetal life (Figure 1E) (Sawyer, Smith et al. 2002). Ovigerous cords are characterised 
by a basal lamina enclosing the oogonia-pregranulosa cells which separates them from the surrounding ovarian tissue. However, the ovigerous cords remain open to the surface epithelium at least until day 100 in sheep and day 230 in humans (van Wagenen and Simpson 1965; Sawyer, Smith et al. 2002). Within these cords, the oogonia enter meiosis to form oocytes. During this transition many $(>80 \%)$ of the meiotic germ cells, but not the pregranulosa cells, undergo apoptosis (Baker 1963; Sawyer, Smith et al. 2002). Thus in addition to pregranulosa cells being recruited by proliferating oogonia from the surface epithelium, the surviving meiotic oocytepregranulosa cell entities recruit additional pregranulosa cells from those oogonia that degenerated while attempting to enter meiosis (van Wagenen and Simpson 1965; Sawyer, Smith et al. 2002).

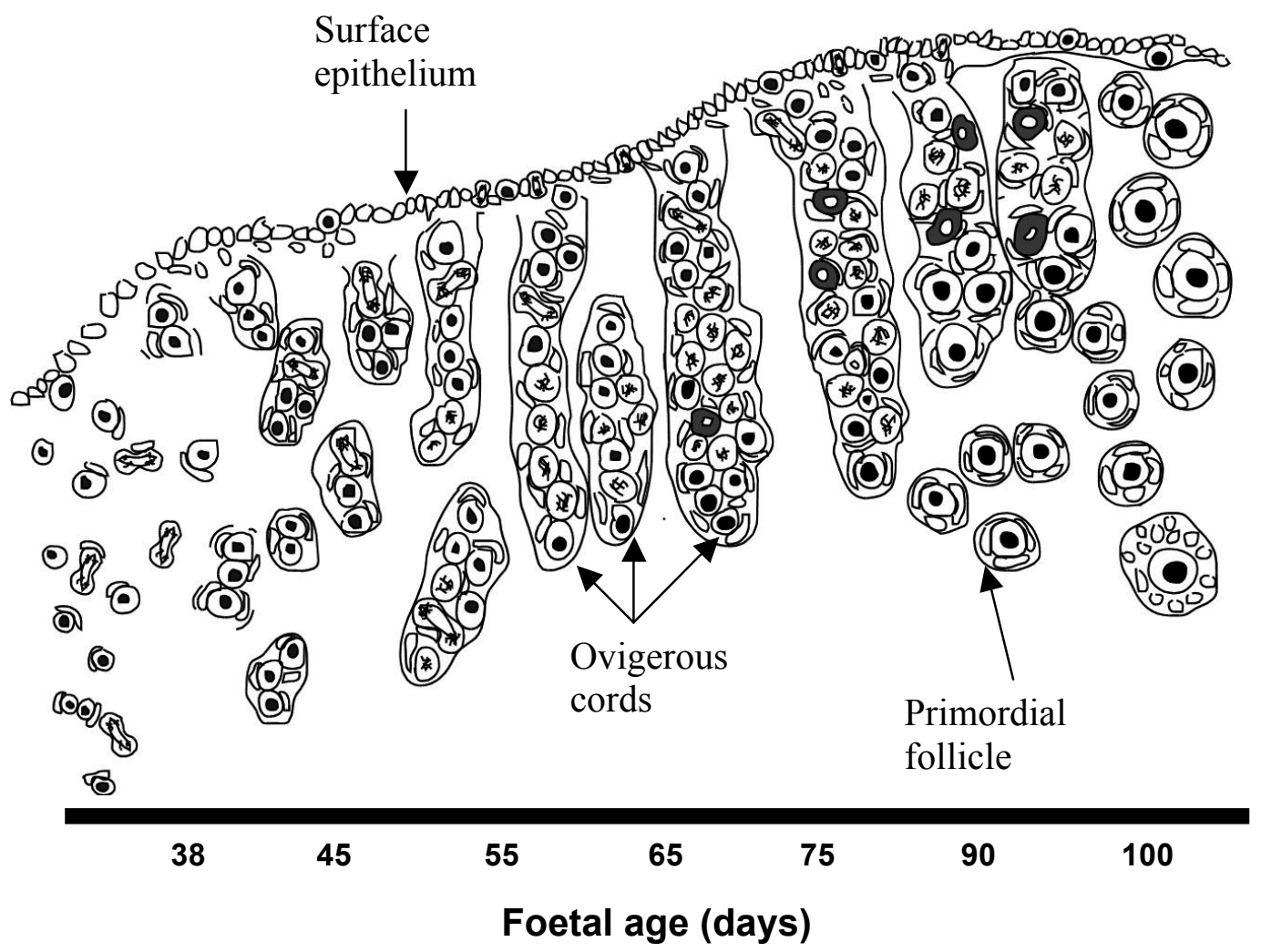

Figure 1E: Schematic outline of the ontogeny of follicular formation during foetal life in sheep. Dark cells within the ovigerous cords with a clear centre are degenerate germ cells (modified from Juengel, Sawyer et al. 2002). 
From the base of ovigerous cords, the oocyte-pregranulosa cell entities begin to separate from one another by secreting a basal lamina. This process begins after day 75 and 130 of foetal life in sheep and humans respectively (Figure 1E) (McNatty, Quirke et al. 2000; Sawyer, Smith et al. 2002). In the newly formed follicles (known as primordial follicles), the meiotic oocytes are arrested at the diplotene stage of meiosis I (Figure 1F). The resumption of meiosis does not resume again until the ovarian follicle has developed to near ovulation and in response to the pre-ovulatory rise in luteinising hormone (LH), which triggers the sequence of events leading to ovulation and fertilization of the oocyte (McGee and Hsueh 2000).

\subsubsection{Follicular structure and development}

Primordial follicles are characterised by an immature oocyte enveloped within a single layer of squamous granulosa cells (GC) (Figures 1F and G) (Lundy, Smith et al. 1999; McGee and Hsueh 2000). At any moment in time, most follicles are present as primordial follicles (Peters, Byskov et al. 1975). However, shortly after the first follicles form, some begin to grow towards ovulation. Thus, even before birth, in many species, the process of follicular growth is initiated. Once the initiation of follicular growth begins, it continues without rest until follicles die or ovulate (Peters, Byskov et al. 1975). The process of follicular growth continues throughout life uninterrupted by reproductive state (i.e. pre-pubertal, pregnancy, anoestrus etc.) until the pool of primordial follicles is depleted (woman at menopause) or death of the animal (sheep) (McGee and Hsueh 2000). 
Follicles develop in sequential stages from the pool of primordial follicles (Figure 1D) (Peters, Byskov et al. 1975; McGee and Hsueh 2000). As primordial follicles develop to primary follicles, the GC change their shape to become uniformly cuboidal and thereafter proliferate in multiple layers (see Figures 1F and H) (Lundy, Smith et al. 1999). Concurrently, a layer of glycoprotein is formed around the oocyte and is known as the zona pellucida (Lundy, Smith et al. 1999; Senbon, Hirao et al. 2003). Somatic cells associated with the follicle but outside the basement membrane differentiate into two layers: the theca interna (innermost layer) and the theca externa (Lundy, Smith et al. 1999). Unlike the avascular GC, theca cells are adjacent to blood capillary networks (Reynolds 1973).
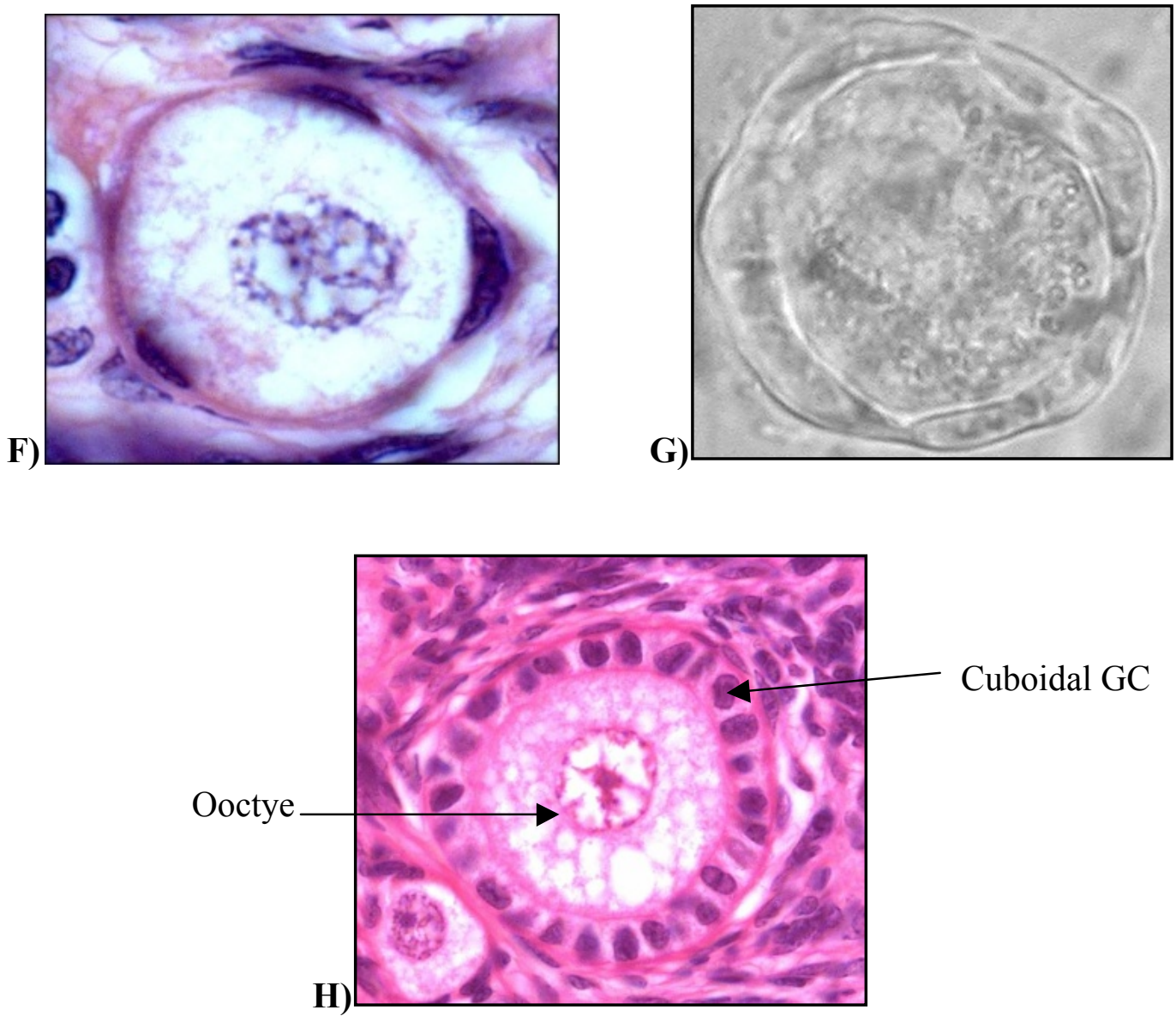

Figure 1F-H: A primordial follicle with three clearly visible GC (F). An isolated primordial follicle as seen under an inverted phase contrast microscope (G) and a primary follicle with a single layer of cuboidal GC (H). Images supplied by Prof K McNatty. 
As follicular growth progresses, the proliferating $\mathrm{GC}$ secrete proteoglycans leading to fluid accumulation within the follicle and the formation of fluid-filled spaces. Eventually, these fluid-filled spaces coalesce to form a single fluid-filled cavity known as the antrum (Figure 1D) (Peters and McNatty 1980; Rodgers and IrvingRodgers 2010). The antrum contains many blood-derived products including amino acids together with follicular secretions and metabolites and many of these factors are required by the oocyte to continue development (Thompson, Lane et al. 2007). Formation of the antrum results in physical changes in the GC populations which develop into two phenotypes. The GC immediately associated with the oocyte differentiate into specialised cumulus cells (CC) and become tightly attached to each other and the oocyte, forming the cumulus-oocyte-complex (COC) (Figure 1D) (Gilchrist, Ritter et al. 2004; Sugiura and Eppig 2005). The GC more distant from the oocyte and closer to the basement membrane are referred to as the mural granulosa cells (MGC). Concurrent with this process, is a progressive increase in volume of the oocyte, and likely an increasingly greater requirement for nutrients and substrates. From the primordial stage, the oocyte undergoes a 500 fold increase in volume and a four to six fold increase in diameter (Peters and McNatty 1980). Those follicles that have developed an antrum are termed antral follicles (Figures 1A-D). Early follicular growth from the primordial stage depends largely on signals from within the ovary (Nakano, Mizuno et al. 1975; Peters and McNatty 1980; McNatty, Lawrence et al. 2006). As the follicle grows from the pre-antral to antral stage, its continued growth becomes increasingly dependent on gonadotrophic signals from the anterior pituitary gland, specifically in the form of LH and follicle stimulating hormone (FSH) (Scaramuzzi, Adams et al. 1993; Messinis, Messini et al. 2010). 


\subsubsection{Gonadotrophins and ovulation}

Gonadotrophins belong to a glycoprotein family of pituitary proteins. Members of this family share a common alpha subunit and each have a unique beta subunit, which confers the hormone specificity. The two most important pituitary hormones regulating mammalian female fertility are $\mathrm{LH}$ and $\mathrm{FSH}$.

The LH protein shares a common alpha subunit with FSH and a unique beta subunit (Pierce and Parsons 1981). The pituitary releases LH in a sequence of episodic pulses. During the follicular phase of an ovarian cycle, these LH pulses increase in frequency until a major surge occurs leading to peak levels of LH triggering the rupture of the pre-ovulatory follicles and release of mature oocytes into the reproductive tract (see reviews, (Scaramuzzi, Adams et al. 1993; Messinis, Messini et al. 2010).

Episodic release of gonadotrophin-releasing hormone $(\mathrm{GnRH})$ by the hypothalamus (Figure 1I; box 1 and 2) stimulates the synthesis and release of FSH and LH from the anterior pituitary gland. Pulses of LH together with waves of FSH stimulate responsive follicles to develop into pre-ovulatory follicles and to synthesize and secrete steroid hormones, such as androstenedione and oestradiol (Figure 1I; box 3, 4 \& 5) (Baird, Swanston et al. 1981; Bäckström, McNeilly et al. 1982; Scaramuzzi, Adams et al. 1993; McGee and Hsueh 2000; Russell and Robker 2007). MGC and CC are the only cells within the ovary that have receptors for FSH. In contrast, LH receptors are initially present only in theca interna cells but during pre-ovulatory follicular maturation they are also present in the MGC, with little or no expression in 
the CC (Webb and England 1982; Peng, Hsueh et al. 1991; McGee and Hsueh 2000; Russell and Robker 2007).

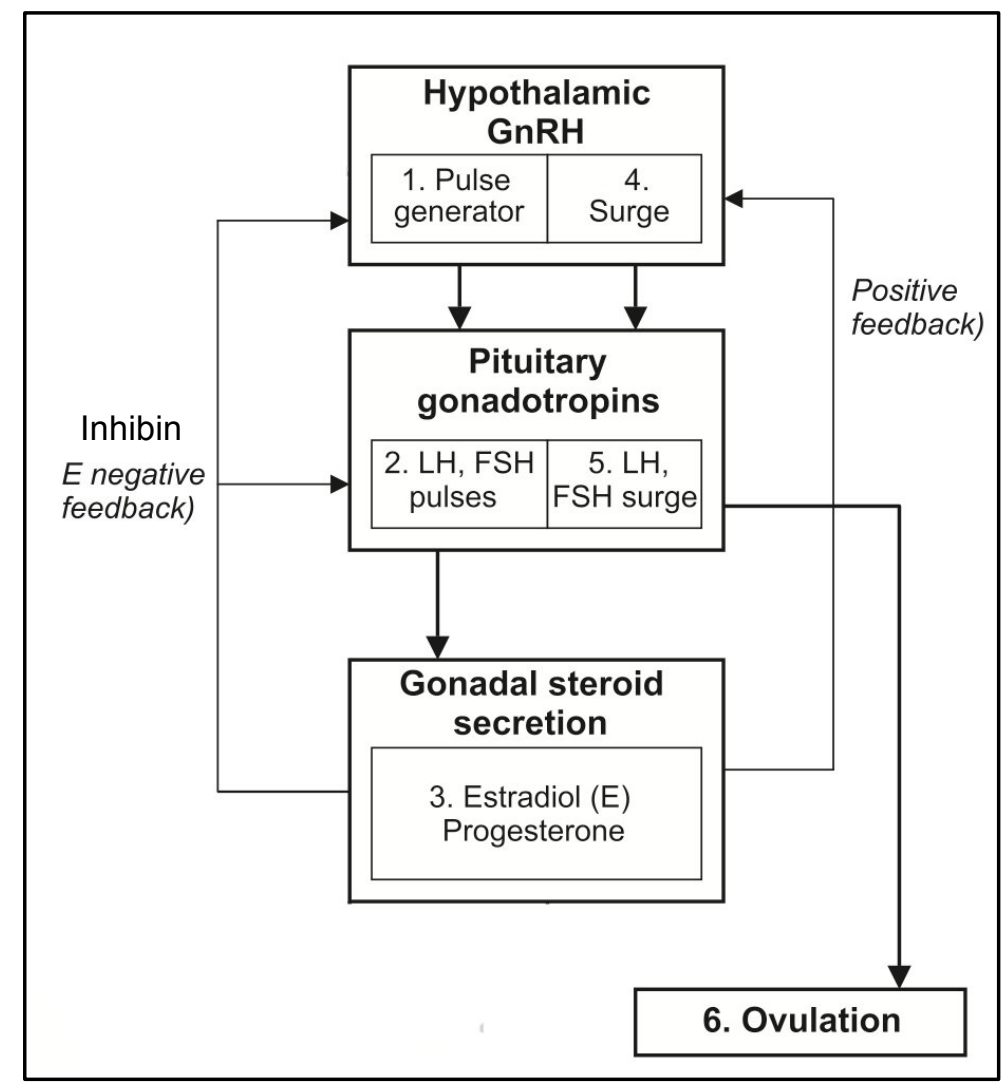

Figure 1I: Hypothesised relationship between the various points in the regulation of fertility under normal conditions (modified from Bilezikjian, Blount et al. 2006 and Schneider 2004).

As the gonadotrophin-receptive follicles grow, a restricted number emerge as the presumptive pre-ovulatory follicles that go on to ovulate. In a low ovulation-rate phenotype (e.g. sheep, humans, cattle) the number of pre-ovulatory follicles is generally limited to one or two whereas in a high ovulation-rate phenotype (e.g. rodent) this number may vary between six and sixteen. Oestradiol-17ß secreted by the pre-ovulatory follicle(s) has a negative feedback effect on FSH secretion (Figure 1I) (Peters and McNatty 1980; Baird, Swanston et al. 1981; Scaramuzzi, Adams et al. 1993; Schneider 2004). Less-developed follicles, which are still dependent on FSH for continued growth, become atretic and die due to the decreasing concentrations of 
FSH. The pre-ovulatory follicle(s), however, acquires LH receptors in the MGC that respond to the increasing pulses of $\mathrm{LH}$ to continue growth and increase its secretion of oestradiol-17ß (Schneider 2004). As oestradiol-17ß secretion increases, the rapid rise in concentration exerts a positive feedback loop on the hypothalamus and the pituitary to release more $\mathrm{LH}$, which culminates in the LH surge to trigger ovulation (Figure 1I; boxes 4, 5 and 6) (Russell and Robker 2007).

\subsubsection{Regulatory activity of oocytes}

It was traditionally thought that oocytes acquired metabolic products passively via gap junctions. However, it is now thought that the oocyte is active in regulating its nutritional status and hence influences its own development by actively regulating $\mathrm{CC}$ functions (Eppig 2001; Senbon, Hirao et al. 2003).

It has been known for a long time that in vitro culture of oocytes stripped of its cumulus cells, known as denuded oocytes (DO), were very poor at glucose uptake and lacked expression of genes involved in glycolysis although they were able to metabolise pyruvate (Senbon, Hirao et al. 2003; Sugiura, Pendola et al. 2005). In contrast, under in vitro conditions, COC were able to utilise glucose as well as metabolizing glucose to pyruvate (Sutton, Cetica et al. 2003; Sugiura, Su et al. 2007; Thompson, Lane et al. 2007). This indicates that CC are able to take up glucose and metabolise this substrate into intermediate products for ultimate use by the oocyte. Studies using COC with their ooplasm removed (OOX) have shown that oocytes can influence metabolic activities of CC and the associated enzyme expression levels ( $\mathrm{Li}$, Norman et al. 2000; Sugiura, Pendola et al. 2005; Gilchrist, Ritter et al. 2006). Culturing of these complexes showed that the $\mathrm{CC}$ expressed very low levels of 
glucose metabolism genes compared with intact COC, and showed lower levels of glucose metabolism (Sugiura, Pendola et al. 2005; Gilchrist, Ritter et al. 2006). When DO were co-cultured with OOX complexes, the level of glucose metabolism and associated gene expression levels were restored to values comparable with those in intact COC (Sugiura, Pendola et al. 2005; Gilchrist, Ritter et al. 2006).

Other substrates required by oocytes are also delivered via the CC. (Eppig, Pendola et al. 2005) have shown that certain amino acids must be transported via the $\mathrm{CC}$ as they are not able to enter oocytes directly. For example, when OOX were cultured alone, the level of L-leucine incorporation was low but increased significantly when cocultured with DO (Eppig, Pendola et al. 2005). Thus the ability of CC to transport amino acids to oocytes required the presence of factors produced by oocytes.

High levels of cAMP are required to maintain oocytes at meiotic arrest prior to ovulation. It was thought that cAMP were only synthesised in $\mathrm{CC}$ and then transported to the oocyte via gap junctions (Conti, Andersen et al. 1998; Russell and Robker 2007). However, a recent study by (Vaccari, Horner et al. 2008) suggests that oocytes also participate in cAMP synthesis and in the maintenance of high intracellular cAMP prior to ovulation.

Collectively, the aforementioned results show that there is an interdependent relationship between oocytes and their $\mathrm{CC}$, which suggests that oocytes have an active role in regulating CC functions (Eppig 2001; Eppig, Wigglesworth et al. 2002). While oocytes regulate $\mathrm{CC}$ functions, $\mathrm{CC}$ in turn influence oocyte health and development. For example, maturation of oocytes is dependent on differentiation and function of 
adjacent CC (Gilchrist and Thompson 2007; Webb, Garnsworthy et al. 2007; Scaramuzzi, Baird et al. 2011). During folliculogenesis, oocyte-secreted factors (OSF) promote $\mathrm{CC}$ phenotype differentiation in adjacent cells; the functions of $\mathrm{CC}$ in turn help the oocyte to reach maturation (Vanderhyden, Telfer et al. 1992; Eppig 2001; Gilchrist, Ritter et al. 2004; Eppig, Pendola et al. 2005; Sugiura and Eppig 2005; Sugiura, Pendola et al. 2005; Diaz, Wigglesworth et al. 2007; Gilchrist and Thompson 2007).

It is reasonable to assume that ability of an oocyte to be fertilised and develop into a blastocyst is linked to its ability to mature and acquire developmental competence. Since CC differentiation seems to be a rate-limiting step in oocyte maturation, they clearly play an important role in influencing oocyte quality. However, this relationship is not well understood especially in species other than rodents. Therefore, by investigating in more detail the relationships between the oocyte and companion $\mathrm{CC}$ in different species we are likely to gain new insights into factors influencing oocyte quality.

\subsection{Transforming growth factor-beta superfamily}

\subsubsection{Introduction}

Important regulatory factors involved in the oocyte-CC communication system are hypothesised to be the OSF that function either by autocrine or paracrine actions.

Many of the OSF that have been identified thus far are members of the transforming growth factor-beta (TGF- $\beta$ ) superfamily (Knight and Glister 2006). Members consists of a number of signalling molecules such as those in the growth differentiation factor 
(GDF) subfamily and the bone morphogenetic protein (BMP) subfamily (McPherron and Lee 1993).

These family members have very similar structures, and most form as bioactive homo- or heterodimers (Liao, Moore et al. 2004). Members of this family are synthesised as pre-pro-mature proteins. The pro-regions are hypothesised to be important in ensuring correct folding and dimerisation between mature regions (McPherron and Lee 1993; Liao, Moore et al. 2004). With the exception of GDF9, BMP15, GDF3, lefty1 and lefty2 (McPherron and Lee 1993; Dube, Wang et al. 1998; Oulad-Abdelghani, Chazaud et al. 1998; Hashimoto, Uji et al. 2007), all other members of the TGF- $\beta$ superfamily have seven or nine conserved cysteines, pairs of which enable cysteine knot and disulfide bond formation (McPherron and Lee 1993; Kingsley 1994; Drummond 2005; Knight and Glister 2006). The disulfide bond allows covalent linkage between the mature regions of two TGF- $\beta$ proteins and thus dimerisation. In contrast, due to the lack of one cysteine residue in GDF9, BMP15, GDF3, lefty 1 and lefty2, dimer formations by covalent linkage in these molecules is not possible (Shimasaki, Moore et al. 2004).

\subsubsection{General TGF- $\beta$ signalling mechanism}

Before the role of TGF- $\beta$ superfamily in reproduction is discussed, it is helpful to understand their general signal transduction mechanism. This section explains the mechanism utilised by TGF- $\beta$.

Members of the TGF- $\beta$ superfamily signal via transmembrane serine-threonine receptor kinases. These receptor kinases are classified into two groups: type I and type 
II. So far there are seven type I and five type II receptors that have been identified (Chang, Brown et al. 2002; de Caestecker 2004; Dijke and Hill 2004). Although the exact composition of receptors is not known, it is thought that ligand-receptor complexes consists of two type I and two type II receptors (Chang, Brown et al. 2002; Dijke and Hill 2004). In many cases it is thought that the ligand binds to the type II receptor first and recruits the type I receptor, however, it has been suggested that BMPs may bind a type I receptor first and thereafter recruit the type II receptor (Dijke and Hill 2004; Drummond 2005). The binding affinity and order of binding appear to be influenced by stoichiometry of the ligand and receptors. Upon oligomerisation, type II receptors phosphorylate the glycine and serine rich motif of the type I receptor, known as the GS domain, located near the transmembrane portion of the receptor (Figure 1J) (Dijke and Hill 2004; Mazerbourg and Hsueh 2006). Phosphorylation of type I receptors allows them to propagate a signal by phosphorylating similar to mothers against decapentaplegic (Smad) proteins that are presented to them by auxiliary proteins such as Smad anchor for receptor activation (SARA) proteins (Dijke and Hill 2004). 

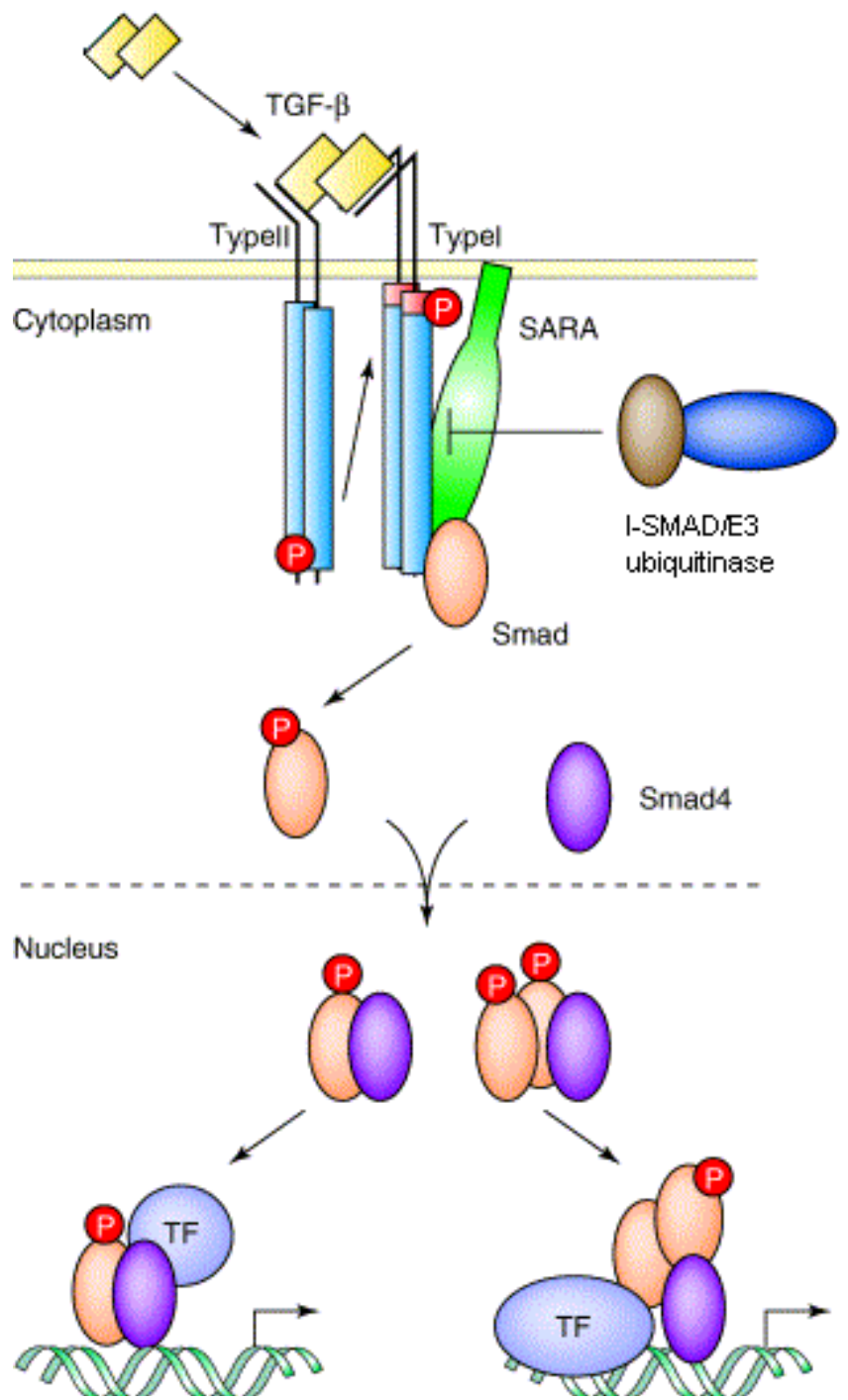

Figure 1J: Schematic representation of the TGF- $\beta$-Smad signalling pathway (modified from Dijke and Hill 2004). $P=$ phosphorylation; Type $I=T$ TF- $\beta$ type I receptor; Type II=TGF- $\beta$ type II receptor; I-Smad=inhibitory Smad; SARA=Smad anchor for receptor activation; $\mathrm{TF}=$ transcription factors.

Phosphorylated Smads form complexes with a common Smad protein, Smad 4, for translocation into the nucleus (Drummond 2005; Mazerbourg and Hsueh 2006). Once in the nucleus, the heteromeric Smad complexes interact with local transcription factors via the Mad Homology 1 (MH1) domain and Mad Homology 2 (MH2) domain (Mazerbourg and Hsueh 2006), which allows Smad complexes to bind to target genes and alter transcription levels (Dijke and Hill 2004; Mazerbourg and Hsueh 2006). 
Inhibition of Smad signalling can be achieved by inhibitory Smad proteins 6 and 7 (Mazerbourg and Hsueh 2006). It is thought they compete with signalling Smads for binding to phosphorylated type I receptors, and are able to recruit E3-ubiquitin ligases to type I receptors resulting in their degradation and thus termination of signal (Dijke and Hill 2004; Mazerbourg and Hsueh 2006).

While the TGF- $\beta$ superfamily consists of many members, Smad signalling only consists of two pathways, type I receptors that activate Smad 1, 5 and 8 and those that activate Smad 2 and 3, and each Smad protein complexes with common Smad 4 to enter the nucleus. Specificity of each signal is thought to occur via a number of methods. This includes the level of affinity of TGF- $\beta$ ligands for type I and type II receptors, and Smad complex interactions with different types of transcription factors, which undoubtedly will interact differently with different genes (Dijke and Hill 2004).

\subsubsection{Role of TGF- $\beta$ in reproduction}

There are a number of TGF- $\beta$ superfamily members implicated in the regulation of female reproduction. For example, the TGF- $\beta$ subfamily is involved in local regulation of GC proliferation. Signaling across the hypothalamic-pituitary axis is regulated by inhibin and activin (Bilezikjian, Blount et al. 2004) and anti-Mullerian hormone $(\mathrm{AMH})$ is involved in the regulation of recruiting follicles into the pool of primary and pre-antral follicles (Durlinger, Gruijters et al. 2002; Gruijters, Visser et al. 2003). A brief overview of these subfamilies will be discussed below. However, the primary focus will be on GDF9 and BMP15. They are the only oocyte exclusive TGF- $\beta$ related growth factors identified so far. Their expression pattern, knockout 
(KO) and mutations indicate an important role for these factors in female mammalian fertility. Another family of growth factors identified in oocytes are the fibroblast growth factors (FGF). The best studied is FGF8, which has been implicated in oocyte regulation of glycolysis and steroidogenesis (Sugiura, Su et al. 2007; Miyoshi, Otsuka et al. 2010). However, while the role of FGF members in follicular growth regulation deserves further investigation, it will not be discussed further as it is out of the scope of the present study.

\subsubsection{TGF- $\beta$ subfamily}

The TGF- $\beta$ subfamily consists of three members, TGF- $\beta 1$, TGF- $\beta 2$ and TGF- $\beta 3$. They have been localised to GC, theca cells (TC) and the oocyte by in situ hybridisation and immuno-histochemistry in several different species (Gilchrist, Morrissey et al. 2003; Shimasaki, Moore et al. 2004; Juengel and McNatty 2005). However there are differences in expression localisation and timing between species. While all three members were found in the oocyte, TGF- $\beta 1$ and 3 were found in TC as well, and TGF- $\beta 2$ and 3 were also present in GC (Gilchrist, Morrissey et al. 2003; Juengel, Bibby et al. 2004; Juengel and McNatty 2005). In humans, TGF- $\beta 1$ was found in oocytes of primary follicles and in GC and TC of antral follicles while in rats, TGF- $\beta 1$ was detected in oocytes and GC of pre-antral follicles and in TC from antrum formation (Juengel and McNatty 2005). The cellular origin of TGF- $\beta$ members also differ between species, in some species (rats and humans) they are derived from both TC and GC while in other species (sheep and pigs) they are derived mainly from TC (Juengel and McNatty 2005). 
Signal transduction of TGF- $\beta$ members are achieved via the type II receptor, TGF- $\beta$ receptor II (TGF- $\beta$ RII), and the type I receptor, activin receptor-like kinase 5 (ALK5, also known as TGF- $\beta$ RI). Betaglycan, a co-receptor, is also necessary for binding of TGF- $\beta 2$ (Juengel and McNatty 2005). Expression of ALK5 is found in oocytes of all stages of development in sheep and mice. It was also found in GC of pre-antral follicles, and the stage when expression was first observed ranged from primordial/primary to small pre-antral follicles. The expression of TGF- $\beta$ RII is more variable. Some studies showed expression was in TC of pre-antral and antral follicles while other studies showed that it was in GC of pre-antral and antral follicles (Juengel and McNatty 2005). The authors have suggested that the difference may be due to different techniques used or inherent species differences (Juengel and McNatty 2005). Lastly, betaglycan, which is required by TGF- $\beta 2$ for receptor binding, was found to be expressed in the oocyte, GC and TC of pre-antral and antral follicles in rats and sheep and strong staining reported in TC of human follicles (Drummond, Le et al. 2002; MacConell, Leal et al. 2002; Liu, Kuulasmaa et al. 2003).

The Smads activated by the TGF- $\beta$ subfamily are Smad 2 and 3 (Dijke and Hill 2004). These have been localised in oocytes, GC and TC of follicles at most stages of follicular development (Juengel and McNatty 2005). Taken together, the likely target cells for TGF- $\beta$ subfamily action are GC and TC of pre-antral and antral follicles, since these cells show expression of both TGF- $\beta$ RII and ALK5 receptors and have the appropriate Smad proteins to propagate signal transduction (Juengel and McNatty 2005). 


\subsubsection{Inhibin-Activin system}

Robertson, Foulds et al. first purified inhibin from bovine follicular fluid in 1985. In this and subsequent experiments, inhibin was estimated to be a protein around $56 \mathrm{kDa}$ and composed of two peptide subunits, $\alpha$ and $\beta$, that are linked via disulfide bonds (Miyamoto, Hasegawa et al. 1985; Robertson, Foulds et al. 1985; Fukuda, Miyamoto et al. 1986; Mason, Niall et al. 1986; Robertson, de Vos et al. 1986). To date, the bestcharacterised forms of inhibin are inhibin A and inhibin B. Both forms have an identical $\alpha$ subunit: the inhibin forms are named according to their distinct $\beta$ subunit, and the two forms identified in the ovary are: $\beta_{\mathrm{A}}$ and $\beta_{\mathrm{B}}$ (Mason, Niall et al. 1986).

During purification for inhibin proteins, Ling, Ying et al. (1986) noticed another protein with similar molecular weight but which stimulated FSH release and thus was termed activin. Activin is composed of two inhibin $\beta$ subunits (Bilezikjian, Blount et al. 2006). Since there are different forms of the $\beta$ subunit there are also different forms of activin. The two most prevalent forms involved in follicular growth are activin $A$ and activin $B$. There are also $\beta_{C}, \beta_{D}$ and $\beta_{E}$ forms of activin but as these appear not to be involved in reproduction they will not be discussed further here (Hotten, Neidhardt et al. 1995; Oda, Nishimatsu et al. 1995). As for inhibin, the form of activin is named according to what form of $\beta$ subunit it is composed of, activin $A$ is composed of two $\beta_{\mathrm{A}}$ subunits and activin $\mathrm{B}$ is composed of two $\beta_{\mathrm{B}}$ subunits (Bilezikjian, Blount et al. 2006).

Signal transduction of activin is via either activin receptor type II or type IIB (ActRII/B) followed by oligomerisation with ALK4 (also known as activin receptor type IB, ACVRIB) type I receptor (Bilezikjian, Blount et al. 2006). This activates 
Smad2 and/or Smad3 by phosphorylation and is translocated to the nucleus where they modify target gene expression, such as increasing FSH receptor (FSHR) expression (Bilezikjian, Blount et al. 2006).

The most well known action of activin is in the pituitary gland. Activin acts on two fronts to stimulate FSH secretion (Welt, Sidis et al. 2002). The first is by autocrine/paracrine fashion to stimulate $\mathrm{FSH} \beta$ subunit synthesis (Welt, Sidis et al. 2002; Bilezikjian, Blount et al. 2006). The second is by sensitising the pituitary to GnRH stimulation, which in turn increases FSH secretion (Bilezikjian, Blount et al. 2006).

As a potent promoter of follicular growth, activin is tightly regulated by inhibin and follistatin (Knight 1996; Fraser, Groome et al. 1999; Welt, Smith et al. 2001; Welt, Sidis et al. 2002; Bilezikjian, Blount et al. 2006). The mechanism by which inhibin suppresses FSH is thought to occur via negative endocrine feedback and by interaction with activin in the pituitary (Welt, Sidis et al. 2002; Bilezikjian, Blount et al. 2006). It is thought that inhibin antagonises activin action by competing for binding to ActRII/B. The affinity of inhibin to the receptor can be increased by auxiliary receptors such as betaglycans since by itself it has low affinity to ActRII/B (Welt, Sidis et al. 2002; Bilezikjian, Blount et al. 2006).

Follistatin is composed of an N-terminal segment, two to three follistatin domains and a C-terminal tail (Ueno, Ling et al. 1987; Harrison, Gray et al. 2005). Mutagenesis studies have found the 63-residue N-terminal to be critical for follistatin bioactivity, however it is not sufficient and at least the first two follistatin domains must also be 
present for bioactivity (Keutmann, Schneyer et al. 2004). Substitution experiments of residues indicate that hydrophobic residues are very important for follistatin bioactivity (Sidis, Schneyer et al. 2001; Keutmann, Schneyer et al. 2004). Mutations of tryptophan verses charged residues in the N-terminal suggested that follistatin may hydrophobically interact with activin, and in this way sequesters activin before it has the opportunity to bind to ActRII/B (Sidis, Schneyer et al. 2001; Welt, Sidis et al. 2002).

In the ovary, GC express activin, inhibin and follistatin, and there is evidence for local regulation of follicular growth and oocyte maturation by these proteins (Knight and Glister 2001; Knight and Glister 2003; Gilchrist, Ritter et al. 2004). Activin has been shown in vitro to promote GC proliferation (Miro and Hillier 1996), promote FSHR expression in rat GC (Xiao, Robertson et al. 1992; Nakamura, Minegishi et al. 1993), and promote aromatase activity and oestradiol production in cow GC (Hutchinson, Findlay et al. 1987). These actions suggest that activin plays a role in promoting folliculogenesis. In fact, when activin was over-expressed in vivo, through inactivation of the inhibin $\alpha$ subunit gene, ovarian tumours developed as a consequence of uncontrolled GC proliferation (Matzuk, Finegold et al. 1992). In contrast, in activin type IIB receptor deficient mice, follicular atresia was common and no ovulation was evident (Matzuk, Kumar et al. 1995). However, in this study, the mutant mice also showed reduced levels of FSH. Knight and Glister (2003), have proposed that activin plays an important role in the transition of follicles from the gonadotrophin-independent to the gonadotrophin-dependent stage of folliculogenesis however this remains to be proven. 
As is the case in the pituitary, the actions of activin in the ovary can be antagonised by inhibin and follistatin in developing follicles. Activin produced by GC act to enhance FSH action by increasing FSHR expression in GC, while inhibin enhances LH action. In small pre-ovulatory follicles, activin acts to enhance oestrogen synthesis and increase in FSHR expression in GC (Knight and Glister 2001). This is thought to be due to higher expression of activin in small follicles (Knight and Glister 2001). However, it could also be due to lower expression of follistatin, the activin modulator, in GC at this stage (Knight and Glister 2001). As follicles grow towards ovulation, inhibin and follistatin expression is increased and this results in a shift towards LHR expression and increased androgen production by TC in the dominant follicle (Knight and Glister 2001). This has been shown in vitro by the addition of inhibin A to TC, which showed it promoted androgen synthesis in several different species (Hsueh, Dahl et al. 1987; Hillier and MirÓ 1993; Campbell and Baird 2001).

Many of the affects seen by addition of activin in vitro on GC can be reversed through addition of either inhibin or follistatin (Findlay 1993; Knight and Glister 2003; Glister, Groome et al. 2006). Studies have also found follicles to differentially expresses activin to inhibin as the follicle progresses through folliculogenesis (reviewed in Schwall, Mason et al. 1990). Thus, activin, inhibin and follistatin act in co-ordination with each other during folliculogenesis to influence the nature of FSH and LH signaling from the pituitary as well as the follicular responsiveness to these gonadotrophins. 


\subsubsection{Anti-Mullerian Hormone}

Anti-Mullerian hormone (AMH) has been identified in the regulation of Mullerian duct regression during male sex determination (Durlinger, Gruijters et al. 2001; Gruijters, Visser et al. 2003; Rey, Lukas-Croisier et al. 2003; Visser and Themmen 2005). $\mathrm{AMH}$ is a $140 \mathrm{kDa}$ protein that has bioactivity even before the pro-region is cleaved although bioactivity is greatly enhanced once it is cleaved (Rey, LukasCroisier et al. 2003). Upon cleavage, the $140 \mathrm{kDa}$ protein gives rise to an $110 \mathrm{kDa} \mathrm{N}$ terminal protein, which is thought to enhance the bioactivity of the $25 \mathrm{kDa} \mathrm{C}$-terminal protein (Rey, Lukas-Croisier et al. 2003).

The function of AMH in males is well established. It is expressed most strongly during foetal and pre-pubertal life of the male (Lane and Donahoe 1998). In the foetus, AMH expression causes the regression of Mullerian ducts that would in its absence lead to the differentiation of the vagina, uterus and oviducts (Lane and Donahoe 1998; Durlinger, Kramer et al. 1999). The regulation of AMH expression in males appears to be primarily under the control of SOX9 (Lane and Donahoe 1998).

In females, the function of AMH is less clear (Durlinger, Kramer et al. 1999) notwithstanding the fact that $\mathrm{AMH}$ expression is detectable throughout reproductive life (Gruijters, Visser et al. 2003; Visser and Themmen 2005). Initially, it was thought that $\mathrm{AMH}$ was not important in female fertility because no apparent changes in fertility of AMH KO female mice were observed. However, Durlinger, Kramer et al. (1999) showed that AMH had significant, albeit subtle, effects on fertility. These authors found no differences in the cycle length or differences in the initial size of the primordial pool but by four and 13 months, AMH KO mice had significantly fewer 
primordial but greater numbers of growing follicles than their wild type (WT) counterparts. From these results, Durlinger, Kramer et al. (1999) suggested that AMH was involved in controlling recruitment of primordial follicles into the growing pool. In subsequent studies, Durlinger, Gruijters et al. (2001) found that follicles were less responsive to $\mathrm{FSH}$ when cultured with $\mathrm{AMH}$, and more growing follicles were observed in AMH KO mice, even though the FSH concentration was less. From these results, they hypothesised that $\mathrm{AMH}$ inhibits initial follicle recruitment by controlling FSH sensitivity in follicles (Durlinger, Gruijters et al. 2001).

In females, $\mathrm{AMH}$ expression has been localised to $\mathrm{GC}$ of growing follicles. Its expression is detectable once follicles are recruited from the primordial stage into the growing pool and reduces in atretic follicles and pre-ovulatory follicles (Gruijters, Visser et al. 2003). The type II receptor for AMH is the AMH-specific type II receptor (AMHRII). However, AMHRII mRNA do not seem to be present in GC or oocytes of primordial follicles (McNatty, Reader et al. 2007). AMHRII expression is first found in GC of primary but possibly also later in TC (Gruijters, Visser et al. 2003). The expression pattern of AMH and of its receptor gives some support to Durlinger's hypothesis but further studies are needed as it remains uncertain how AMH promotes the departure of follicles from the primordial pool given that the type II receptor for $\mathrm{AMH}$ is not evident in these follicles.

\subsection{GDF9 and BMP15}

Among members of the TGF- $\beta$ superfamily, so far only two growth factors have oocyte unique gene expression. These are growth differentiation factor 9 (GDF9) and 
bone morphogenetic protein 15 (BMP15) (Dong, Albertini et al. 1996; Laitinen, Vuojolainen et al. 1998; Aaltonen, Laitinen et al. 1999).

\subsubsection{GDF9}

McPherron and Lee first identified GDF9 in 1993. Unlike other previously identified TGF- $\beta$ members, the authors found GDF9 expression was only in mammalian ovaries (McPherron and Lee 1993). However, GDF9 mRNA has also been localised to a number of non-gonadal tissue, such as the hypothalamus, and bone marrow, in rodents and humans (Fitzpatrick, Sindoni et al. 1998). In ovarian tissue, GDF9 mRNA and protein have oocyte-specific expression (Aaltonen, Laitinen et al. 1999; Juengel, Hudson et al. 2002), and expression is evident throughout folliculogenesis from the primordial or primary stages in most species (Juengel and McNatty 2005; Sun, Lei et al. 2010). However a recent study has localised GDF9 protein to GC of both pig and human follicles, although none was found in GC in the mouse (Sun, Lei et al. 2010). The authors of this study did not speculate as to why GDF9 protein was found in pig and human GC nor the significance of this finding. It is possible that GC sequestered GDF9 protein and thus the reason why the immuno-histochemical procedure detected its presence in GC. However, further studies are required to confirm this finding.

Inactivating genetic mutations in GDF9 result in sterility in animals due to abnormal follicular growth. In GDF9 KO mice, ovarian follicular development is compromised at the primordial to primary follicular growth stage and thereafter no normal follicles develop resulting in permanent sterility (Dong, Albertini et al. 1996). Immunisation of sheep against GDF9 also results in this phenotype (Juengel, Hudson et al. 2002). Interestingly, partial immunisation results in increased ovulation rates and there are 
two GDF9 mutations so far identified that also result in increased ovulation rates in the heterozygous animal whereas homozygous animals are sterile. The first was identified by Hanrahan, Gregan et al. (2004) in the Cambridge and Belclare sheep, the other was more recently by Nicol, Bishop et al. (2009) in the Thoka sheep.

GDF9 protein is thought to act as a non-covalent dimer through the type II BMP receptor (BMPRII) and the type I receptor, ALK5 (Vitt, Mazerbourg et al. 2002; Mazerbourg, Klein et al. 2004; Mottershead, Pulkki et al. 2008). The type I receptor kinase phosphorylates Smad2, and/or Smad3 proteins, which together with Smad4 are translocated to the nucleus to influence transcription (Kaivo-Oja, Bondestam et al. 2003; Mazerbourg, Klein et al. 2004; Mottershead, Pulkki et al. 2008).

\subsubsection{BMP15}

BMP15 was identified by a homology search using GDF9 as a template and is also known as GDF9B (Dube, Wang et al. 1998; Laitinen, Vuojolainen et al. 1998; Aaltonen, Laitinen et al. 1999; Jaatinen, Laitinen et al. 1999). The expression pattern of BMP15 was found to be very similar to that of GDF9 being oocyte-specific and evident in oocytes from the primary stage of follicular development (Dube, Wang et al. 1998; Aaltonen, Laitinen et al. 1999; Juengel and McNatty 2005). However, recently BMP15 protein has also been found in GC of pig and human follicles (Sun, Lei et al. 2010). Again further studies are required to establish whether BMP15 mRNA can be localised to GC as the evidence from other laboratories suggest that BMP15 mRNA is not present in GC of sheep, rats, mice or humans (Laitinen, Vuojolainen et al. 1998; Juengel and McNatty 2005; Crawford, Heath et al. 2011). 
Homozygous BMP15 mutations or full immunisation against BMP15 bioactivity in sheep results in complete infertility due to impaired folliculogenesis evident from the primordial to primary stage of growth (Galloway, McNatty et al. 2000; Juengel, Hudson et al. 2002). However, heterozygous BMP15 mutations or partial inhibition of bioactivity results in increased ovulation rates (Galloway, McNatty et al. 2000; Juengel, Hudson et al. 2002). In contrast, in the BMP15 homozygous KO mouse ovarian follicular development and ovulation occur leading to successful pregnancies although litter sizes are slightly reduced (Yan, Wang et al. 2001). Furthermore in mouse, BMP15 does not seem to be critical until just before ovulation and not during the earlier stages of folliculogenesis (Yoshino, McMahon et al. 2006).

These results suggest that oocyte-derived GDF9 and BMP15 have essential roles in folliculogenesis but that their requirements may differ between low and high ovulation-rate phenotypes (Dong, Albertini et al. 1996; Galloway, McNatty et al. 2000; Juengel, Hudson et al. 2002; Di Pasquale, Beck-Peccoz et al. 2004; Hanrahan, Gregan et al. 2004; Nicol, Bishop et al. 2009).

A common feature between these two growth factors is lack of a conserved cysteine residue in the mature protein, which is commonly used for dimerisation (McPherron and Lee 1993; Jaatinen, Laitinen et al. 1999). It is hypothesised that GDF9 and BMP15 may form non-covalently linked homodimers or are able to function has monomers. Another hypothesis is that BMP15 and GDF9 may form non-covalently linked heterodimers due to their closeness in homology (Shimasaki, Moore et al. 2004). This however remains to be proven in vivo. Liao, Moore et al. (2003) and McIntosh, Lun et al. (2008) showed with in vitro experimentation using recombinant 
proteins that heterodimer formation was a possibility. However for this to happen it would occur via the secreted BMP15 pro-region, which was shown to associate with GDF9 mature region during immuno-precipitation experiments (McIntosh, Lun et al. 2008). The evidence from Western immuno-staining experiments with expression systems producing recombinant forms of BMP15 or from experiments with sheep follicular fluid, suggests that BMP15 is secreted either as unprocessed pro-mature forms or as processed pro- and mature monomers whereas GDF9 is expressed mainly as a fully processed mature form capable of behaving as a mature dimer (McNatty, Lawrence et al. 2006; Edwards, Reader et al. 2008; McIntosh, Lun et al. 2008). From cross-linking experiments, there is no evidence to support the notion that recombinant forms of BMP15 were capable of forming dimers (Edwards, Reader et al. 2008).

The signalling pathway of BMP15 is activated after binding to type II and type I receptors and recruitment of the Smad 1, 5, 8 signalling pathway. BMP15 has been shown to bind to the type I receptor, ALK6 (also known as bone morphogenetic protein receptor type IB, BMPRIB), in co-immuno-precipitation experiments (Moore, Otsuka et al. 2003). Moreover BMP15 activity was blocked when the BMPRII ectodomain (ECD) was co-incubated with BMPRII expressing cells (Moore, Otsuka et al. 2003). Collectively, these studies suggest that the type I receptor for BMP15 is ALK6 and the type II receptor is BMPRII. It is known that ALK6 activates Smad1, 5 and 8, thus to further verify that BMP15 does indeed use ALK6, SDS-PAGE of BMP15 stimulated cell lysate with an anti-phospho-Smad1 antiserum, PS1, was conducted (Moore, Otsuka et al. 2003). Consistent with previously observed data, phosphorylation of Smad1 was observed. However, it is possible the above results are not physiologically relevant. BMP15 and GDF9 are co-expressed throughout 
follicular development from the primary stage onwards. Under in vitro conditions recombinant BMP15 and GDF9 when added together act via the BMPRII and Smad 2/3 pathway (Edwards, Reader et al. 2008; Reader, Heath et al. 2011). Furthermore, the addition of a nuclear factor- $\kappa \mathrm{B}(\mathrm{NF}-\kappa \mathrm{B})$ or a $\mathrm{p}-38$ mitogen-activated protein kinase (MAPK) inhibitor inhibited GC proliferation stimulated by recombinant GDF9 and BMP15 in vitro (Moore, Otsuka et al. 2003; Reader, Heath et al. 2011). This indicates that there may be more than one signalling pathway with respect to BMP15 signal transduction.

\subsubsection{Role of GDF9 and BMP15 in reproduction}

From the above work in mutant strains of mice and sheep GDF9 and BMP15 have been identified as important TGF- $\beta$ superfamily members involved in oocyte maturation and ovulation (McNatty, Lawrence et al. 2006; Gilchrist, Lane et al. 2008; Otsuka, McTavish et al. 2011). These studies have shown that GDF9 and BMP15 are essential factors for normal fertility in mammals with a low ovulation-rate phenotype (Davis, McEwan et al. 1992; Galloway, McNatty et al. 2000; Juengel, Hudson et al. 2002; Di Pasquale, Beck-Peccoz et al. 2004). In mammals with a high ovulation-rate phenotype such as the mouse, GDF9, but not BMP15 is essential for ovarian follicular development and fertility (Dong, Albertini et al. 1996; Yan, Wang et al. 2001).

Work with recombinant GDF9 has shown it can mimic some activities of the oocyte in vitro, such as stimulating GC proliferation (Elvin, Clark et al. 1999; Vitt, Hayashi et al. 2000), inducing the CC phenotype in GC (Elvin, Clark et al. 1999; Erickson and Shimasaki 2000), and regulating CC expansion (Gui and Joyce 2005). Similar to work with GDF9, recombinant BMP15 has also been shown to stimulate GC proliferation 
in vitro (Otsuka, Yao et al. 2000). Up regulation of BMP15 mRNA is associated with increased glycolysis and cholesterol biosynthesis in CC (Sugiura, Su et al. 2007; Su, Sugiura et al. 2008 (2007)), and BMP15 is also likely to be involved in CC expansion (Gueripel, Brun et al. 2006). From these studies, GDF9 and BMP15 are considered to be key candidates for factors involved in the regulation of communication between the oocyte and CC (Eppig 2001; Matzuk, Burns et al. 2002; Juengel and McNatty 2005; McNatty, Smith et al. 2005; Gilchrist, Lane et al. 2008). However, their roles in reproduction are far from completely understood (Juengel and McNatty 2005; McNatty, Smith et al. 2005; Gilchrist, Lane et al. 2008).

In women, there have been many mutations associated with infertility however whether they are causative is still uncertain (Table 1A). To date, one BMP15 mutation has been identified with certainty to cause infertility in women (Di Pasquale, BeckPeccoz et al. 2004). In sheep, so far nine inactivating point mutations have been found in GDF9 (three) and BMP15 (six) in Inverdale, Hanna, Belclare, Cambridge, Lacaune, Rasa Aragonesa, and Thoka (Table 1B). The heterozygous GDF9 or BMP15 mutants have increased ovulation rates whereas the homozygous mutants are sterile with streak-like ovaries (Davis, McEwan et al. 1991; Braw-Tal, McNatty et al. 1993; Hanrahan, Gregan et al. 2004; Nicol, Bishop et al. 2009). However more recently, a new GDF9 mutation has been identified in Brazilian Santa Inês sheep, where heterozygous animals are no different to WT animals but the homozygous sheep showed increased ovulation rates (Silva, Castro et al. 2010). Importantly, animals heterozygous for both GDF9 and BMP15 point mutations have greater than additive ovulation rates supporting the notion that both growth factors co-operate at the localised follicular level (Hanrahan, Gregan et al. 2004). Work by (Liao, Moore et al. 
2004) with recombinant BMP15 and GDF9 containing the Inverdale (FecX $)$, Belclare and Cambridge $\left(\mathrm{FecG}^{\mathrm{H}}, \mathrm{FecX}^{\mathrm{B}}\right)$ mutations also support this idea. In mice, GDF9 and BMP15 also show an intimate relationship as demonstrated by the double mutant KO mice generated by (Yan, Wang et al. 2001). Mice lacking both the GDF9 and BMP15 genes showed similar ovarian morphology to that of GDF9 null mutants, however, mice with one functioning GDF9 gene showed more abnormalities than mice with only the BMP15 null mutation (Yan, Wang et al. 2001). Furthermore when recombinant BMP15 and GDF9 were added together in cultures with GC, synergistic effects on ${ }^{3} \mathrm{H}$-thymidine incorporation, steroidogenesis and inhibin production indicate that these growth factors have co-operative effects (McNatty, Juengel et al. 2005a\&b). Moreover recombinant GDF9 and BMP15 are able to interact with one another in vitro (McIntosh, Lun et al. 2008). The culmination of the above results has led to the hypothesis that BMP15 and GDF9 act co-operatively in vivo (Yan, Wang et al. 2001; Hanrahan, Gregan et al. 2004; Su, Wu et al. 2004; McNatty, Smith et al. 2005; McNatty, Juengel et al. 2005a\&b; Gilchrist, Lane et al. 2008; Otsuka, McTavish et al. 2011). 
Table 1A: Mutations in GDF9 and BMP15 genes associated with aberrant ovarian function in women.

\begin{tabular}{|c|c|c|}
\hline GDF9 & BMP15 & Reference \\
\hline K67E & Y235C & (Di Pasquale, Beck-Peccoz et al. 2004) \\
V216M & & (Dixit, Rao et al. 2005) \\
P103S & & (Palmer, Zhao et al. 2006) \\
T121L & & \\
P374L & & \\
R454C & & \\
2 frame & & \\
shift stop & & (Laissue, Christin-Maitre et al. 2006) \\
codons & (Dixit, Rao et al. 2006) \\
S186Y & L148P & \\
& R61W & \\
& R61E & \\
& R76C & \\
& R76H & \\
& A180T & \\
& A180F & \\
& N196K & \\
& R206H & \\
& E211X & \\
T238A & W221R & (Zhao, Qin et al. 2007) \\
\hline
\end{tabular}

The letter shows the amino acid change and the number shows the position. For example, Y235C is an amino acid change at the $235^{\text {th }}$ position from a tyrosine to a cysteine. The letter $\mathrm{X}$ shows a stop codon.

Table 1B: Mutations in GDF9 and BMP15 alleles that cause sterility or increased ovulation rates in homozygous or heterozygous sheep.

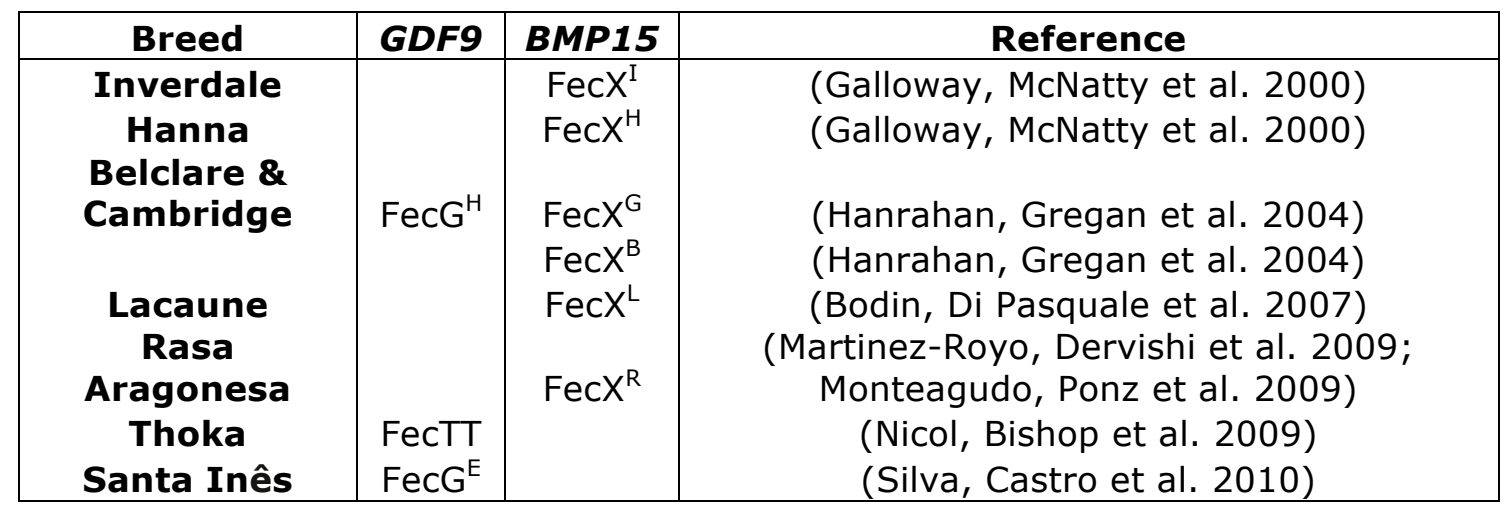

The co-operative effects of GDF9 and BMP15 and the signalling pathway involved are the subject of current research. When these two factors were added to GC in the presence of the BMPRII soluble ECD, ${ }^{3} \mathrm{H}$-thymidine incorporation was inhibited 
(Edwards, Reader et al. 2008; McIntosh, Lun et al. 2008). However, when GDF9 and BMP15 were added to GC in vitro in the presence of a soluble ECD of either ALK 1, 2, 3, 4, 5, 6 or 7, no inhibition of ${ }^{3} \mathrm{H}$-thymidine uptake was noted (Edwards, Reader et al. 2008; McIntosh, Lun et al. 2008). However an inhibitor to ALK 4/5/7 (SB43542) was found to significantly inhibit ${ }^{3} \mathrm{H}$-thymidine uptake (Reader, Heath et al. 2011). These data are interpreted to mean that when BMP15 and GDF9 are added together in vitro they interact with a type II receptor (likely to be BMPRII) to recruit a type 1 receptor (currently not known). The type I receptor is likely to be ALK 4 or 5 since when GDF9 and BMP15 were added together, only the Smad 2 and/or 3 pathway was affected (Reader, Heath et al. 2011).

Notwithstanding many recent studies of the roles of GDF9 and BMP15, our understanding of their functions remains incomplete. Clearly further insights into their physiological and species-specific roles are needed. What several research reports show is that GDF9 and BMP15 act co-operatively both in vivo and in vitro (Yan, Wang et al. 2001; Hanrahan, Gregan et al. 2004; McNatty, Smith et al. 2005; McNatty, Juengel et al. 2005a\&b).

This is a condensed review of GDF9 and BMP15 in female fertility and by no means exhaustive. However, it shows that GDF9 and BMP15 have very important roles in female reproduction. These roles include several aspects of folliculogenesis and oocyte-cumulus cell communication as well as the number of ovulatory follicles which ovulate within a species. Thus, many have hypothesised that GDF9 and/or BMP15 are important factors involved in regulating ovulation quota in animals (McNatty, Juengel et al. 2001; Hanrahan, Gregan et al. 2004; Juengel, Hudson et al. 
2004; Hashimoto, Moore et al. 2005; McNatty, Smith et al. 2005; Shimasaki 2006; McNatty, Hudson et al. 2007).

\subsection{Aims}

The overall aim of this thesis was to test in vitro whether a key difference between a low (sheep) and a high (rat) ovulation-rate species relates, in part, to differences in the role of oocytes and their secretions of GDF9 and BMP15 with respect to GC activity.

To address the overall aims, several research approaches were taken. These included examining: the effects of oocytes from rat or sheep on proliferation of GC from the same species (Chapter 3); whether oocytes from high ovulation-rate species (rat) could stimulate GC proliferation from a low ovulation-rate species (sheep) and vice versa (Chapter 4); the effects of immuno-neutralisation of oocyte-derived GDF9 or BMP15 on ${ }^{3} \mathrm{H}$-thymidine incorporation by GC (Chapter 5); the molecular forms of GDF9 and BMP15 proteins in oocyte lysates and incubation media" (Chapter 6); the effects of time of incubation on GDF9 and/or BMP15 mRNA expression in oocytes" (Chapter 7) and; the effects of time of incubation on gene expression in cumulus cells(Chapter 8).

The key end-points used in these studies were: ${ }^{3} \mathrm{H}$-thymidine uptake by GC in coincubations of GC and oocytes in sheep and rats,; Western blotting to assess the molecular forms of GDF9 and BMP15 proteins in sheep and rat oocyte lysates and incubation media and; QPCR quantification of BMP15 and GDF9 mRNA in sheep and rat oocytes and $\mathrm{CC} / \mathrm{GC}$.. 


\section{Chapter 2 GENERAL METHODOLOGY}

Unless stated below, further details of sources and preparations of reagents are described in Appendix 1. As ovaries were recovered from either the slaughterhouse (lambs) or from routine culling of breeding stocks (rats), ethics approval was not required for the use of animals in the experiments reported in this study. Nevertheless the number of culled rats used was recorded and reported as part of our general animal ethics approvals for rodent research (VUW application number 10695).

\subsection{Ovary collections}

Ovaries from lambs (7-12 months of age) were collected from Taylor Preston Meatworks in Ngauranga, Wellington and transported on ice to the laboratory. Thereafter, at room temperature, the ovaries were trimmed of oviductal tissue, washed in a $3 \%$ bleach-PBS solution $(3 \mathrm{ml}$ of household bleach added to $97 \mathrm{ml}$ of standard PBS stock solution) and then rinsed twice with PBS. The ovaries were kept immersed in the PBS solution during the duration of oocyte collection.

Twenty-one to 28 day-old prepubertal female Sprague-Dawley rats were euthanized by asphyxiation with $\mathrm{CO}_{2}$ gas and ovaries were collected into Leibovitz media (see Appendix 1). On arrival in the laboratory, the ovaries were transferred into a $35 \times 10 \mathrm{~mm}$ Petri dish and excess connective tissue was removed under a dissection microscope. Arteries and veins were cleared of blood by gentle scrapping motion of the dissection scissors. The trimmed ovaries were then placed into a new $35 \times 10 \mathrm{~mm}$ Petri dish with Lebovitz media for oocyte retrieval. 


\subsection{Oocyte and GC collection and preparation}

Sheep cumulus-oocyte-complexes (COC) were collected by puncturing and aspirating all visible antral follicles (approx range of diameters was 2.5-4mm) using a $20 \mathrm{~g}$ needle fitted to a $5 \mathrm{ml}$ syringe. A small amount of Leibovitz media was drawn into the syringe first to prevent oocytes sticking to the syringe wall. After collection of oocytes from three to four ovaries, the needle was removed and the syringe emptied into a $35 \times 10 \mathrm{~mm}$ Petri dish. The aspirated pool was allowed to settle. Using a handdrawn glass pasture pipette, intact and normal-looking COC with at least one layer of cumulus cells (CC) were collected and transferred into a $1 \mathrm{ml}$ microcentrifuge tube containing Leibovitz media. Additionally, granulosa cells (GC) were collected from sheep follicles measuring between $3-4 \mathrm{~mm}$ in diameter. To obtain these, whole ovaries were cut in half using a pair of scissors. Each half was then examined for follicles between $3-4 \mathrm{~mm}$. These follicles were dissected out in a $35 \times 10 \mathrm{~mm}$ Petri dish with no media. An initial small cut to the follicle allowed for the slow release of follicular fluid and then more cuts were made to open the follicle like a flower. A platinum loop was used to scrape GC and the COC from inside the follicular wall. The empty follicular shell was then rinsed with $1 \mathrm{ml}$ Leibovitz to release any remaining GC. The COC was removed and added to the collection of COC in the microcentrifuge tube. Any clumps of GC were dispersed by gentle mixing in Leibovitz with a $20 \mathrm{~g}$ needle fitted to a $3 \mathrm{ml}$ syringe and were transferred into a $15 \mathrm{ml}$ tube.

Sheep follicles that were obviously atretic were discarded. Five criteria were used to determine if a follicle was atretic. The first and second criteria were the presence of lipid debris in the follicular fluid and/or lack of an oocyte with multiple layers of 
compact $\mathrm{CC}$ with a compact centre. These criteria were indicative of a severe compromise in follicular health and these follicles were immediately discarded. The third criteria was based on results from previous studies (McNatty 1981) which report that healthy follicles contain approximately one million $\mathrm{GC} / \mathrm{mm}$ follicular diameter and therefore only follicles with more than 0.5 million $\mathrm{GC} / \mathrm{mm}$ diameter were used. Lastly the colour and degree of vasculature of the theca layer indicate if the follicle had access to blood capillaries and thus nutrients. These criteria are somewhat subjective and if a follicle was on the verge of being accepted or discarded, these characteristics were taken into account in the final decision.

Rat ovaries were placed into a $35 \times 10 \mathrm{~mm}$ Petri dish containing Lebovitz media and all visible follicles were punctured with a $20 \mathrm{~g}$ needle fitted to a $3 \mathrm{ml}$ syringe. The depleted ovaries were then rinsed with Lebovitz media and discarded. Normal-looking COC were removed from the pool by hand-drawn glass pasture pipettes and transferred into a $1 \mathrm{ml}$ microcentrifuge tube. Following collection of all normal COC, the remaining pool of cells was gently mixed and dispersed by collecting into a $3 \mathrm{ml}$ syringe containing Leibovitz media through a $20 \mathrm{~g}$ needle. This step was repeated 2-3 times. Once debris and oocytes had settled to the bottom of the $35 \times 10 \mathrm{~mm}$ Petri dish, GC were aspirated from near the surface, with care taken to avoid debris or COC, using a $20 \mathrm{~g}$ needle and $3 \mathrm{ml}$ syringe and transferred into a $15 \mathrm{ml}$ tube.

Both sheep and rat GC were washed by centrifugation for five minutes at 453 . The supernatant was carefully removed and the GC pellet was re-suspended in $0.5 \mathrm{ml}$ of M199 media (see Appendix 1) and the centrifugation step was repeated. GC were resuspended in a volume of $0.5 \mathrm{ml}$ of M199 media. 
For both species, approximately $250 \mathrm{COC}$ were collected into approximately $5-10 \mu \mathrm{l}$ of Leibovitz media in a $1 \mathrm{ml}$ microcentrifuge tube. The COC were mixed for a total of $2 \mathrm{~min}$ (20sec intervals with $1 \mathrm{sec}$ rests) to strip the oocytes of cumulus cells (denuded oocytes) using a vortex (IKA ${ }^{\circledR}$ MS3 basic vortex). The degree of denudation was checked under the dissecting microscope and subjected to further vortexing (10sec bursts) if required.

Denuded oocytes that were round and undamaged with only a residual number of cumulus cells (i.e. $<<1$ layer) were chosen and included in the bioassays (see Figures 2B and 2D).

\subsection{GC viability test and dilution}

Trypan blue stain was used to assess cell viability. Intact (viable) cells are able to exclude Trypan blue leaving the cell transparent in appearance while dead cells incorporate the dye and appear blue in colour. After the final re-suspension of GC, $10 \mu 1$ was mixed with $10 \mu$ l Trypan blue. The number of dead and live cells was counted using a haemocytometer and cell counter and the numbers of cells in the suspension volumes were calculated as follows:

$\mathrm{N} \times 25 / \mathrm{H} \times 10^{4} \times \mathrm{SV}=$ total number of cells

Key: $\mathrm{N}=$ number of $\mathrm{GC}$ counted

$\mathrm{H}=$ number of haemocytometer cells counted

$\mathrm{SV}=$ suspension volume 
In Chapters 3 and 4, a total of 20,000 GC were used per well in the co-incubation experiments whereas in Chapters 5 and 7, a total of 20,000 live GC were used in the co-incubation experiments with denuded oocytes (DO). The number of GC was calculated and the solution diluted to give $1,000,000$ cells per ml so that each $20 \mu \mathrm{l}$ had 20,000 GC. The number of GC in a suspension used in the following equation depended on whether total or live GC were to be used. The equation below was used:

$\mathrm{N} / \mathrm{R}$ = final volume of suspension $(\mathrm{ml})$

Key: $\mathrm{N}=$ number of $\mathrm{GC}$ in a suspension volume

$\mathrm{R}=$ required number of $\mathrm{GC}$ in $1 \mathrm{ml}$

\subsection{Cell incubation conditions}

For all cell incubation experiments (unless otherwise specified), cell incubations were carried out at $37^{\circ} \mathrm{C}$ and $95 \%$ humidity under $5 \% \mathrm{CO}_{2}$ conditions The incubator used was a shared facility between people and so the temperature was set at $37^{\circ} \mathrm{C}$, although sheep body temperature is normally at $39^{\circ} \mathrm{C}$. For all ${ }^{3} \mathrm{H}$-thymidine uptake (cell proliferation) experiments, the total incubation volume was $55 \mu 1$ prior to the addition of ${ }^{3} \mathrm{H}$-thymidine.

\subsubsection{Bioassays}

All incubations were started on the day of sample collection. Using flat-bottomed 96-

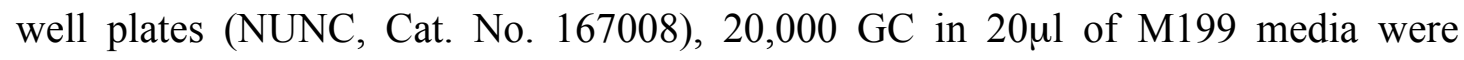
transferred into each treatment well. The DO were washed once in Leibovitz media in a clean 35x10mm Petri dish and then transferred into M199 media. The appropriate numbers of DO in a total volume of $30 \mathrm{ml}$ were transferred into each treatment well. 
Thereafter, the appropriate treatment was added to each well to a final volume of $55 \mu \mathrm{l} /$ well. The plate was labelled, placed in the incubator and the time recorded.

For growth factor neutralisation, specific antibodies to the growth factors, GDF9 or BMP15, were added to each of the treatment wells (see Chapter 5 for antibody details). Stock antibodies were diluted in M199 to suitable concentrations and added in $5 \mu \mathrm{l}$ aliquots to give the required final concentration in $55 \mu \mathrm{l} /$ well. While preliminary antibody titrations were performed to estimate the efficacies of the antibodies to neutralise bioactivities of DO factors stimulating ${ }^{3} \mathrm{H}$-thymidine incorporation, these tests were not exhaustive to fully explore the dose ranges, as only limited supplies of antibodies were available.

The key end-point in the bioassays was to measure GC proliferation in response to coincubations with DO in the presence or absence of specific antibodies to the growth factors GDF9 or BMP15. The amount of ${ }^{3} \mathrm{H}$-thymidine incorporation during DNA synthesis was used as an indirect measure of the level of cell proliferation in each well in response to the different treatments (McNatty, Juengel et al. 2005a, 2005b; Juengel, Reader et al. 2006).

\subsubsection{Western blotting}

Both sheep and rat $\mathrm{COC}$ were incubated based on a previous in-house protocol established by Mr Steve Lawrence at AgResearch, Wallaceville Animal Research Centre, Upper Hutt and Invermay Research Centres. The COC were transferred into 0.2ml PCR tubes and left to settle for a few seconds. Then the Leibovitz media was carefully removed and the COC were washed twice in M199 media. Incubations of 
COC were at a concentration of two COC/ $\mu$ l of M199. Thus, groups of $100 \mathrm{COC}$ were incubated in $50 \mu \mathrm{l}$ of M199. After $24 \mathrm{~h}$ of incubation, the $50 \mu \mathrm{l}$ of spent media were collected into new $0.2 \mathrm{ml}$ PCR tubes, and an aliquot of $50 \mu 1$ of fresh M199 media was added to the COC sample. These will, respectively, become the spent media and the oocyte-lysate samples. Both the spent media and the oocyte-lysate samples contained enzyme inhibitors (Roche, Cat. No. 11697498 001) at a concentration of 1:25 to prevent enzymatic degradation of proteins. These were then stored at $-20^{\circ} \mathrm{C}$ until processing.

\subsection{Methyl- ${ }^{3}$ H-thymidine incorporation assays}

This was based on previously published methods by McNatty, Juengel et al. 2005a, 2005b, Edwards, Reader et al. 2008, and McIntosh, Lun et al. 2008. Briefly the incubations of DO and GC were for $24 \mathrm{~h}$ only because the GC phenotype changes over time as the cells begin to luteinize (Juengel, Reader et al. 2006). Thus, the strategy was to allow the recovered cells to settle over an $18 \mathrm{~h}$ incubation period and thereafter ${ }^{3} \mathrm{H}$-thymidine was added for a six hour interval before the reaction was stopped and the samples harvested to measure radioactive uptake.

For all ${ }^{3} \mathrm{H}$-thymidine incorporation bioassays, a stock solution of methyl- ${ }^{3} \mathrm{H}-$ thymidine (Perkin Elmer, Cat. No. NET355001MC, 1mCi) was diluted 1:24 in M199 media. After 18 hours of incubation, $10 \mu$ of diluted methyl- ${ }^{3} \mathrm{H}$-thymidine was added to each well in the 96-well plates and incubated for a further six hours.

A cell harvester (TOMTEC, mod. No. MACH 3-FM series) was used to recover the cells from the 96-well plates. Cells were recovered onto a filter mat (Perkin Elmer 
glass fibre filter mats, 90x120mm, printed Filtermat A, Cat. No. 1450-421), which was then dried and placed into a sample bag (Perkin Elmer sample bag, 90x120mm, Cat. No. 1450-432) containing $5 \mathrm{ml}$ of scintillation fluid (Beta Scint, Perkin Elmer, Cat. No. SC/9200.21). The bag was then sealed with a heat sealer (Wallac, mod. No. 1295-012). The amount of ${ }^{3} \mathrm{H}$-thymidine incorporated by the GC was measured by a beta counter (Perkin Elmer, 1450-SLC MicroBeta TriLux). The beta counter was programmed to count ${ }^{3} \mathrm{H}$ and normalise from 2 minutes.

\subsection{Western Blotting}

Polyacrylamide gels were made according to the protocols listed in Table 2A. The separating gel was made and left to polymerise with a thin layer of water on top. After polymerisation was complete, it was rinsed with distilled water and gently dried.

The stacking gel was prepared and after a comb was inserted, it was left to polymerise. Once polymerisation was complete, the comb was recovered and the plates removed from the stand. The gels were usually made a day before the experiment and stored at $4^{\circ} \mathrm{C}$ in a plastic bag with some distilled water until use.

Table 2A: Table of reagents for $15 \%$ polyacrylamide gels

\begin{tabular}{|c|c|c|}
\hline & 15\% separating gel & $\mathbf{4 \%}$ stacking gel \\
\hline Distilled water & $2.35 \mathrm{ml}$ & $3.0 \mathrm{ml}$ \\
\hline $\mathbf{1 . 5 M}$ Tris-HCl pH8.8 & $2.5 \mathrm{ml}$ & $\begin{array}{c}1.25 \mathrm{ml}(0.5 \mathrm{M} \text { Tris- } \mathrm{HCl} \\
\text { pH6.8) }\end{array}$ \\
\hline $\mathbf{1 0 \%}$ (w/v) SDS & $0.1 \mathrm{ml}$ & $50 \mathrm{ml}$ \\
\hline Bis-acrylamide mix (30\%) & $5.0 \mathrm{ml}$ & $0.65 \mathrm{ml}$ \\
\hline $\begin{array}{c}\mathbf{1 0 \%} \text { (w/v) ammonium } \\
\text { persulphate }\end{array}$ & $50 \mathrm{ml}$ & $25 \mathrm{ml}$ \\
\hline TEMED & $5 \mathrm{ml}$ & $5 \mathrm{ml}$ \\
\hline
\end{tabular}

All samples including the molecular ladder (BioRad, Cat. No. 161-0305) were prepared so their final volume in the gel was approximately the same. Western blot 
sample buffer (see Appendix 1) was added to all samples. The samples were heated to $90^{\circ} \mathrm{C}$ for 5 minutes followed by a quick centrifuge. The pre-made gel was removed from storage and a running cassette was set up and filled with the SDS-PAGE running buffer (see Appendix 1). The gel underwent electrophoresis for an hour at $150 \mathrm{~V}$ at room temperature.

Two scotch pads, a nitrocellulose membrane and two pieces of blotting paper were soaked in Tris-glycine transfer buffer (see Appendix 1). Following gel electrophoresis, a transfer sandwich was made using a transfer sandwich maker. The stacking order was as follows:

Black face (negative charge)

Scotch pads

Blotting paper

Gel

Nitrocellulose membrane

Blotting paper

Scotch pads

Red face (positive charge)

After removing the glass plates from the running cassette, the top glass plate was removed with a scraper, exposing the gel. The stacking gel was cut and removed. A piece of blotting paper was placed on the gel and any air bubbles were gently removed with a roller. Together, they were flipped on to the awaiting scotch pad. Separation of the gel from the glass plate was carefully carried out using a scraper so as not to tear the gel. Each piece was then stacked on top following the stacking order detailed above. Between each layer, transfer buffer was added gently onto the surface to prevent drying and any air bubbles were removed. 
The finished sandwich was placed into the transfer stand and filled with transfer buffer. An ice pack and magnetic stirrer bar was put in with the stand and the transfer occurred at a temperature of $4^{\circ} \mathrm{C}$ for either 1 hour at $100 \mathrm{~V}$ or overnight at $30 \mathrm{~V}$.

To check the transfer success, the bands were visualised with Ponceau S stain (see Appendix 1). The nitrocellulose membrane was stained with Ponceau $\mathrm{S}$ for a minute, then rinsed with distilled water to de-stain. Molecular ladder bands were marked with pencil and the membrane fully de-stained with more distilled water.

The nitrocellulose membrane was blocked with 5\% blotto for an hour with agitation at room temperature. In general, the stock primary antibody was diluted to $1 \mu \mathrm{g} / \mathrm{ml}$ in $5 \%$ blotto. The blocked membrane was then incubated with agitation using a Labnet ORBIT LS at 60 rpm (Total Lab Systems Ltd, Cat. No. S 2030-L S-230V). Incubation was with the primary antibody was carried out overnight at room temperature. If more than one primary antibody was used, the membrane was cut and each section incubated separately with its respective antibodies.

After primary antibody incubation, the membrane was washed 3x 5 minutes with Tris buffer (see Appendix 1). In general, a rabbit anti-mouse-HRP (Abacus, Cat. No. 315035-045) at a concentration of $1 \mu \mathrm{l}: 3000 \mu \mathrm{l}$ in $5 \%$ blotto was used as the secondary antibody. Secondary antibody incubations were carried out at room temperature for 3 hours with agitation. The membranes were then washed $3 \times 5$ minutes with Tris buffer. Freshly prepared Electrical Chemi-luminescence (ECL) reagents (made in-house) were poured over the nitrocellulose membrane and incubated for one minute at room 
temperature. The membrane was then removed, lightly blotted, wrapped with clearwrap and taped down onto an exposure cassette.

X-ray films were exposed and the top left corner cut for orientation prior to development. Once the film was dry, it was aligned with the membrane and the ladder positions were marked.

\subsection{Collection of COC or DO for the time series studies}

Groups of ten rat or sheep COC were collected into $0.2 \mathrm{ml} \mathrm{PCR} \mathrm{tubes} \mathrm{and} \mathrm{washed}$ twice in sterile PBS. After removal of PBS, samples were resuspended in 5 $\mu 1$ of M199 media containing $10 \mu \mathrm{M}$ of Milrinone (a type III phosphodiesterase inhibitor) and incubated for $0,8,16$ or 24 hours in the cell-culture incubator. At each of the specified time points, the appropriate samples were removed from the incubator and immediately stored in a $-80^{\circ} \mathrm{C}$ freezer until RNA extraction and cDNA synthesis. Each individual experiment had three replicates for each time point.

Co-incubations of DO and GC were undertaken as described above for the bioassays.

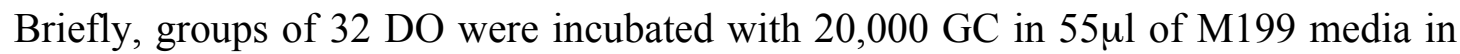
96-well plates. Each individual experiment had two replicate wells per time point. At 0,8 and 24 hours after incubation, samples were removed and stored at $-80^{\circ} \mathrm{C}$ until RNA extraction. Each time point was incubated in separate 96-well plates and the whole plate was removed and frozen. 


\subsection{RNA extraction and cDNA synthesis}

The ArrayPure Nanoscale RNA purification kit (Epicentre Biotechnologies, Cat. No. MPS04050) was used to extract total RNA. The TE buffer used to reconstitute the precipitated total RNA was made in-house (see Appendix 1), and all other specialised reagents were supplied in the kit.

In brief, the total RNA purification procedure involved adding 30 4 l of Nano-scale Lysis Solution plus $1 \mu 1$ Proteinase $\mathrm{K}$ into each sample and transferring into a clean microcentrifuge tube. The contents were mixed briefly by vortexing followed by 15 minutes incubation at $65^{\circ} \mathrm{C}$. Samples were placed on ice for five minutes followed by addition of $18 \mu \mathrm{l}$ of MPC Protein Precipitation Reagent into each tube and then vortexed briefly. Tubes were then centrifuged for seven minutes at 13,000 at $4^{\circ} \mathrm{C}$. The supernatant was transferred into a clean microcentrifuge tube and total RNA was precipitated by addition of $50 \mu$ l of isopropanol, vortexed briefly and pelleted by centrifugation at $4^{\circ} \mathrm{C}$ at 12,000 for five minutes. Following aspiration of isopropanol and air-drying of the pellet for five minutes, contaminating genomic DNA was removed by the addition of $40 \mu \mathrm{l}$ of $1 \mathrm{x}$ DNase Buffer plus $1 \mu \mathrm{l}$ of RNase-Free DNase into each tube. Tubes were incubated in a $37^{\circ} \mathrm{C}$ water-bath for 30 minutes. Thereafter, $20 \mu 1$ of 2x Nano-scale Lysis Solution was added and tubes vortexed briefly followed by a further addition of $20 \mu l$ of MPC Protein Precipitation Reagent. The samples were vortexed briefly before being placed on ice for five minutes. All tubes were centrifuged for five minutes at $4^{\circ} \mathrm{C}$ at 12,000 . The supernatant was transferred into a clean microcentrifuge tube and purified total RNA was precipitated with the addition of $50 \mu \mathrm{l}$ of isopropanol. Tubes were briefly vortexed and centrifuged for five minutes at $4^{\circ} \mathrm{C}$ at 12,000 . The isopropanol was aspirated and the remaining pellet washed 
with $70 \%$ ethanol. The samples were centrifuged at 12,000 for $3 \mathrm{~min}$ and the ethanol was aspirated. The pellet containing the purified total RNA was allowed to air dry for five minutes before being re-suspended in $5 \mu 1$ TE buffer.

For the synthesis of cDNA the SuperScript ${ }^{\circledR}$ Vilo ${ }^{T M}$ cDNA Synthesis Kit from Invitrogen (Cat. No. 11754-050) was used. To each sample, $4 \mu$ of 5x Vilo reaction mix, $2 \mu 1$ of $10 x$ SuperScript ${ }^{\circledR}$ Vilo ${ }^{\mathrm{TM}}$ enzyme and $9 \mu 1$ of UltraPure water (Invitrogen; Cat. No. 10977 015) was added to make a final volume of $20 \mu 1$. For the negative RTPCR controls, the enzyme was replaced with $2 \mu$ of UltraPure water. All tubes were well mixed, and RT-PCR was carried out using the Corbett real time PCR machine (Corbett Life Science, mod. No. RG6000). Samples were incubated for 10mins at $25^{\circ} \mathrm{C}, 60 \mathrm{mins}$ at $42^{\circ} \mathrm{C}$ and $5 \mathrm{mins}$ at $85^{\circ} \mathrm{C}$ and were removed immediately following the incubation procedure and stored at $-20^{\circ} \mathrm{C}$ until use.

\subsection{Real time quantitative PCR (QPCR)}

Forward and reverse primers were designed with Beacon Designer version 7.5 (Table 2B and C) based on sequences obtained through NCBI. All primers were ordered from Invitrogen. QPCR can be used to measure mRNA expression of a single gene (singleplex) or multiple genes (multiplex) within one reaction mix. A singleplex reaction requires the presence of a forward and reverse primer using SYBR green technology (pre-made Brilliant II SYBR ${ }^{\circledR}$ Green QPCR Master Mix purchased from Agilent Technologies, Cat. No. 600828), while multiplex QPCR requires forward and reverse primers as well as spectrally different dual-labelled fluorescent probes (Taqman probe, ordered from Sigma-Aldrich) (pre-made Brilliant ${ }^{\circledR}$ Multiplex QPCR Master Mix purchased from Agilent Technologies, Cat. No. 600553). 
Table 2B and C: Forward and reverse primer sequences for rat (B) and sheep (C) QPCR.

B)

\begin{tabular}{|c|c|c|}
\hline & \multicolumn{2}{|c|}{ Rat } \\
\hline Gene & Forward & Reverse \\
\hline Fshr & GCAGAAAGAAAGTCGGTGAATGG & GGGGAGGTCGGTCGGAATC \\
\hline Kitl & TTTGTAATGTGTCCAGTTTGATTCTTG & TCTTGCTATGGGCTTGCTTCC \\
\hline Cx43 & AGACTGCGGATCTCCAAAATACG & CTTGTTGTAATTGCGGCACGAG \\
\hline Arom & CCACTCAAGGGCAAGATGATAAGG & GATGTTAGCACGCAAAGCAGTAG \\
\hline Cycd2 & ACCGATGTGGATTGTCTCAAAGC & GCCGTTGTGCTGCTCTTGAC \\
\hline Rpl19 & GACCCCAATGAAACCAACGAAATC & GGAATGGACAGTCACAGGCTTC \\
\hline Lhr & CСATTGACACTCTGCTTAACTTTC & CTGAGATAGACGAGGAACAAGATG \\
\hline Gdf9 & GGAACACTTACTCAAATCGGTCTTG & GCCTTGCTGGAAGACATGGG \\
\hline Bmp15 & AACGAGGTAGCATACCAACTAATCAG & GTTCGACATTTAGGGACCCAAGG \\
\hline
\end{tabular}

C)

\begin{tabular}{|c|c|c|}
\hline & \multicolumn{2}{|c|}{ Sheep } \\
\hline Gene & Forward & Reverse \\
\hline FSHR & AGGACAGCAAGGTGACAGAGATG & GTAGTTTGGGCAGGTTGGAGAAC \\
\hline KITL & CTGCTGGTGGGCGGGAAG & ATTGCGGGTGTTCTCGTTCTTG \\
\hline CX43 & TCTTCAAGTCTGTCTTCGAGGTG & CTGATGCGGGCAGGGATC \\
\hline AROM & ATCCCGATTTGGCAGTAAACTTGG & GATGGAATCAGCACAGATGGTCAC \\
\hline CYCD2 & GATGCTGGAGGTCTGTGAGGAG & CAGCGGTCAGCGGGATGG \\
\hline RPL19 & TAAGCGAAAGGGTACTGCCAATG & TTCTTAGATTCACGGTATCGTCTGAG \\
\hline LHR & AGGGAATGGGTGTAGTGTTGCTG & TGTGATGACTGTGAGGGTGTAGAC \\
\hline GDF9 & ATTAGCCTTGATTCTCTGCCTTCTAG & GTGTCTCCCACCTAAATGGTTCAG \\
\hline BMP15 & AACCTTGTCAGTGAGCTGGTG & AGATACTCCСАTTTGССTCAATCAG \\
\hline
\end{tabular}

Table 2D and E: Probe sequences for used in rat (D) and sheep (E) multiplex QPCR.

D)

\begin{tabular}{|c|l|}
\hline Gene & \multicolumn{1}{|c|}{ Rat } \\
\hline Rpl19 & [Cy5]CCAATGCCAACTCTCGTCAACAGATCAGGAAG[BHQ3] \\
Gdf9 & [6FAM]CACTCTGAACAACTCCGCTGCTTCCTCCT[BHQ1] \\
Bmp15 & [HEX]CACTGTGGTCTACCGCCATCAACTTCATCTAGT[BHQ1] \\
\hline
\end{tabular}

E)

\begin{tabular}{|c|c|}
\hline Gene & \multicolumn{1}{|c|}{ Sheep } \\
\hline RPL19 & [Cy5]TTCTCATCCTCCTCATCCACGTTACCTTCTCGG[BHQ3] \\
GDF9 & [6FAM]AGTCTCAGCCTCAGATTCCAACGCAGTCCTA[BHQ1] \\
BMP15 & [HEX]AGAATGTCCCTCAGCCTTCCTGTGTCCCT[BHQ1] \\
\hline
\end{tabular}

Optimal primer concentrations were obtained by titrating concentrations of $50 \mathrm{nM}$ to $300 \mathrm{nM}$ of forward primers and reverse primers. The optimal concentration was 
deemed to be the set of primers with the concentration that gave the lowest cycle threshold $\left(\mathrm{C}_{\mathrm{T}}\right)$ value. The optimal primer concentrations are shown in Table $2 \mathrm{~F}$ and $\mathrm{G}$ for singleplex and multiplex QPCR respectively.

Table 2F and G: Forward and reverse primer concentration in $\mathrm{nM}$ for rat and sheep singleplex (F) and multiplex QPCR (G).

F)

\begin{tabular}{|c|c|c|c|c|}
\cline { 2 - 5 } \multicolumn{1}{c|}{} & \multicolumn{2}{c|}{ Rat } & \multicolumn{2}{c|}{ Sheep } \\
\hline Gene & Forward & Reverse & Forward & Reverse \\
\hline FSHR & 200 & 200 & 100 & 100 \\
KITL & 300 & 200 & 200 & 100 \\
CX43 & 100 & 100 & 200 & 50 \\
AROM & - & - & 100 & 200 \\
LHR & - & - & - & - \\
CYCD2 & 200 & 200 & 200 & 100 \\
RPL19 & 300 & 200 & 200 & 200 \\
\hline
\end{tabular}

G)

\begin{tabular}{|c|c|c|c|c|c|c|}
\cline { 2 - 7 } \multicolumn{1}{c|}{} & \multicolumn{3}{c|}{ Rat } & \multicolumn{3}{c|}{ Sheep } \\
\hline Gene & Forward & Reverse & Probe & Forward & Reverse & Probe \\
\hline GDF9 & 200 & 100 & 100 & 200 & 200 & 50 \\
BMP15 & 300 & 200 & 100 & 200 & 200 & 50 \\
RPL19 & 300 & 200 & 100 & 200 & 200 & 50 \\
\hline
\end{tabular}

Once the conditions for the QPCR reactions were optimised, the amplification efficiency for each gene was tested. A cDNA sample was made by halving the sample concentration with each subsequent dilution in UltraPure water. In most cases the dilution series was from 1 to $1: 128$. The reaction efficiency for each gene is listed in Table $2 \mathrm{H}$. 
Table 2H: Table of QPCR efficiency for each candidate genes.

\begin{tabular}{|c|c|c|}
\cline { 2 - 3 } \multicolumn{1}{c|}{} & \multicolumn{2}{c|}{ Efficiency (\%) } \\
\hline Gene & Rat & Sheep \\
\hline FSHR & 92 & 83 \\
KITL & 90 & 89 \\
CX43 & 93 & 90 \\
AROM & 95 & 90 \\
LHR & 90 & - \\
CYCD2 & 92 & 91 \\
RPL19 & 99 & 93 \\
GDF9 & 99 & 96 \\
BMP15 & 99 & 93 \\
\hline
\end{tabular}

The Corbett Rotor-Gene 6000 Series Software 1.7 was used to analyze all QPCR results. The optimal amount of cDNA template per QPCR reaction was deemed to be that that resulted in a $C_{T}$ value within the measureable range (15-26) therefore the cDNA samples were diluted accordingly. Information regarding validation of the multiplex reaction for measuring , and mRNA expression levels including probe optimisation and the primer concentration for was provided by Dr Janet Crawford, Reproduction Group, SBS, VUW.

For each QPCR reaction, a master mix was prepared and dispensed into individual tubes in duplicate. The appropriate amount of sample template (see Table 2I) was then added. Thus the master mix for one sample contained 26ul of either Multiplex or SYBR Green pre-made mixes, forward and reverse primers, and probes if a multiplex reaction was performed. The final volume for a one sample master mix was $52 \mu l$; any remaining difference was made up by UltraPure water in the master mix. Aliquots of $25 \mu 1$ were pipetted in duplicate into $0.1 \mathrm{ml}$ strip tubes (Corbett Life Science, Cat. No.

\footnotetext{
${ }^{1}$ The nomenclature for rat genes are in italics with the first letter capitalised. For sheep genes, they are in italics with all letters capitalised. In cases where both species are referred to, the sheep nomenclature is used.
} 
3001-002). The incubation condition of the samples in the Corbett machine (Corbett Rotor-Gene 6000) was for $10 \mathrm{mins}$ at $95^{\circ} \mathrm{C}$ followed by 40 repeats of $15 \mathrm{sec}$ at $95^{\circ} \mathrm{C}$ and $60 \mathrm{sec}$ at $60^{\circ} \mathrm{C}$. When SYBR Green technology was used an additional melt step was added. This step involved incubating the QPCR products for $5 \mathrm{sec}$ at each $5^{\circ} \mathrm{C}$ interval from $60-95^{\circ} \mathrm{C}$.

Each QPCR reaction had one to four technical replicates performed to test for its repeatability. A calibrator sample was included in each experiment to adjust for reaction variations such as sample volume, different batches of master mixes and dilutions of probes and primers.

Table 2I: Amount, in $\mu \mathrm{l}$, of cDNA for a one sample master mix for rat and sheep singlplex and multiplex QPCR. The 20 $\mu$ l of synthesised cDNA were diluted according to this table. The amount of cDNA for a one sample master mix was taken from the diluted samples.

\begin{tabular}{|c|c|c|c|c|}
\cline { 2 - 5 } \multicolumn{1}{c|}{} & \multicolumn{2}{c|}{ Rat } & \multicolumn{2}{c|}{ Sheep } \\
\hline Gene & Amount (mI) & Dilution & Amount (mI) & Dilution \\
\hline FSHR & 1.04 & $1: 5$ & 1.04 & $1: 5$ \\
KITL & 10.4 & $1: 5$ & 2.08 & $1: 5$ \\
CX43 & 1.04 & $1: 40$ & 1.04 & $1: 40$ \\
AROM & - & - & 2.08 & $1: 5$ \\
LHR & - & - & - & - \\
CYCD2 & 1.04 & $1: 40$ & 1.04 & $1: 40$ \\
RPL19 & 1.04 & $1: 40$ & 1.04 & $1: 5$ \\
Multiplex & 5.2 & $1: 5$ & 2.5 & \\
\hline
\end{tabular}

\subsection{RPL19 mRNA in oocytes}

The ribosomal protein L19 ( $\quad$ ) gene used in this study is a commonly used housekeeper gene (Eppig, Wigglesworth et al. 1997; Sugiura, Pendola et al. 2005; Gilchrist, Ritter et al. 2006; Sugiura, Su et al. 2007; Su, Sugiura et al. 2008 (2007)). As this study involved the collection of COC, it was necessary to check the amount of gene expression in the DO compared to that in the CC. This was particularly important as those genes specific to the $\mathrm{CC}$ were normalised to expression (i.e. 
RNA input) whilst those genes specific to the oocyte were not normalised against expression and were expressed as total mRNA levels relative only to the calibrator sample. Thus, a validation experiment was performed to check the relative levels of mRNA in the oocytes verses levels of mRNA in the CC/GC compartment of a COC.

Sheep COC were collected as described in section 2.2. Initial transfer of the COC into the 96-well plate was by hand-drawn glass pasture pipettes, thereafter COC were transferred carefully by sterile pipetting in a minimal volume $(\sim 2 \mu 1)$ to avoid crosscontamination. Each COC was transferred into a wash well containing $100 \mu$ l of sterile PBS and then into a denudation well containing $10 \mu$ of PBS. Thereafter CC were stripped from the oocytes by vigorous pipetting. Each DO was then transferred through three additional wash wells containing $10 \mu \mathrm{l}$ of PBS, before being placed into a clean well containing $5 \mu 1$ of sterile PBS. A photo was taken of the COC before the denudation step and of the denuded oocyte to record the success of the denudation procedure and any damage that may be caused by this process to the oocyte. Total RNA was extracted and cDNA was synthesised from these samples as described in section 2.2.8. The mRNA expression levels of were measured by the same multiplex reaction as detailed in section 2.2.9 where, , and mRNA expression levels were investigated. The mRNA expression levels of in rat oocytes were provided with permission from Dr Janet Crawford, Reproduction Group, SBS, VUW.

The fold difference between expression levels of in $\mathrm{CC}$ and $\mathrm{DO}$ was calculated

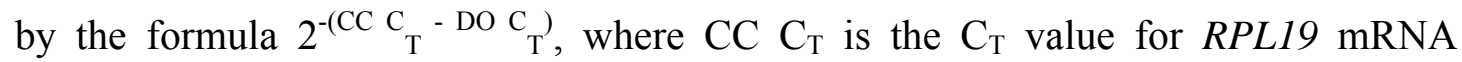


expression in $\mathrm{CC}$ samples and $\mathrm{DO} \mathrm{C}_{\mathrm{T}}$ is the $\mathrm{C}_{\mathrm{T}}$ value for mRNA expression in DO samples. For example, the fold difference between CC and DO of rat sample 1 would be: $2^{-(25.76-30.44)}=25.63(2$ d.p $)$.

In sheep, $\quad$ mRNA levels in DO were $\sim 38.32$ fold less abundant than mRNA expression levels in CC. In the rat mRNA levels in DO were $\sim 110.28$ fold less abundant in the DO than CC.

Table 2J: Cycle threshold $\left(\mathrm{C}_{\mathrm{T}}\right)$ values for RPL19 mRNA in CC and DO and their fold differences in rats and sheep.

\begin{tabular}{|c|c|c|c|c|c|c|}
\cline { 2 - 7 } \multicolumn{1}{c|}{} & \multicolumn{4}{c|}{ Rat } & \multicolumn{2}{c|}{ Sheep } \\
\hline Sample & CC & DO & Fold difference & CC & DO & Fold difference \\
\hline 1 & 25.76 & 30.44 & 25.63 & 22.54 & 26.39 & 14.42 \\
2 & 26.79 & 31.92 & 35.02 & 21.61 & 26.67 & 33.36 \\
3 & 23.22 & 31.60 & 333.14 & 19.29 & 25.96 & 101.83 \\
4 & 23.26 & 31.61 & 326.29 & 21.08 & 24.80 & 13.18 \\
5 & 24.35 & 28.88 & 23.10 & 21.20 & 25.95 & 26.91 \\
6 & 26.86 & 28.43 & $2.97^{*}$ & 19.97 & 25.30 & 40.22 \\
7 & 24.32 & 29.01 & 25.81 & & & \\
\hline
\end{tabular}

The results show that levels of mRNA expression were far less in the DO than in the CC. There were two main reasons where the fold difference between the $\mathrm{CC}$ and the DO were low. The first reason was incomplete removal of $\mathrm{CC}$ from the oocytes, as indicated by the photographic evidence (see Figure 2A and B). The second reason was the use of COC with few CC initially attached (asterisked in Table 2J, see Figure 2C and D). Therefore caution was exercised in all future experiments and only large COC with multiple layers of CC attached to the oocyte was collected.

In conclusion, the results showed that the oocyte did not contribute significantly to the total amount of mRNA expression in COC and therefore could be used to normalise CC-specific candidate genes against in samples of COC. 

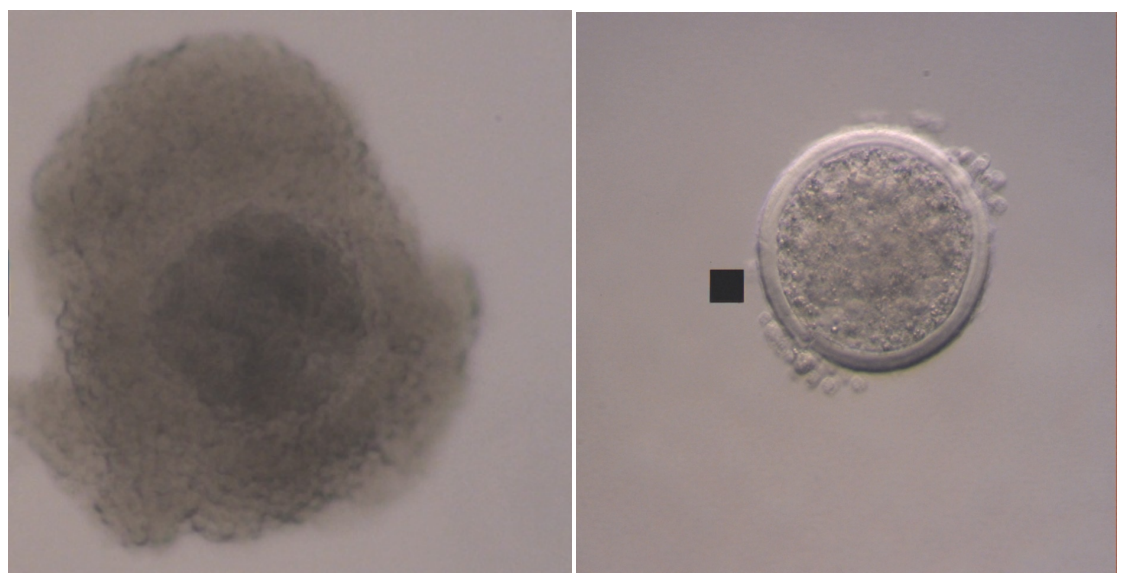

Figure 2A and B: Photographs of the same sheep COC before (A) and after denuding (B) as seen under a high-powered inverted compound microscope. In Figure B, CC still attached to the oocyte can be seen.
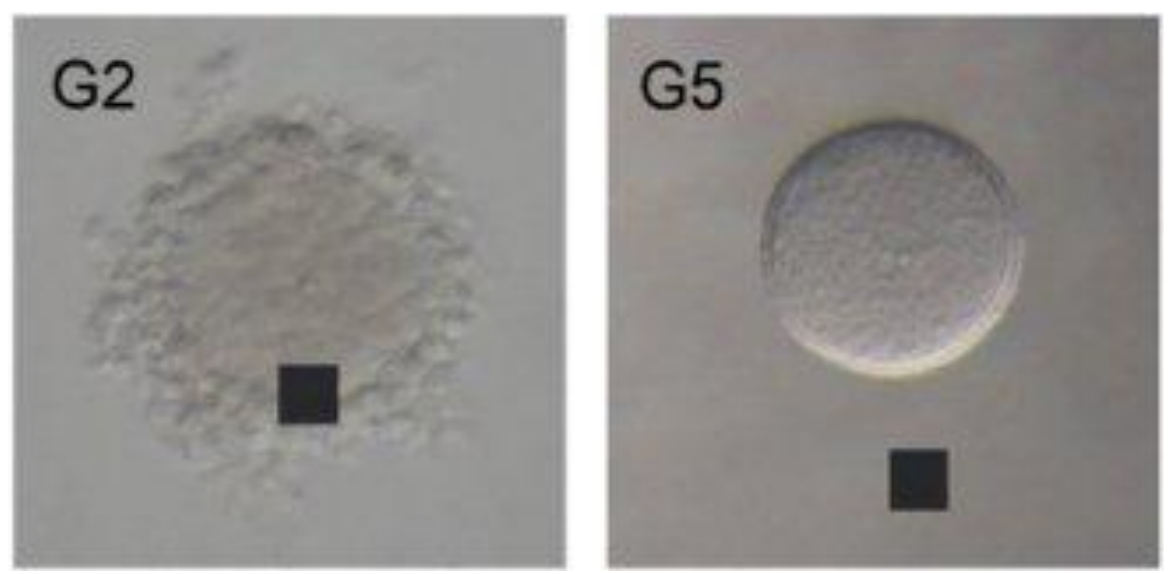

Figure 2C and D: Photographs of the same rat COC before (C) and after denuding (D) as seen under a high-powered inverted compound microscope. Although Figure 2D shows the oocyte has been fully stripped of $\mathrm{CC}$, the starting $\mathrm{COC}$ did not have a large amount of $\mathrm{CC}$ attached. Thus resulting in a small difference in $R P L 19 \mathrm{mRNA}$ levels between the COC and $\mathrm{CC}$. The black squares are a fixed camera setting and are not related to any point of interest in the images. Images supplied by Dr J. Crawford.

\subsection{QPCR Analysis}

For each time series experiment, relative levels of mRNA were calculated using the $2^{-}$ ${ }_{\mathrm{T}}^{\Delta \Delta \mathrm{C}}$ method as described by Livak and Schmittgen (2001). For each CC-specific gene, the $\mathrm{C}_{\mathrm{T}}$ value for expression for each sample was subtracted from the $\mathrm{C}_{\mathrm{T}}$ value for the gene of interest resulting in a $\Delta \mathrm{C}_{\mathrm{T}}$ value. Then the mean $\mathrm{C}_{\mathrm{T}}$ value of the control samples (control wells at Time 0) was subtracted from the $\Delta \mathrm{C}_{\mathrm{T}}$ values 
resulting in a $\Delta \Delta \mathrm{C}_{\mathrm{T}}$ value. To convert these values to linear values (i.e. fold-change) for analyses, the following equation was performed: $2^{-\left(\Delta \Delta C_{T}\right)}$.

For the oocyte-derived genes, the $\mathrm{C}_{\mathrm{T}}$ value for for the calibrator sample was subtracted from the $\mathrm{C}_{\mathrm{T}}$ value for either

or gene expression for each sample resulting in a $\Delta \mathrm{C}_{\mathrm{T}}$ value. Then the mean $\mathrm{C}_{\mathrm{T}}$ value of the control samples (control wells at Time 0) was subtracted from the $\Delta \mathrm{C}_{\mathrm{T}}$ values resulting in a $\Delta \Delta \mathrm{C}_{\mathrm{T}}$ value. To convert these values to linear values (i.e. fold-change) for analyses, the following equation was performed: $2^{-\left(\Delta \Delta C_{T}\right)}$.

Following Livak and Schmittgen (2001), the upper and lower errors were calculated by the formula $2^{-\left(\mathrm{ddC}_{\mathrm{T}}{ }^{ \pm S t d e v}\right)}$, where Stdev stands for standard deviation.

The ratio of mRNA expression between genes was calculated using the formula, $2^{-(1 s t}$ gene $\mathrm{C}_{\mathrm{T}}-2$ nd gene $\left.\mathrm{C}_{\mathrm{T}}\right)$ resulting in a relative fold difference. Correlation coefficients $\left(\mathrm{R}^{2}\right.$ value) were calculated from the generation of the line of best-fit following linear regression of two genes of interest. Residual values were used to check if the linear regression model was valid and to identify outliers. Residual values were calculated by subtracting the $\mathrm{Y}$-axis gene from the predicted $\mathrm{Y}$-axis gene, as calculated by the linear equation. Identified outliers were removed from the final correlation $\mathrm{R}^{2}$ value. An $\mathrm{R}^{2}$ range between 0.6-1.0 indicates a strong correlation, 0.4-0.5 a moderate correlation and 0-0.3 little or no correlation. 


\section{Chapter 3 EFFECTS OF OOCYTE CO-INCUBATION WITH GC ON GC PROLIFERATION IN RAT AND SHEEP}

\subsection{Introduction}

The ability of oocytes to stimulate GC proliferation, as assessed by ${ }^{3} \mathrm{H}$-thymidine incorporation has been demonstrated in mice and cows (Vanderhyden, Caron et al. 1990; Vanderhyden, Telfer et al. 1992; Li, Norman et al. 2000; Gilchrist, Ritter et al. 2001; Gilchrist, Morrissey et al. 2003). The recombinant growth factors GDF9 and/or BMP15 are also capable of stimulating GC proliferation (McNatty, Juengel et al. 2005a\&b). However, care must be taken when extrapolating data from one species to another, as differences were noted with respect to the species of origin of the growth factors and/or species of GC. Moreover, species differences exist with respect to the roles of oocyte-derived GDF9 and BMP15 in ovarian follicular development and/or ovulation-rate (Juengel and McNatty 2005). The main objective of the experiments in this chapter was to determine whether, under in vitro conditions, rat and sheep oocytes were capable of stimulating GC proliferation in rat and sheep GC respectively. A second objective was to optimise the bioassay conditions for co-incubation of oocytes and GC in order to later test species differences in the roles of GDF9 and BMP15. Studies to address the second objective included evaluating the optimal number of oocytes and volume of media within each co-incubation well.

\subsection{Methodology}

The full details can be found in the general methodology section (Chapter 2.1-2.5). The DO were prepared as described in Chapter 2.1. Different numbers of DO were 
titrated with a constant number of GC in a series of co-incubation experiments. Each co-incubation had $0,8,32$ or 64 DO per well, and wherever possible, duplicates for each number of DO were included within an experiment. The DO-GC co-incubations were for $18 \mathrm{hrs}$ before the addition of methyl ${ }^{3} \mathrm{H}$-thymidine (see Chapter 2.5 ). Thereafter, the amount of ${ }^{3} \mathrm{H}$-thymidine incorporated into the GC over a $6 \mathrm{~h}$ period was used as an indirect measure of GC proliferation. A Trilux betacounter was used to measure ${ }^{3} \mathrm{H}$-thymidine incorporation.

Results from individual experiments were averaged and a one-way ANOVA was performed. Bonferroni's post-hoc test was performed for each pair of means if there were significant differences in the overall means. If the data were not normally distributed, as assessed by Levene's test, they were naturally log-transformed before analyses. In addition, the Kruskal-Wallis nonparametric test was often performed when the data were not normally distributed. Means that differed by $\mathrm{P}<0.05$ were considered to be significantly different from one another.

\subsection{Results}

The amounts of ${ }^{3} \mathrm{H}$-thymidine incorporated in rat $\mathrm{GC}$ after co-incubation with different numbers of rat DO are summarised in Figures $3 \mathrm{~A}$ and $\mathrm{B}$. The results indicate that rat DO stimulated ${ }^{3} \mathrm{H}$-thymidine incorporation by rat $\mathrm{GC}(\mathrm{N}=10$ individual experiments). Analyses of the log-transformed data showed that there were significant increases in ${ }^{3} \mathrm{H}$-thymidine incorporation in the presence of 32 or 64 DO compared with no DO, as well as between 64 and 8 DO. Experiment number 80228 could be considered to be an outlier because its fold changes were much greater than the others. However, it was performed under the same experimental conditions as the others and 
therefore there were no reasons for removing this data set from the analysis. Moreover, when the same analysis, using ANOVA was performed on natural log transformed data, without the results from Experiment 8028, the overall statistical effect remained unchanged $(\mathrm{P}<0.001)$. The inherent biological variability between the experiments contributes to the large confidence intervals.

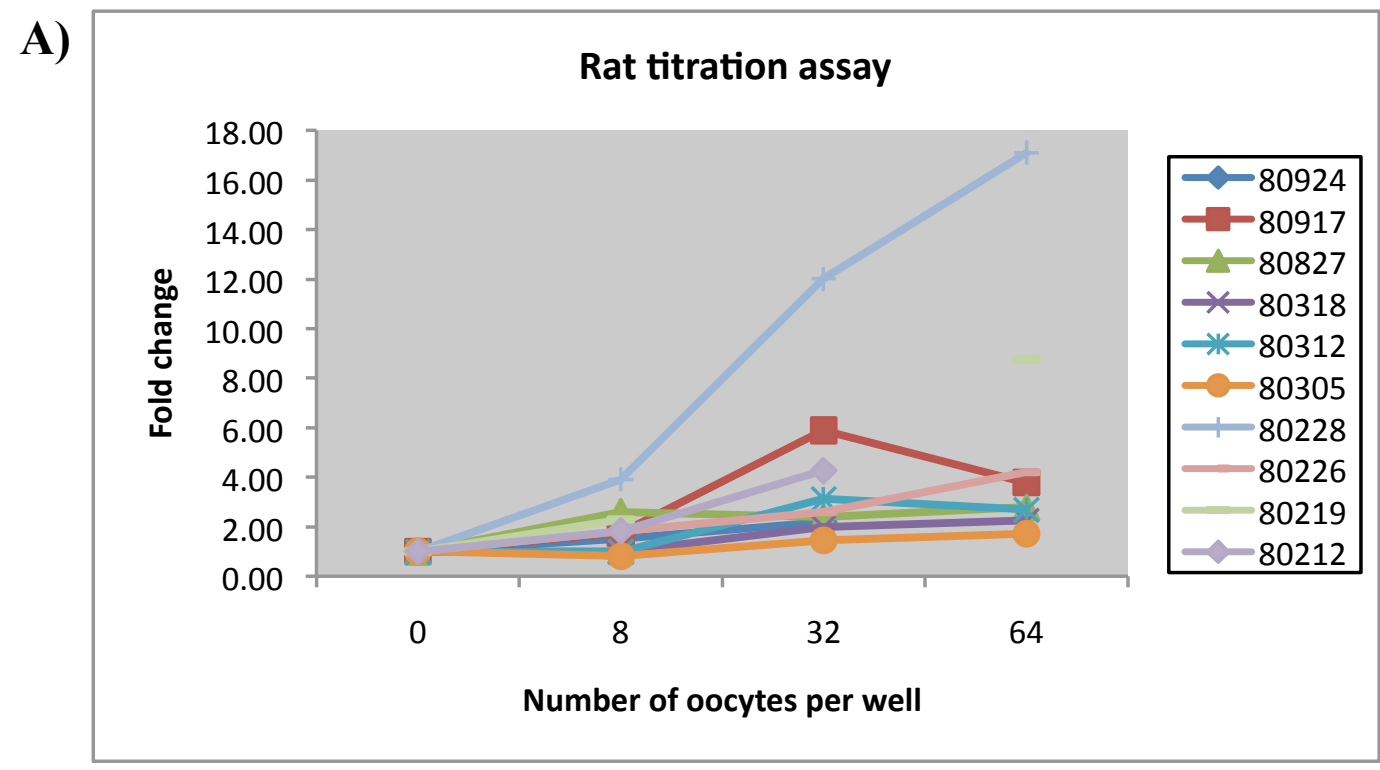

B)

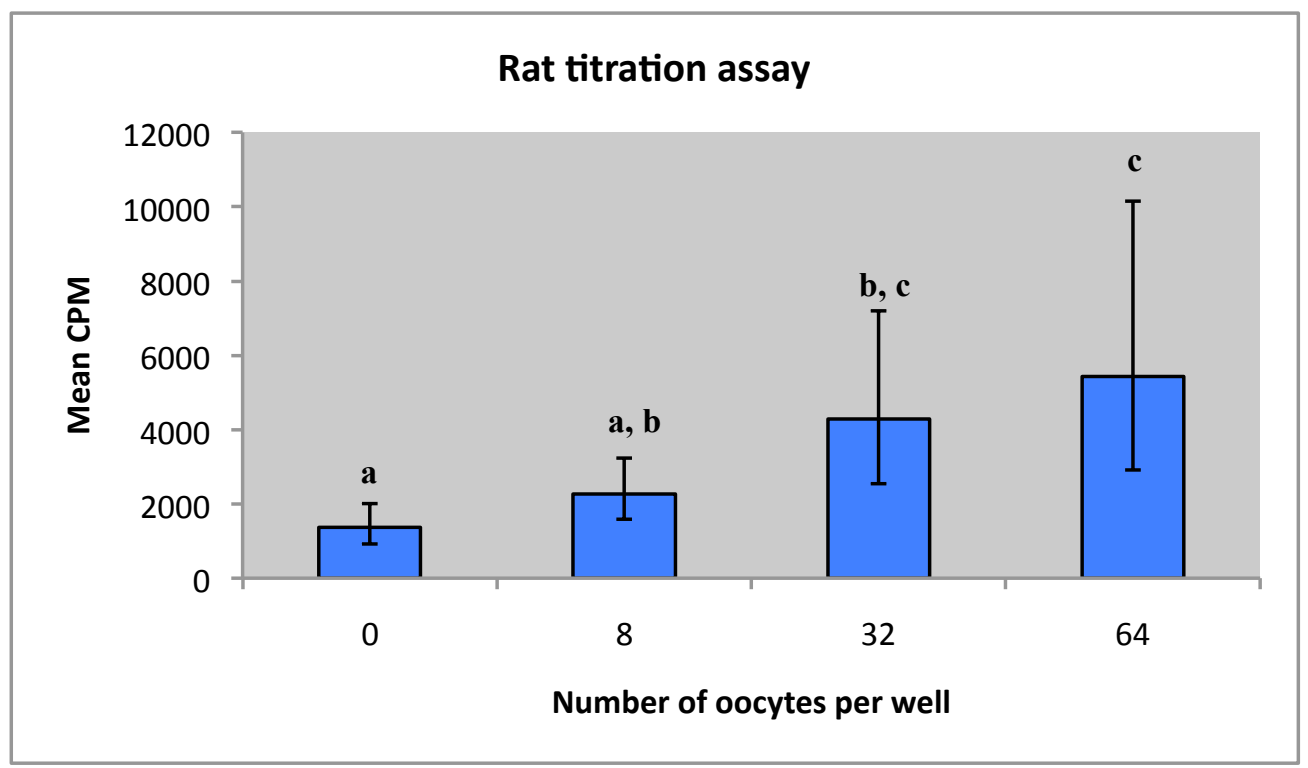

Figure 3A and B: Fold changes for individual experiments (A) and the overall mean effect of rat DO on ${ }^{3} \mathrm{H}$-thymidine incorporation in rat GC (B). The columns represent the geometric mean ${ }^{3} \mathrm{H}-$ thymidine incorporations and the vertical lines $\pm 95 \%$ confidence intervals. Columns not sharing an alphabetical letter are different from one another, $\mathbf{P}<0.05$. $C P M=$ counts per minute. 
As in rats, sheep DO stimulated sheep GC ${ }^{3} \mathrm{H}$-thymidine incorporation in a dosedependent manner (Figures $3 \mathrm{C}$ and $\mathrm{D}$ below, $\mathrm{N}=9$ individual experiments). The fold changes in each individual experiment are shown in Figure 3C. The general trend within each experiment was a dose dependent increase in GC proliferation as the number of DO per well increased (Figure 3D). The overall level of significance was $\mathrm{P}<0.001$. The inherent biological variability between the experiments contributes to the large confidence intervals.

Post-hoc analyses of the log-transformed data showed that there were significant differences between 0 and 8, 32 and 64 sheep DO with respect to ${ }^{3} \mathrm{H}$-thymidine incorporation in GC. In addition the mean value for 64 DO was significantly different to that for 8 DO (Figure 3D). 


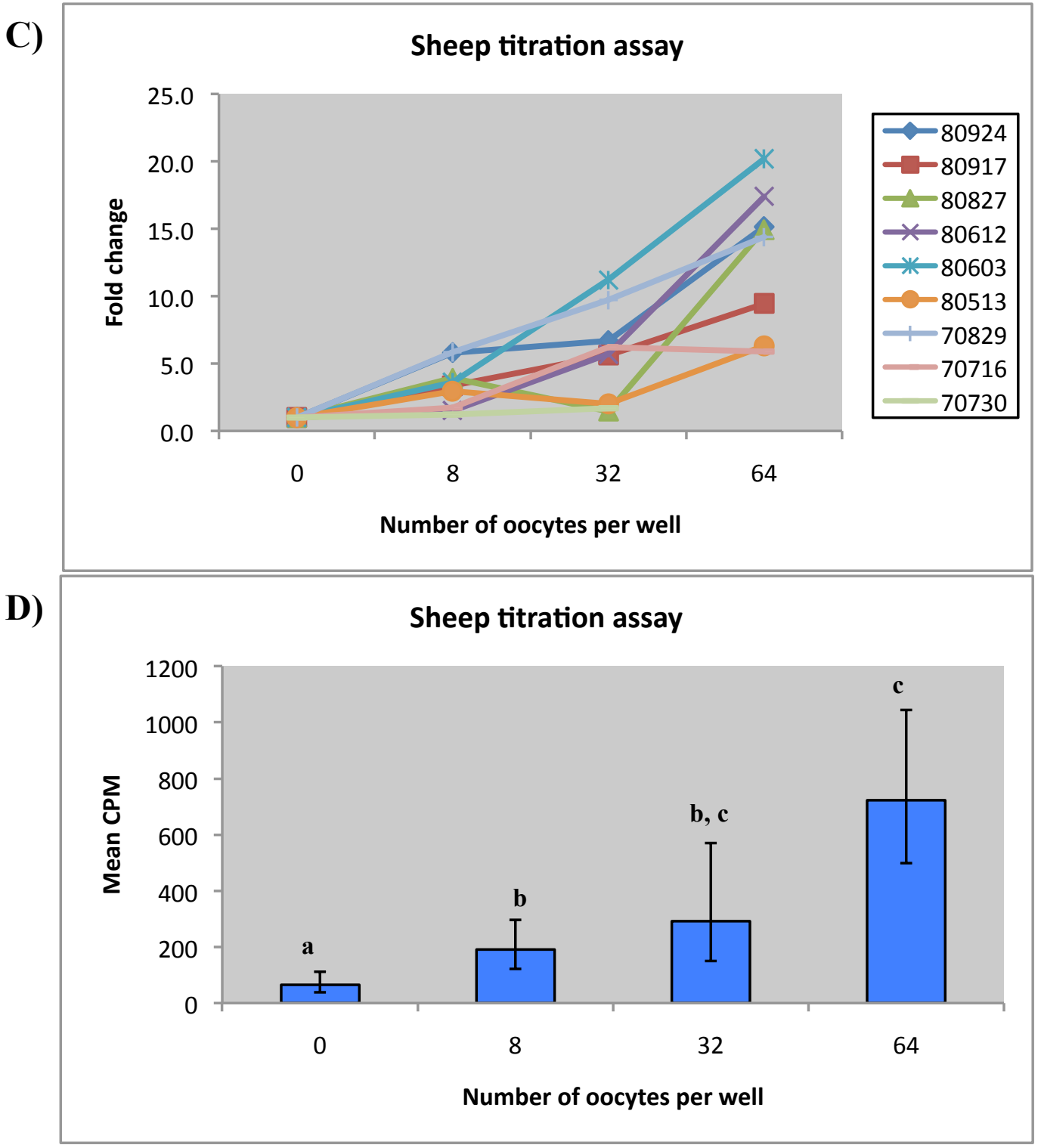

Figure 3C and D: Fold changes for the individual experiments $(C)$ and the overall mean effects of sheep DO on ${ }^{3} \mathrm{H}$-thymidine incorporation in sheep GC (D). The columns represent the geometric means and the vertical lines $\pm \mathbf{9 5 \%}$ confidence intervals. Columns not sharing a common alphabetical letter are significantly different from one another $(\mathrm{P}<0.05)$.

\subsection{Conclusion}

Rat DO showed a dose dependent stimulation of ${ }^{3} \mathrm{H}$-thymidine incorporation by rat GC and the increase was significant above the control with 32 and 64 DO per well. Sheep DO also showed a dose dependent stimulation in ${ }^{3} \mathrm{H}$-thymidine uptake by sheep GC and the increase was significant above the control when 8,32 or 64 sheep DO were used. 
Although Figures 3B and D show that the cpm for ${ }^{3} \mathrm{H}$-thymidine incorporation in rat GC were about 10 times higher than was the case for sheep GC,the mean fold change was greater in sheep with increasing numbers of oocytes (Figures $3 \mathrm{~A}$ and $\mathrm{C}$ ). This was due to the low background count for the sheep GC experiments compared to rat GC, which was a magnitude higher. The reason for this is not known. One possibility, however, might be that there is a higher basal rate of cell proliferation in rodents. For example, follicular growth in rats is about 6 times faster than in sheep (Peters and McNatty 1980).

The large confidence intervals seen in the Figures were due to the variability between experiments. The variability may, in part, have been due to the fact that at any moment in time no two follicles (including oocytes) are functionally identical (Peters et al. 1975), notwithstanding the effort made to collect oocytes of similar sizes and uniformity in morphology.

Although 8 DO from sheep were enough to significantly stimulate ${ }^{3} \mathrm{H}$-thymidine incorporation in sheep GC, 32 DO were decided upon in subsequent bioassays. With $32 \mathrm{DO}$, the mean level of ${ }^{3} \mathrm{H}$-thymidine incorporation was greater than when $8 \mathrm{DO}$ were used and not different from the stimulatory effects of 64 DO. Although the use of 64 DO may have been preferable given the inherent variability of the bioassays, it was impractical, on a routine basis, to undertake replicate experiments with this number. The same number of DO was decided for future bioassays in rats because 32 DO significantly stimulated ${ }^{3} \mathrm{H}$-thymidine uptake by rat $\mathrm{GC}$ and this was also a practical number to collect on a routine basis. With $32 \mathrm{DO}$, the mean fold increases in 
${ }^{3} \mathrm{H}$-thymidine incorporation in the two species was between 5-7 times that when no DO were present. 


\section{Chapter 4 CAN OOCYTES FROM A HIGH OVULATION- RATE SPECIES STIMULATE GC PROLIFERATION FROM A LOW OVULATION- RATE SPECIES AND VICE VERSA?}

\subsection{Introduction}

In both high and low ovulation-rate phenotypes, such as mice and sheep, inactivating GDF9 in vivo, either by mutation or immunization, prevented normal follicular growth and ovulation (Dong, Albertini et al. 1996; Carabatsos, Elvin et al. 1998; Yan, Wang et al. 2001; Juengel, Hudson et al. 2002; Hanrahan, Gregan et al. 2004; McNatty, Hudson et al. 2007; Nicol, Bishop et al. 2009). However, in mice, inactivating BMP15 had no significant effects on follicular development (Yan, Wang et al. 2001), whereas in sheep inactivating BMP15 inhibited normal follicular development and ovulation (Galloway, McNatty et al. 2000; McNatty, Juengel et al. 2001; Juengel, Hudson et al. 2002). In contrast, when BMP15 was only partially inactivated such as in the heterozygous mutant Inverdale, Hanna, Belclare or Cambridge ewes or by immunization, the ovulation-rate in sheep was increased by approximately $65-100 \%$ above that in the wild-types (Galloway, McNatty et al. 2000; McNatty, Juengel et al. 2001; Juengel, Hudson et al. 2002; Hanrahan, Gregan et al. 2004; Juengel, Hudson et al. 2004; McNatty, Hudson et al. 2007). Therefore, multiovulating species such as the rat or mouse, and low ovulation-rate species such as sheep are likely to have different requirements for BMP15 and GDF9 during follicular growth. Several studies have found that recombinant BMP15 and/or GDF9 of one species has the ability to stimulate GC proliferation of a different species in vitro (Otsuka, Yao et al. 2000; Kaivo-Oja, Bondestam et al. 2003; Lee, Yoon et al. 2004; 
Liao, Moore et al. 2004; McNatty, Juengel et al. 2005a\&b; McNatty, Lawrence et al. 2006; Sugiura, Su et al. 2007; Mottershead, Pulkki et al. 2008). Thus, if there are similarities across species in their responses to GDF9 and BMP15, a question arises as to whether oocytes from one species can stimulate GC proliferation from a species with a different ovulation-rate phenotype since, within a species and irrespective of ovulation-rate phenotype, oocytes can stimulate ${ }^{3} \mathrm{H}$-thymidine uptake by $\mathrm{GC}$ (see Chapter 3). Therefore, the aim of the experiments in this chapter was to establish whether sheep DO could stimulate ${ }^{3} \mathrm{H}$-thymidine incorporation in rat GC and vice versa.

\subsection{Methodology}

Briefly, to show whether DO were able to stimulate proliferation, as assessed by ${ }^{3} \mathrm{H}-$ thymidine incorporation in GC, DO of one species (sheep or rat) were co-incubated with a constant number of GC from the other species (rat or sheep). The numbers of oocytes added to each well were $0,8,32$ or 64 and the number of GC was kept constant at 20,000. The co-incubations were undertaken for $24 \mathrm{~h}$. For the last six hours of co-incubation, ${ }^{3} \mathrm{H}$-thymidine was added to each of the wells. A Trilux betacounter was used to measure ${ }^{3} \mathrm{H}$-thymidine incorporation. See the method section, 2.1, 2.4 and 2.5, for the full methodology.

Results from individual experiments were averaged and a one-way ANOVA was performed. If differences were observed from ANOVA, the Bonferroni's post-hoc test was used to test for differences between individual group means. If the data were not normally distributed, as assessed by Levene's test, they were naturally logtransformed before analyses. For non-normally distributed data, the Kruskal-Wallis 
non-parametric test was also undertaken. Means that differed by $\mathrm{P}<0.05$ were considered to be significantly different from one another.

\subsection{Results}

For rat DO co-incubated with sheep GC, the results in Figure 4A indicate that there are two different populations of responses ( $\mathrm{N}=$ seven individual experiments). Of these, three experimental data sets showed high levels of fold changes, whereas the other four experiments showed no fold changes. Therefore, the responses were separated accordingly and analysed separately. Of the experiments that did show high levels of fold change, 64 rat DO per well significantly stimulated ${ }^{3} \mathrm{H}$-thymidine incorporation in sheep GC, $(\mathrm{P}=0.05$; Figure $4 \mathrm{C})$. Kruskal-Wallis analyses of the remaining four experiments showed no significant stimulation, $\mathrm{P}=0.9$ (Figure 4D). Therefore, $43 \%$ of the time there was significant stimulation by rat DO of sheep GC but only when 64 rat DO were used. When all the results of all experiments were combined and analysed by Kruskal-Wallis, no significant ${ }^{3} \mathrm{H}$-thymidine incorporation by GC was noted (Figure 4B). 
A)

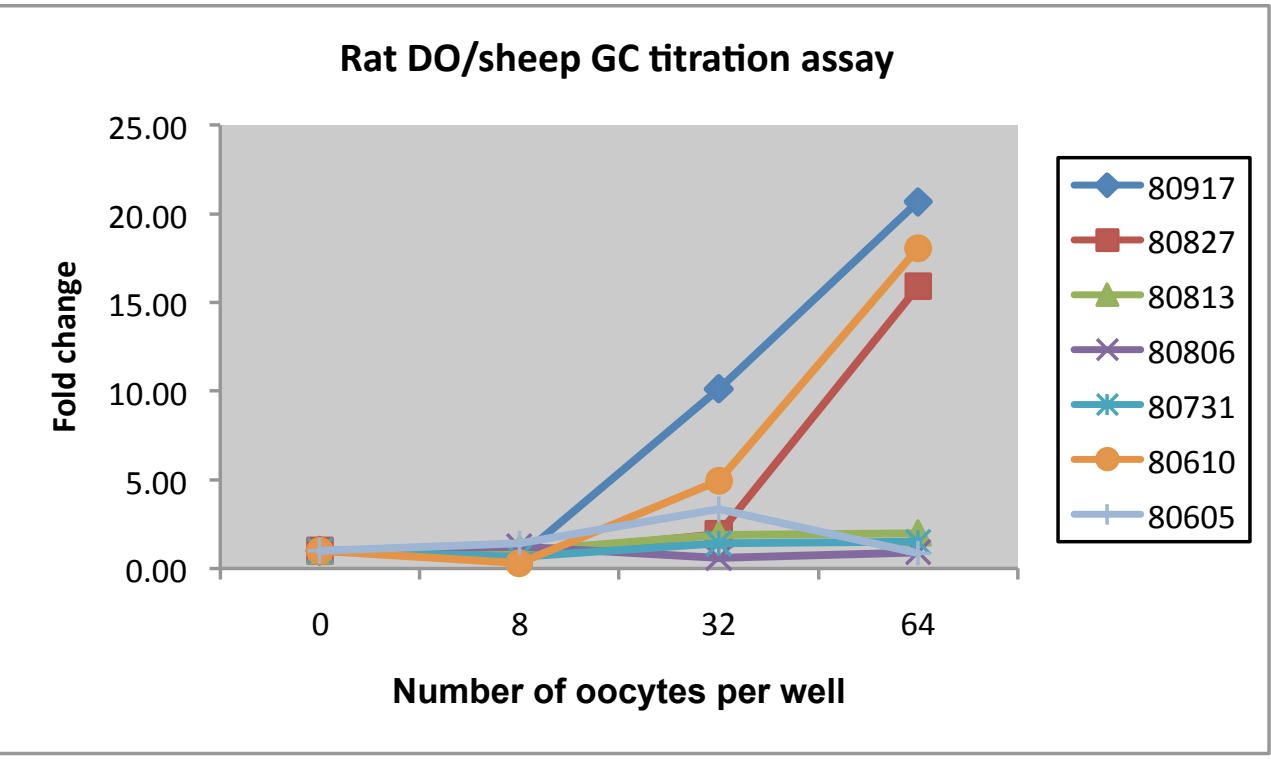

B)

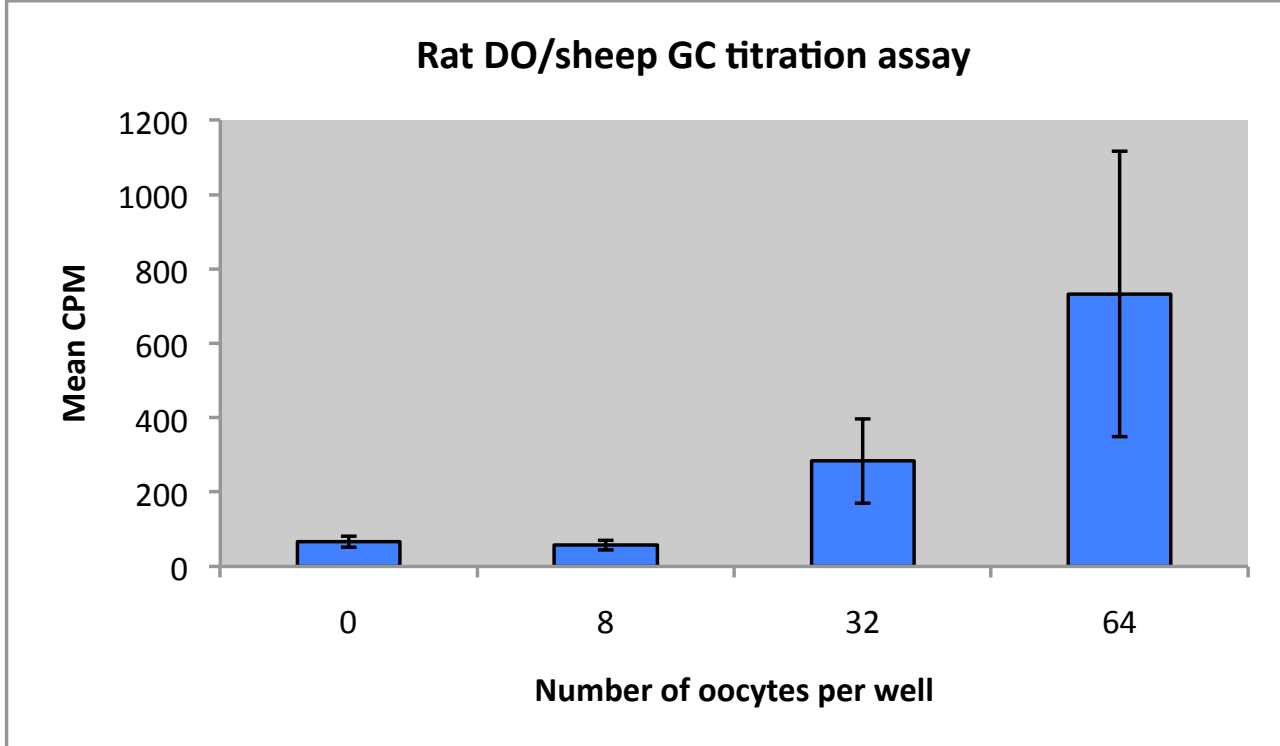

Figures 4A and B: Fold changes of individual experiments when rat DO were co-incubated with sheep GC (A) and the overall mean effects of rat DO on ${ }^{3} \mathrm{H}$-thymidine incorporation by sheep GC $(\mathrm{N}=7)(B)$. Columns represent means and the vertical lines \pm SEM. CPM=counts per minute. In Figure 4B no statistical difference was noted as assessed by the Kruskal-Wallis test. 
C)

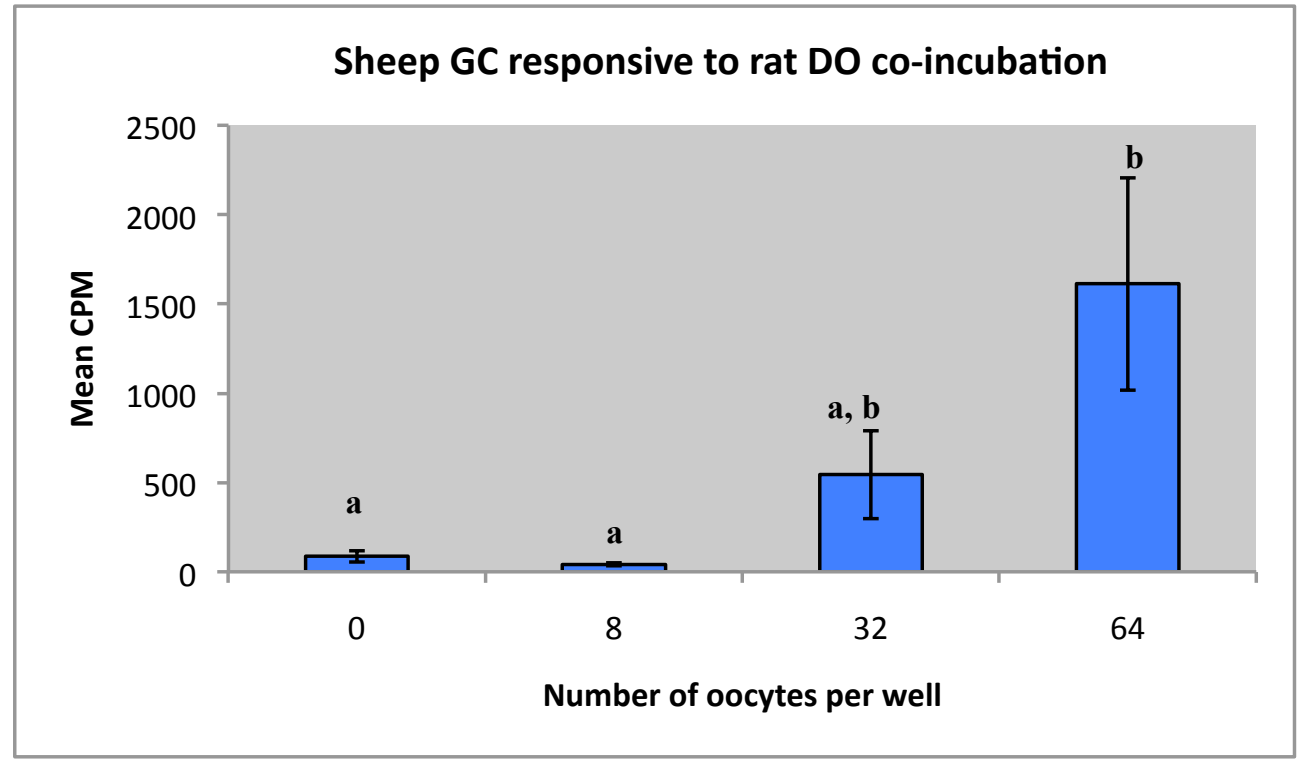

D)

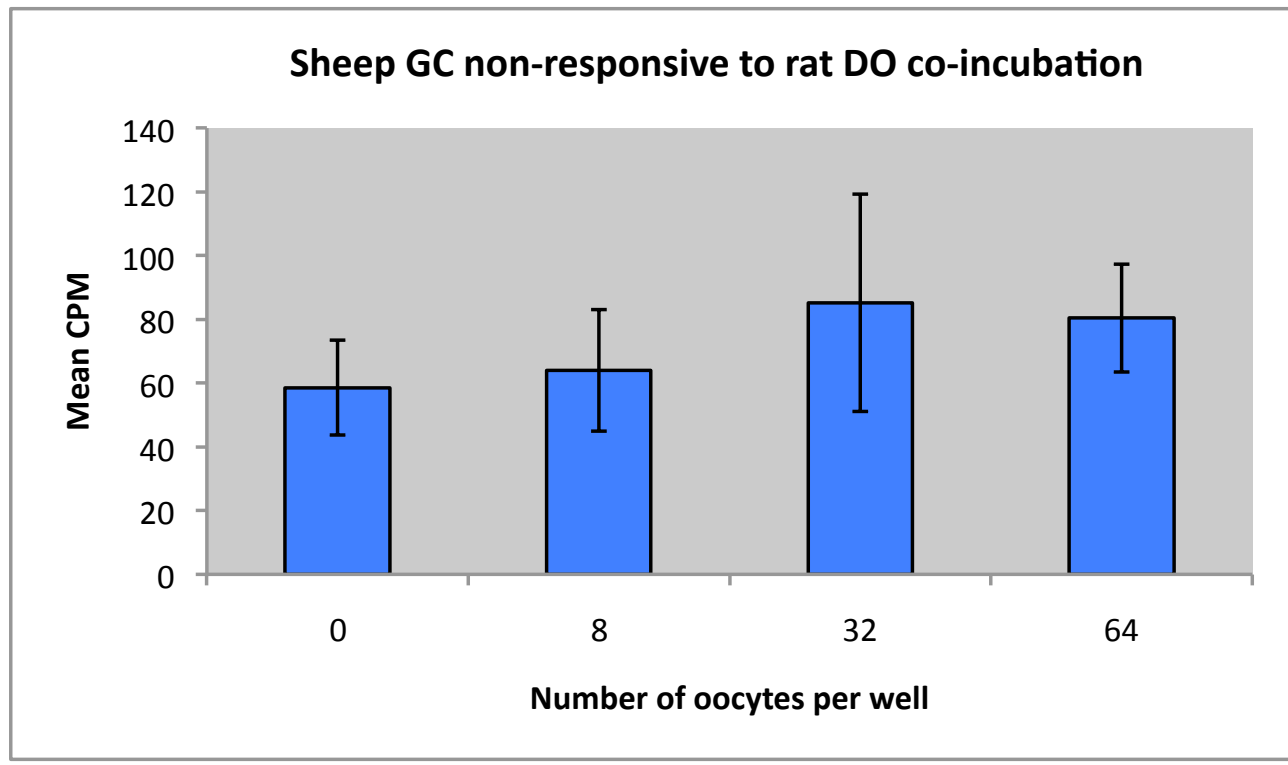

Figures 4C and D: Pooled means of rat DO co-incubated with sheep GC with a $>10$-fold increase in ${ }^{3} \mathrm{H}$-thymidine incorporation in GC noted when $64 \mathrm{DO}$ were used $(\mathrm{N}=3$ individual experiments; see Figure 4A) (C) and when there was <2-fold increase in ${ }^{3} \mathrm{H}$-thymidine incorporation in GC noted when 64 DO were used ( $N=4$ individual experiments; see Figure 4B) (D). Columns represent means and vertical lines \pm SEM. In Figure $4 \mathrm{C}$, columns not sharing a common alphabetical letter are significantly different from one another $P<0.05$. In Figure 4D no significant differences were noted.

Figure 4E shows the fold changes for the nine independent experiments of sheep DO co-incubated with rat GC. Of the nine, experiment number 80806 was removed from the overall analysis because it was a non-responding outlier. This was tested by Grubb's test for outliers. However when the data from this experiment was included, it did not impact on the overall level of significance. Therefore $87.5 \%$ of the 
experiments showed an increasing fold change with increasing numbers of DO. There was significant stimulation of ${ }^{3} \mathrm{H}$-thymidine incorporation by rat $\mathrm{GC}$ when 8,32 , or 64 DO were used $(\mathrm{P}<0.01$; Figure $4 \mathrm{~F})$. Furthermore, co-incubation with 64 DO was significantly greater than when $8 \mathrm{DO}$ were used (Figure 4F).
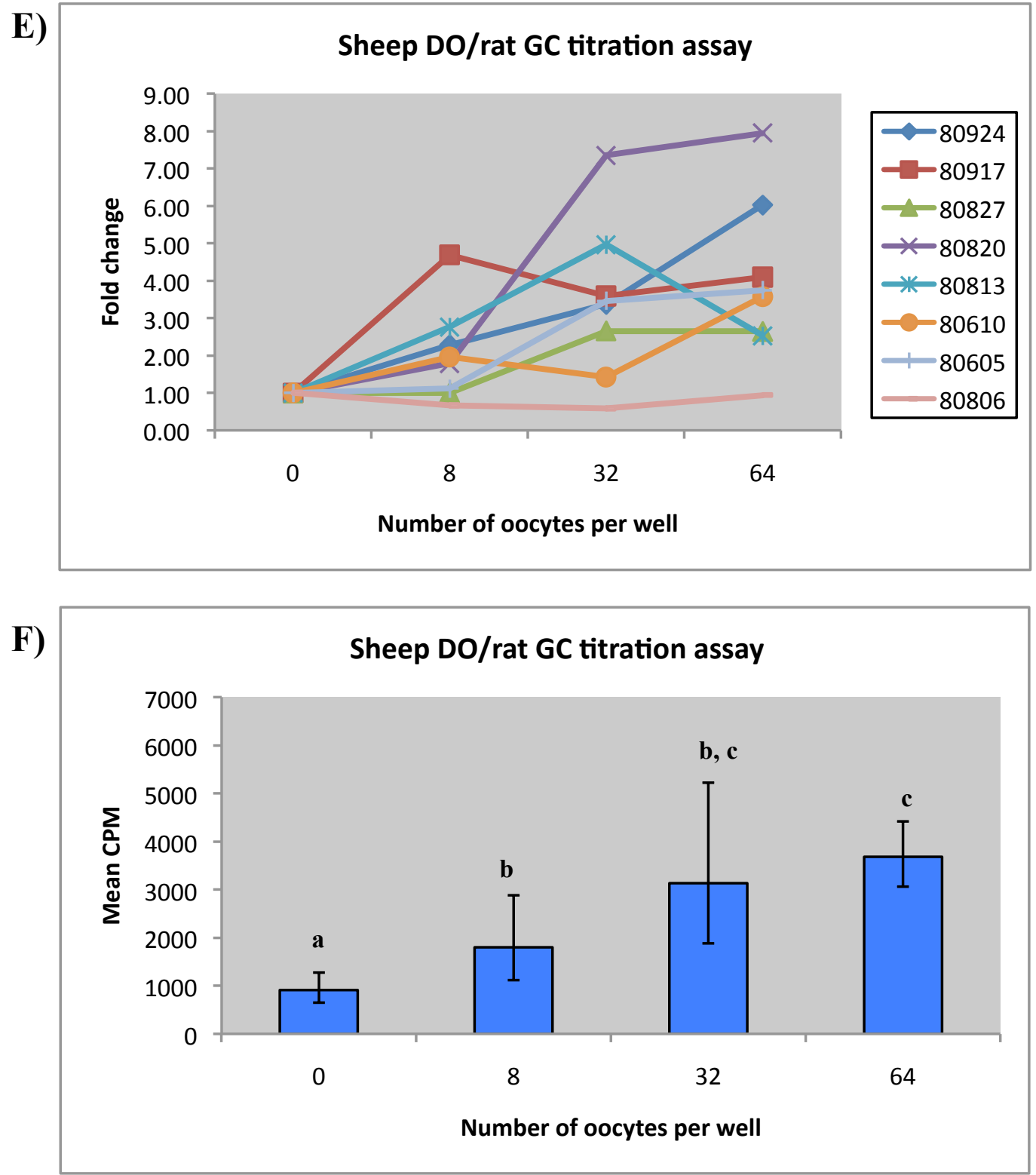

Figures 4E and F: Fold changes of individual experiments when sheep DO were co-incubated with rat GC (E) and the overall mean effects of sheep DO on ${ }^{3} \mathrm{H}$-thymidine incorporation by rat GC (F). Columns represent means and the vertical lines \pm SEM. Columns not sharing a common alphabetical letter are significantly different from one another, $\mathrm{P}<0.05$. 


\subsection{Conclusion}

In general, sheep DO significantly stimulated ${ }^{3} \mathrm{H}$-thymidine incorporation by rat GC. The level of ${ }^{3} \mathrm{H}$-thymidine incorporation was significant when 8,32 or 64 DO were used indicating that a limited number of sheep DO were sufficient to stimulate proliferation of rat GC.

In contrast, rat DO were not able to reproducibly stimulate ${ }^{3} \mathrm{H}$-thymidine incorporation by sheep GC. On some occasions (i.e. 3/7) 64 but not 32 or fewer DO significantly stimulated ${ }^{3} \mathrm{H}$-thymidine incorporation. All 7 experiments were carried out using the same protocols, as detailed in Sections, 2.1, 2.4 and 2.5. As discussed in Chapter 3, the oocytes are recovered from follicles that at any given time are at a different stage of development (Peters \& McNatty, 1980). Thus, it would be reasonable to expect differences in the amount of GDF9 and BMP15 (and other growth factors not within the scope of this study) produced by individual oocytes within each pool. Moreover, previous studies have shown that very small changes in the concentrations of recombinant BMP15 relative to GDF9 protein, or vice versa, can cause large changes (e.g. 7-fold) in ${ }^{3} \mathrm{H}$-thymidine uptake by GC (McNatty, Juengel et al, 2005a; McIntosh, Lun et al. 2008; Reader, Heath et al. 2011). This, in turn, may explain why an increase in ${ }^{3} \mathrm{H}$-thymidine incorporation was noted in some but not all replicate experiments in which sheep GC was co-incubated with 64 rat DO.

To investigate whether rat DO lack sufficient BMP15 to stimulate sheep GC proliferation, co-incubations of rat DO-sheep GC with recombinant sheep BMP15 produced by transfected $293 \mathrm{H}$ cells were tested. However, the $293 \mathrm{H}$ media had an inhibitory effect on ${ }^{3} \mathrm{H}$-thymidine incorporation by sheep GC. Thus, it was not 
possible to test if exogenous BMP15 would have helped rat DO to stimulate ${ }^{3} \mathrm{H}$ thymidine incorporation by sheep GC. At the time of undertaking these experiments, no purified BMP15 (or GDF9) reagents were available.

In light of the results from Chapters 3 and herein, the next step was to test if GDF9 and/or BMP15 were involved in the stimulatory effects of oocytes on GC proliferation. In the subsequent experiments, $32 \mathrm{DO}$ were used for either species as this number was sufficient to cause significant stimulation of ${ }^{3} \mathrm{H}$-thymidine incorporation. Importantly, 32 DO per test was also a reasonable number to collect within a satisfactory time frame. Co-incubations of rat DO with sheep GC were not undertaken in subsequent experiments given the inability to consistently see an effect with 32-64 DO per test. 


\section{Chapter 5 EFFECT OF IMMUNO-NEUTRALISATION OF OOCYTE-DERIVED GDF9 OR BMP15 ON ${ }^{3} \mathrm{H}-$ THYMIDINE INCORPORATION BY GC}

\subsection{Introduction}

In Chapter 3, it was established that oocytes could, dose-dependently, stimulate ${ }^{3} \mathrm{H}$ thymidine incorporation in GC when the same species of GC were used. Moreover, it was established that an appropriate number of DO per well to demonstrate this was 32. In Chapter 4, it was established that sheep oocytes could also dose-dependently stimulate ${ }^{3} \mathrm{H}$-thymidine incorporation in rat GC and that an appropriate number of DO to reproducibly demonstrate this was also 32. It was also established that rat oocytes were unable to significantly stimulate ${ }^{3} \mathrm{H}$-thymidine incorporation in sheep GC unless a very large number of rat DO were used. However, even with 64 DO per well, significant ${ }^{3} \mathrm{H}$-thymidine incorporation was only achieved $<50 \%$ of the time. Therefore, with the exception of co-incubations of rat DO with sheep GC, all following bioassays were undertaken with $32 \mathrm{DO}$ per well. Co-incubations of rat DO with sheep GC was not continued because it was not practical to set up experiments with the extremely large number of oocytes required and without consistency with respect to a significant increase in ${ }^{3} \mathrm{H}$-thymidine incorporation

Having established that oocytes do have the ability to stimulate ${ }^{3} \mathrm{H}$-thymidine incorporation in GC, the next step was to examine if the effects were due to GDF9 and/or BMP15. To test whether these growth factors were involved, GDF9 or BMP15-specific antibodies were added to the co-incubations. For the control experiments, a monoclonal antibody to a nematode worm (MabPAB1) was used. 


\subsection{Methodology}

Incubations were set up as described in the methods section. DO were transferred in $30 \mu 1$ of M199 media, 20 $\mu 1$ of GC (20,000 cells) were added and the antibodies were added in a $5 \mu 1$ aliquot: the total volume for each incubation experiment was $55 \mu 1 /$ well. Each experiment included replicate wells with specific monoclonal antibodies to GDF9 or BMP15 or a nematode (Hydatids) antigen (i.e. control) and a no antibody control was also included. At least three independent experiments were undertaken for each of the treatments.

The monoclonal antibody 47B (Mab47B) was generated by Oxford Brookes University under contract to AgResearch, against the sheep GDF9 amino acid cterminus sequence, SEYFKQFLFPQNEC. This also had 100\% sequence homology to the corresponding region in mice. It has previously been reported that this antibody recognizes the mouse GDF9 mature protein in Western blotting and in immunoneutralisation experiments (McIntosh, Lun et al. 2008). The c-terminus of GDF9 is highly conserved and the sheep sequence has $93 \%$ homology with the corresponding rat GDF9 sequence, SEYFRQFLFPQNEC, where only one amino acid differed (in bold) between the two sequences. In preliminary experiments a series of concentrations were tested. Following these studies a concentration of $14.6 \mu \mathrm{g} / \mathrm{ml}$ of Mab47B was selected for rat DO with rat GC and 7.6 $\mu \mathrm{g} / \mathrm{ml}$ of Mab47B was selected for co-incubations of sheep DO with sheep or rat GC.

The BMP15 monoclonal antibody 61A (Mab61A) used in these experiments was also made by Oxford Brookes University under contract to AgResearch. The antibody was 
generated after the immunization of mice against an E. coli-derived sheep BMP15 mature protein. The monoclonal antibody activity seems to be directed against a sequence on the N-terminus of the mature protein, QAGSIASEVPGPSR (Dr Juengel, unpublished data). This N-terminal peptide was known to inhibit ovarian function in sheep in vivo (McNatty, Hudson et al. 2007) when animals were vaccinated against this material conjugated to KLH. This sequence has a $64 \%$ homology with the corresponding rat BMP15 sequence, QTCSIASDVPCPSQ (amino acid differences are in bold). However, there are four homologous amino acids in a row (italicised) and previous studies indicate that a $27 \%$ homology can be sufficient for antibody recognition of ligand (Gilchrist, Ritter et al. 2004; McNatty, Hudson et al. 2007). The ability of Mab61A to recognise rat BMP15 protein was confirmed by Western blotting (see Chapter 6). After testing a series of concentrations $(0-18 \mu \mathrm{g} / \mathrm{ml})$, a $3.2 \mu \mathrm{g} / \mathrm{ml}$ concentration of Mab61A was selected. At this concentration, it was expected that the degree of inhibition of BMP15 (if present) would be $\sim 50 \%$ or more (McNatty, Juengel et al. 2005a and b). Thus, based on the species homology and the preliminary titrations, it was reasonable to expect that the chosen antibodies would be appropriate for the proposed experiments.

Results from the replicated experiments were collected and averaged. The differences in ${ }^{3} \mathrm{H}$-thymidine incorporation between $32 \mathrm{DO}$ and 0 DO for each treatment was taken and a percentage of each treatment against the no antibody control was calculated. An independent student t-test was used to test for significance between the specific antibody and the control antibody treatments. When the data were not normally distributed, the non-parametric Mann-Whitney test was performed. The different tests 
used were because paired means were compared as opposed to multiple means in Chapters 3-4. Means that differed by $\mathrm{P}<0.05$ were deemed significant.

\subsection{Results}

An initial experiment was carried out to test if concentrations from $3.2 \mu \mathrm{g} / \mathrm{ml}$ to $18 \mu \mathrm{g} / \mathrm{ml}$ of the control antibody affected ${ }^{3} \mathrm{H}$-thymidine incorporation by GC. There was no significant change between the concentrations tested and the no antibody control (data not shown). Table 5A shows the raw data of the control antibody (PAB1) versus the no antibody control (none). There was no significant effect of PAB1 on ${ }^{3} \mathrm{H}$-thymidine uptake when compared to the no antibody treatment. Consequently, the effect of each of the treatments, i.e. GDF9, BMP15, and PAB1 antibody, were calculated relative to the effect of the no antibody treatment.

Table 5A: Effect of control antibody (PAB1) and no antibody control (none) on ${ }^{3} \mathrm{H}-$ thymidine incorporation by GC following a $24 \mathrm{~h}$ DO-GC co-incubation. Cross-species refers to sheep DO with rat GC whereas the rat and sheep results are for the homologous co-incubations. Values are shown as Mean $\pm \mathrm{SEM}$ counts per minute.

\begin{tabular}{|c|c|r|r|r|r|r|r|}
\cline { 3 - 8 } \multicolumn{2}{c|}{} & \multicolumn{6}{c|}{ Cell combinations } \\
\cline { 3 - 8 } \multicolumn{2}{c|}{} & \multicolumn{2}{c|}{ Rat } & \multicolumn{2}{c|}{ Sheep } & \multicolumn{1}{c|}{ Cross Species } \\
\hline Treatment & No. of DO & Mean & \multicolumn{1}{c|}{ SEM } & Mean & \multicolumn{1}{c}{ SEM } & Mean & \multicolumn{1}{c}{ SEM } \\
\hline PAB1 & 0 & 342 & 115 & 88 & 15 & 423 & 211 \\
& 32 & 2264 & 923 & 491 & 51 & 3562 & 1392 \\
None & 0 & 287 & 87 & 69 & 9 & 321 & 121 \\
& 32 & 2033 & 630 & 497 & 173 & 3921 & 1704 \\
\hline
\end{tabular}

Figure 5A shows the effect of neutralising oocyte-derived GDF9 protein on ${ }^{3} \mathrm{H}$ thymidine incorporation by GC. In all cases, there was a significant reduction in ${ }^{3} \mathrm{H}$ thymidine incorporation. In rat DO with rat GC, there was $\sim 55 \%$ reduction in ${ }^{3} \mathrm{H}$ thymidine incorporation as compared to that for the control antibody $(\mathrm{P}<0.05)$. In sheep DO with sheep GC, there was a 50\% reduction $(\mathrm{P}<0.05)$ and with rat GC, there was $\sim 75 \%$ reduction $(\mathrm{P}<0.02)$ in ${ }^{3} \mathrm{H}$-thymidine incorporation. 


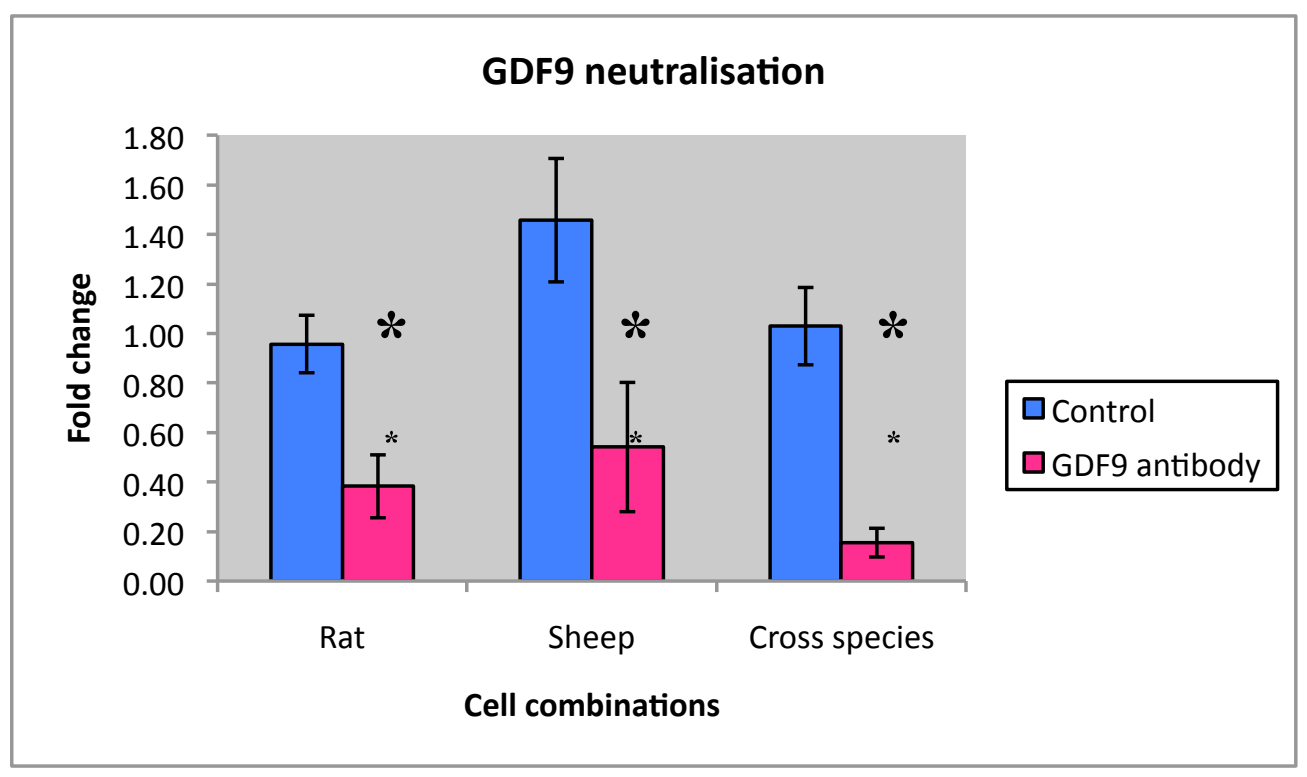

Figure 5A: Effect of control or GDF9 monoclonal antibody on ${ }^{3} \mathrm{H}$-thymidine incorporation by GC following a 24h DO-GC co-incubation. Cross-species refers to sheep DO with rat GC whereas the rat and sheep results are for the homologous co-incubations. Columns represent mean fold change compared to no antibody control and the vertical lines represent \pm SEM. Asterisks indicate statistical significance as compared to the control antibody, $P<0.05$.

Figure 5B summarises the neutralising effects of oocyte-derived BMP15 protein. Rat oocytes co-incubated with rat GC showed no reduction in ${ }^{3} \mathrm{H}$-thymidine incorporation by GC $(\mathrm{P}=0.988)$. However, sheep oocytes with sheep GC or rat GC showed $\sim 70 \%$ $(\mathrm{P}<0.05)$ or $\sim 50 \%(\mathrm{P}<0.01)$ reduction in ${ }^{3} \mathrm{H}$-thymidine incorporation respectively. 


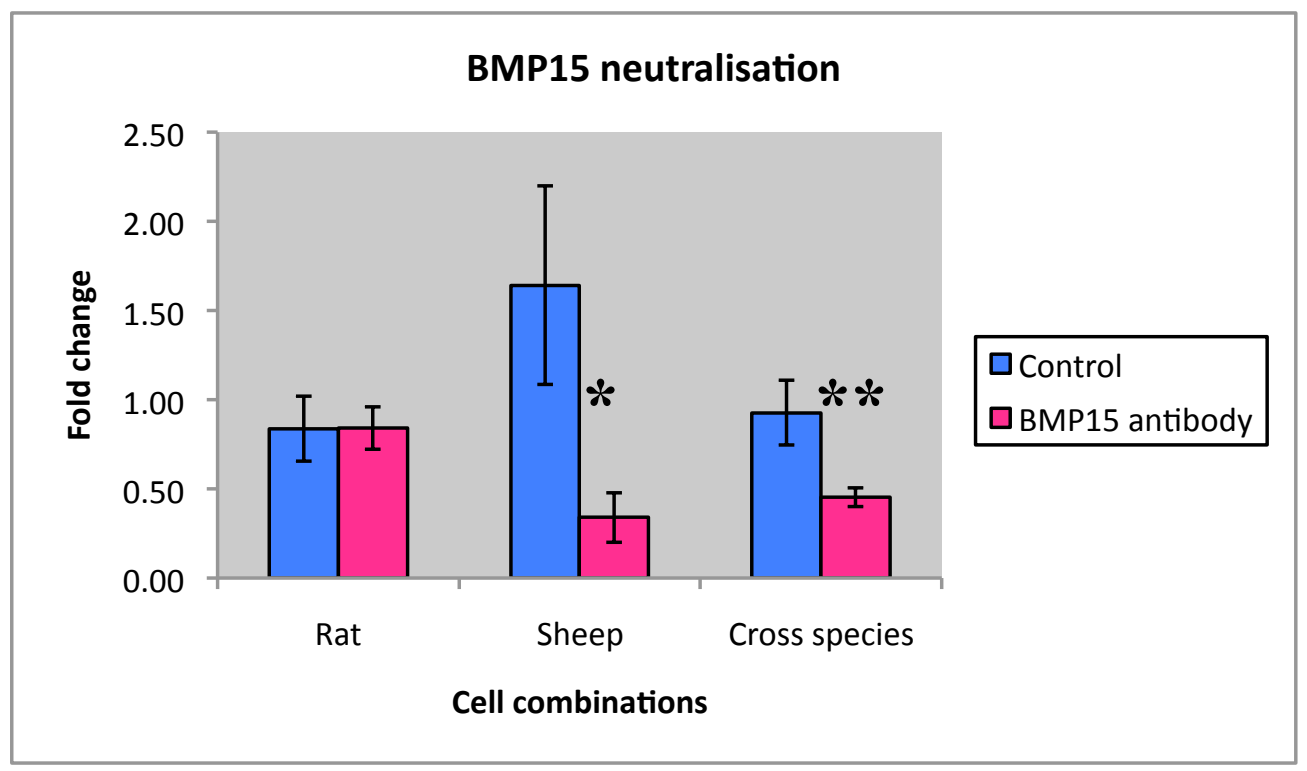

Figure 5B: Effect of control or BMP15-specific monoclonal antibody on ${ }^{3} \mathrm{H}-$ thymidine incorporation by GC following a $24 \mathrm{~h}$ DO-GC co-incubation. Columns represent mean fold change compared to no antibody control and the vertical lines represent \pm SEM. Asterisks indicates statistical significance as compared to the control antibody, $* \mathrm{P}<0.05$ for sheep and $* * \mathrm{P}<0.01$ for cross-species (i.e. sheep DO and rat $\mathrm{GC}$ ).

\subsection{Conclusion}

The addition of the GDF9 monoclonal antibody (Mab47B) resulted in reduced ${ }^{3} \mathrm{H}$ thymidine incorporation by GC in all the species combinations tested, indicating that oocyte-derived GDF9 protein is a key factor for proliferation of GC for both rats and sheep. The fact that a higher concentration of GDF9 antibody was used to neutralise oocyte-derived GDF9 protein in rat incubations may indicate more GDF9 protein was present in rat bioassays, or more likely the antibody was not optimal against rat GDF9 protein as it was made against a sheep GDF9 c-terminal sequence. However, as there were no other bioactive GDF9 antibodies available, this was the best option available at the time the experiments were undertaken.

Neutralising oocyte-derived BMP15 protein with Mab61A also resulted in reduced

${ }^{3} \mathrm{H}$-thymidine incorporation by GC in co-incubations of sheep DO with sheep GC or 
rat GC. However, it did not result in any significant reduction in ${ }^{3} \mathrm{H}$-thymidine incorporation by rat GC co-incubated with rat DO. It is possible that the rat oocytes produced BMP15 proteins but could not be neutralised by the antibody, although the monoclonal antibody used to immuno-neutralise BMP15 bioactivity in the rat coincubation experiments recognised rat BMP15 protein by Western blotting methods (see Chapter 6). An alternative explanation is that rat oocytes produce very little BMP15 protein and that GC proliferation is regulated by GDF9.

The BMP15 immuno-neutralisation experiments showed clearly that BMP15 secreted by sheep oocytes is a key growth factor in stimulating ${ }^{3} \mathrm{H}$-thymidine incorporation by sheep GC. Taken together with the failure of many pools of rat DO to stimulate ${ }^{3} \mathrm{H}-$ thymidine incorporation in sheep GC, it appears that sheep GC require both GDF9 and BMP15 to stimulate ${ }^{3} \mathrm{H}$-thymidine incorporation in contrast to rat GC which appear to require only GDF9.

Unfortunately, due to the limited amount of antibodies available, further experiments to test whether these growth factors alone were capable of completely inhibiting ${ }^{3} \mathrm{H}$ thymidine incorporation, were not conducted. However, it is reasonable to expect this could have been achieved if higher concentrations of antibody were used, since sheep with homozygous mutations in GDF9 or BMP15, sheep immunised against GDF9 or BMP15 (Juengel, Husdon et al. 2002) or GDF9 but not BMP15 knockout mice (Yan, Wang et al. 2001) experience no ovarian follicular growth beyond the primary stage of growth. 


\section{$\begin{array}{llllll}\text { Chapter } 6 \text { MOLECULAR FORMS OF GDF9 AND } & \text { BMP15 }\end{array}$ PROTEINS IN OOCYTE LYSATES AND SPENT MEDIA}

\subsection{Introduction}

In Chapter 5, the results indicated the importance of oocyte-derived GDF9 and/or BMP15 in stimulating ${ }^{3} \mathrm{H}$-thymidine incorporation by GC. These data suggest that BMP15 and/or GDF9 proteins are synthesized and secreted by the oocytes. Thus the aim of these studies was to determine whether GDF9 and BMP15 proteins could be identified in oocytes and the incubation media and to identify the molecular forms. A key objective was to establish whether the results of these studies would be consistent with the findings reported in Chapters 3, 4 and 5. The oocyte-derived growth factors GDF9 and BMP15 are members of the TGF- $\beta$ superfamily which are usually characterised by the production of pro-mature proteins that are cleaved to generate separate pro- and mature regions (McPherron and Lee 1993; McGrath, Esquela et al. 1995; Bodensteiner, Clay et al. 1999; Jaatinen, Laitinen et al. 1999; Galloway, McNatty et al. 2000; Juengel, Bodensteiner et al. 2004; Knight and Glister 2006). In vivo, unprocessed pro-mature and/or mature forms of GDF9 and BMP15 proteins have been found in follicular fluid of women, monkeys and sheep (Duffy 2003; McNatty, Lawrence et al. 2006; Wu, Tang et al. 2007; Shi, Cheung et al. 2009). However, a search of the current literature found only one paper by Gilchrist, Ritter et al. (2004) which showed GDF9 pro-mature and mature monomer forms in cultured mouse oocyte lysates and the spent medium. In the present study, Western blotting procedures were used to identify possible pro-mature and/or mature regions of GDF9 and BMP15 in rat and sheep oocyte lysates and spent media after $24 \mathrm{~h}$ of incubation. 
For the sheep GDF9 Western blot experiments, the GDF9 monoclonal antibody 37A (Mab37A) was used. This antibody has been shown to be specific for sheep GDF9 in previous Western blots performed within our laboratory (McNatty, Juengel et al. 2005a). Mab37A was generated by AgResearch staff in the Groome laboratory at Oxford Brookes University, UK against an E. coli-generated mature sheep GDF9 protein that was prepared in the Juengel, McNatty laboratory at AgResearch, Wallaceville. However, the ability of this antibody to inhibit GDF9 bioactivity in cell culture had not previously been established. For the rat, the monoclonal antibody 47B (Mab47B) was used. This antibody was generated against the common GDF9 mature protein sequence in sheep and the mouse, SEYFKQFLFPQNEC. Mab47B had previously been shown to recognise both mouse and sheep recombinant GDF9 and to immuno-neutralise the bioactivity of these recombinant forms of GDF9 in, in vitro bioassays (McIntosh, Lun et al. 2008). In the rat, the corresponding amino acid sequence is SEYFRQFLFPQNEC indicating a 93\% homology with the mouse and sheep sequences (the amino acid that differs to the sheep and mouse sequence is in bold). Thus, there was a strong likelihood, based on the degree of homology, that Mab47B would be useful for detecting rat GDF9. The current Western blots of sheep GDF9 were carried out prior to the immuno-neutralisation assays in Chapter 5.

With respect to BMP15, a key question was whether Mab61A, which was used to immnuo-neutralise rat BMP15 in Chapter 5, was capable of recognising this growth factor in the rat. Mab61A was generated against the sheep amino acid sequence QAGSIASEVPGPSR. The corresponding sequence in the rat is QTCSIASDVPCPSQ. Thus to further characterize this, Western blots of Mab61A was performed and compared with another monoclonal antibody $3 \mathrm{~A} / \mathrm{A}$. Worldwide there are currently no 
reliable commercially available antibodies for BMP15 or GDF9. The current commercially available antibodies have been tested in both the Gilchrist (University of Adelaide) laboratory and McNatty (VUW) laboratory and these have been shown to lack specificity and/or unable to recognise recombinant forms of GDF9 or BMP15 standards as assessed by Western blotting.

The monoclonal antibody 3A/A (Mab3A/A) (kindly supplied by Prof. N. Groome, Oxford Brooks University, UK) was raised against the c-terminus end of human BMP15 (K. P. McNatty, personal communication). This sequence shares $83 \%$ homology with rat BMP15. The amino acid sequences are VPYKYVPISVLMIEANGSILYKEYEGMIA (human BMP15) and VPYKFLPMSILLIEANGSILYKEYEGMIA (rat BMP15); the amino acid differences are indicated in bold. Mab3A/A has routinely been used in our laboratory to detect mouse BMP15, which shares 93\% homology with rat BMP15. The mouse sequence is VPYNFLPMSILLIETNGSILYKEYEGMIA; the amino acids that differ to the rat sequence are indicated in bold. Mab3A/A was not used in the Chapter 5 immuno-neutralisation assays because only limited amounts of this material were available and its immuno-neutralising activity had not previously been tested in the rat. However, because of its known ability to recognise mouse BMP15 and that the 93\% sequence homology between mouse and rat BMP15, some studies with Mab3A/A were undertaken for comparison with Mab61A. This was to assist in determining the specificity of Mab61A in recognising rat BMP15. 


\subsection{Methodology}

100 oocytes from rat and sheep were each washed twice in M199 media before incubation in $50 \mu \mathrm{l}$ M199 for $24 \mathrm{~h}$. After incubation, the medium was recovered and the remaining oocytes devoid of spent medium were re-suspended in $50 \mu$ fresh M199. Protease enzyme inhibitors were added to both the medium containing the oocytes and also the spent medium and stored at $-20^{\circ} \mathrm{C}$. Stored samples were only thawed and mixed immediately before the Western blotting procedures to test for the presence of GDF9 and BMP15 proteins.

To test for specificity of the antibodies used, a pre-absorbed antibody aliquot was prepared by incubating the antibody with its target antigen the night before the Western blotting procedure. The Western blots used for the depletion studies were those previously used for identifying the forms of GDF9 or BMP15. These were stripped of their previous antibodies, and Western blotting was performed as before (see Chapter 2.6) but with the pre-absorbed antibody aliquot. After each experiment using pre-absorbed antibodies, the blot was again probed with the appropriate antibodies to confirm that the antigens were still present on the blot (data not shown).

\subsection{Results}

Two attempts were made to identify GDF9 in rat oocyte lysate and spent media (see Figure 6A-D). In the first attempt (see Figure 6A), a mature monomeric rat GDF9 band $(\sim 19-22 \mathrm{kDa})$ was observed in the oocyte lysate with trace amounts of similarsized material detected in the spent media (Figure 6A Lanes 1 and 2). In Figure 6A, Lanes 3 and 4 indicate, BMP15 cross-reactivity (no signal) and the positive control ( $\sim 19-22 \mathrm{kDa})$ respectively. Figure $6 \mathrm{~B}$ shows the corresponding negative control 
Western blot. In this experiment, Mab47B was pre-absorbed with the E. coli produced mature mouse GDF9 protein. The absence of the bands at $19-22 \mathrm{kDa}$ in the oocyte lysate and spent media indicate these bands were Mab47B specific and these sizes correlate with the mature monomeric GDF9 protein. In the second attempt (see Figure 6C), multiple bands were seen in the rat oocyte lysate preparation (Lane 1) but no discernable bands were present in the spent media (Lane 2). In Figure 6C, Lanes 3 and 4 represent BMP15 cross-reactivity and the positive control, respectively. In Lane 1, the multiple bands around $19-22 \mathrm{kDa}$ are probably differently processed forms of the mature monomeric GDF9 protein. The higher molecular bands from $28 \mathrm{kDa}$ to $110 \mathrm{kDa}$ are unknown bands. The bands between $28 \mathrm{kDa}$ and $36 \mathrm{kDa}$ are potentially dimeric forms of rat GDF9, however, as this was done under reducing conditions, this seems unlikely. The $45-54 \mathrm{kDa}$ protein bands are potentially unprocessed pro-mature forms of GDF9. Figure 6D shows the corresponding negative Western blot control. In this experiment, Mab47B was pre-absorbed with the E. coli produced mature mouse GDF9 protein. The evidence from the results in Figure 6D indicate that all but the highest molecular weight forms (i.e. $>54 \mathrm{KDa}$ ) of rat GDF9 could be abolished under the pre-absorption conditions. A mature mouse GDF9 produced by a transfected $293 \mathrm{H}$ cell line was used as a positive control because purified mature rat GDF9 was not available. 

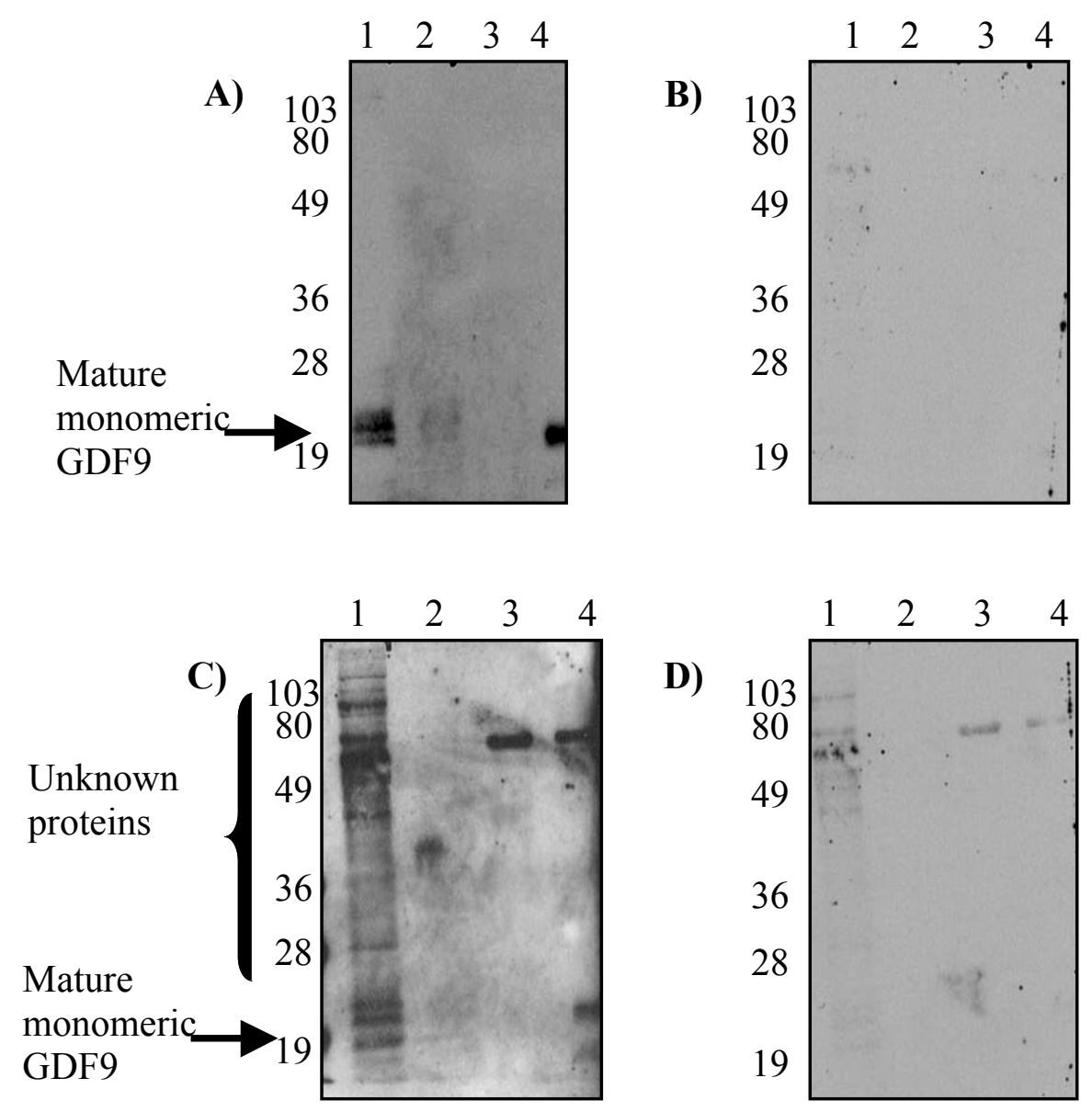

Figure 6A-D: Western blots of GDF9 in rat oocyte lysates and spent media $(\mathrm{A}, \mathrm{C})$ and when Mab47B was pre-absorbed with its specific antigen $(B, D)$. Molecular sizes in $\mathrm{kDa}$ are indicated on the left of each blot. Lanes are as follows, $1=$ =ocyte lysate, $2=$ =spent media, $3=$ transfected $293 \mathrm{H}$ cell produced rat BMP15 for cross reactivity, and $4=$ transfected $293 \mathrm{H}$ cell produced mouse GDF9 positive control.

In the sheep, Figure 6E shows Mab37A detected major protein bands at 14, 21 and $25 \mathrm{kDa}$ in the oocyte lysate (see Lane 1). In Figure 6E Lane 2, bands were also detected at 21 and $25 \mathrm{kDa}$ in the oocyte spent media. These bands are most likely to be mature GDF9 proteins. The multiple bands are probably different processed forms of mature monomeric GDF9 proteins in sheep oocyte lysate and spent media. Figure $6 \mathrm{~F}$ is the corresponding blot for sheep when Mab37A was pre-absorbed with E. coli produced mature sheep GDF9 protein. The mature monomeric GDF9 protein bands 
were absent in Figure 6F, indicating they were Mab37A specific. In Figure 6E and Figure 6F, Lanes 3 and 4 represent the positive control and BMP15 cross-reactivity, respectively. The positive control was sheep GDF9 protein produced by a transfected $293 \mathrm{H}$ cell line. The multiple bands ranging from $\sim 19-25 \mathrm{kDa}$ in Lane 3 are the mature sheep GDF9 positive controls. In Lane 3, the band at $\sim 43 \mathrm{kDa}$ could be the pro-mature sheep GDF9 protein however it is unlikely as it seems too small. The band at $66 \mathrm{kDa}$ is present in both Lanes 1 and 3. Although this band is abolished under the pre-absorbed conditions its size indicates it is likely to be a multimeric form of BMP15, however since this is a performed under reduced conditions this seems unlikely. The presence of the bands in Lane 3 was due to imperfect stripping of the antibodies before Western blotting was performed with the pre-absorbed antibodies. The same Western blot was immuno-stained with a monoclonal antibody raised against sheep BMPRIB, which is not present in the samples. However, the Western blot also showed the same bands in Lane 3 as in Figure 6F (data not shown).
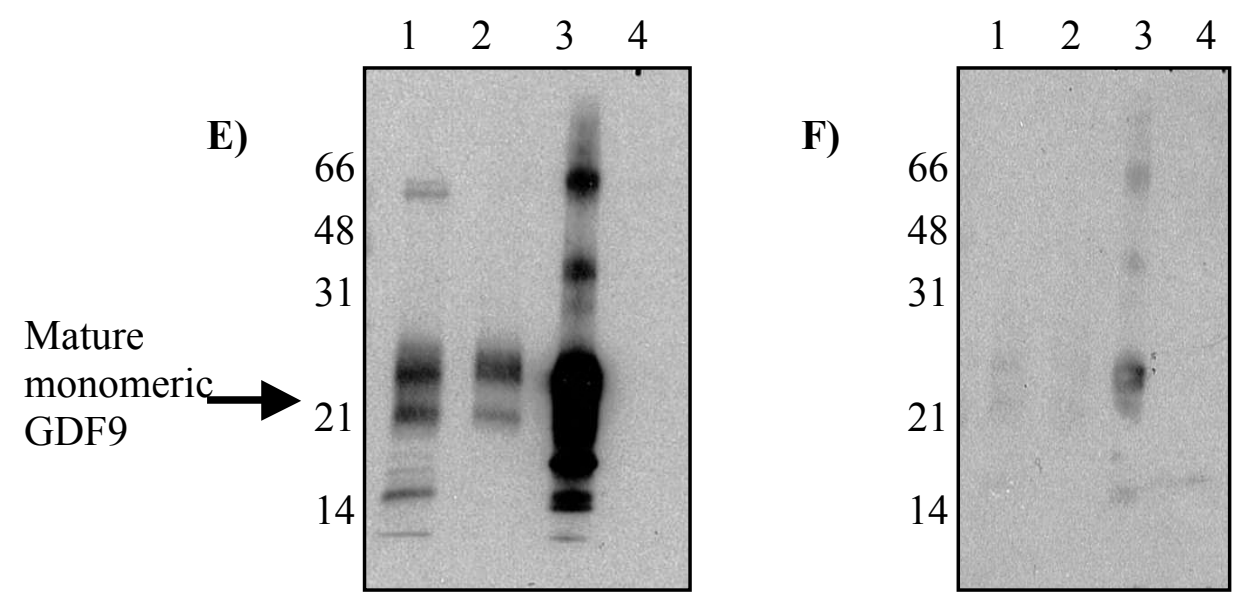

Figure 6E and F: Western blots of GDF9 in sheep oocyte lysates and spent media (E) and when Mab37A was pre-absorbed with its specific antigen (F). Molecular sizes in kDa are indicated on the left of each blot. Lanes are as follows, $1=$ oocyte lysate, $2=$ spent media, $3=$ transfected $293 \mathrm{H}$ cell produced sheep GDF9 positive control, and $4=$ transfected $293 \mathrm{H}$ cell produced sheep BMP15 for cross-reactivity. 
The monoclonal antibody Mab61A was raised against an E. coli produced sheep BMP15. In Figure $6 \mathrm{G}$, Lanes 1 and 2 show that a band at $\sim 17 \mathrm{kDa}$ was present in sheep oocyte lysate and in the spent media. This is most likely the mature monomeric sheep BMP15 protein. In the oocyte lysate there was another band at $\sim 52 \mathrm{kDa}$, which is likely to be pro-mature sheep BMP15 (see Figure 6G, Lane 1). Lanes 3 and 4 represent the positive control and GDF9 cross-reactivity, respectively. The positive control was recombinant sheep BMP15 produced by a transfected $293 \mathrm{H}$ cell line. Figure $6 \mathrm{H}$ is the corresponding Western blot when Mab61A was pre-absorbed with $E$. coli produced mature sheep BMP15 protein. A non-specific band at $14 \mathrm{kDa}$ can be seen in Lane 4.

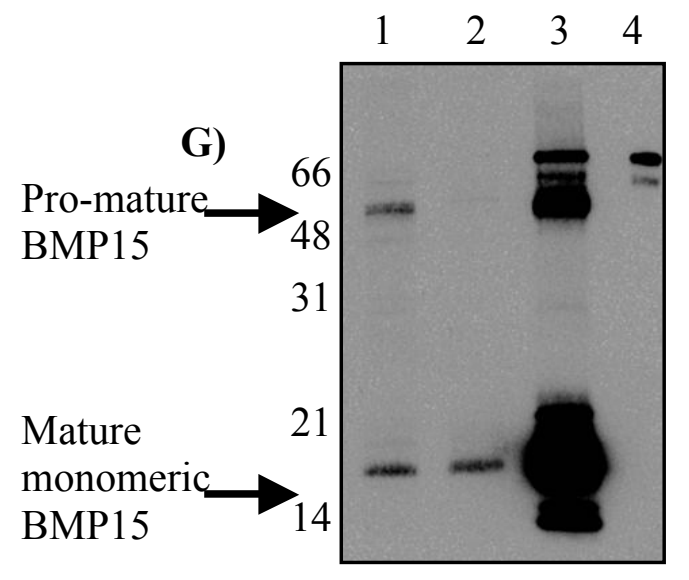

H)

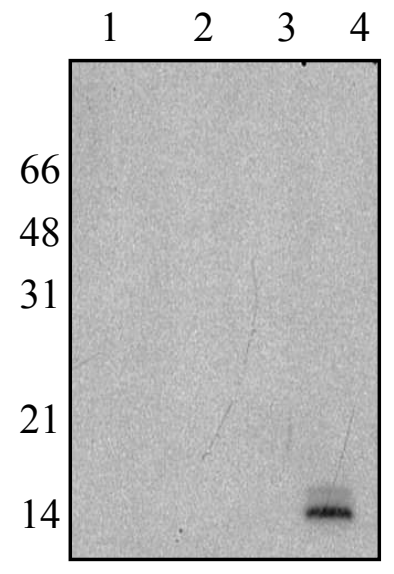

Figure 6G and H: Western blot of sheep BMP15 (G) and the same blot when Mab61A was preabsorbed with its antigen, $E$. coli produced sheep BMP15 (H). Molecular sizes in kDa are indicated on the left of the blot. Lanes are as follows, 1=oocyte lysate, $2=$ spent media, 3= transfected $293 \mathrm{H}$ cell produced sheep BMP15 positive control, and $4=$ transfected $293 \mathrm{H}$ cell produced sheep GDF9 for cross-reactivity.

Figure 6I, Lane 1 shows Mab61A recognised a band just below 49kDa in the concentrated rat oocyte lysate sample (400 oocytes). An oocyte spent media was not included as it was not practical for such a highly concentrated sample. The positive control for this was transfected $293 \mathrm{H}$ cell line produced sheep BMP15, which shows a band just below 49kDa and another band just below 19kDa (see Figure 6I, Lane 4). 
The bigger band at just below $49 \mathrm{kDa}$ is likely to be the pro-mature BMP15 protein and the smaller band at just below $19 \mathrm{kDa}$ is the mature monomeric BMP15 protein. In Figure 6I, Lane 2, a pro-mature band at $49 \mathrm{kDa}$ seems to be present in a concentrated sample from transfected $293 \mathrm{H}$ cell produced rat BMP15 media, but is difficult to distinguish from a non-specific band between 49-80kDa. However, Mab61A did not detect any mature bands in Lane 2.
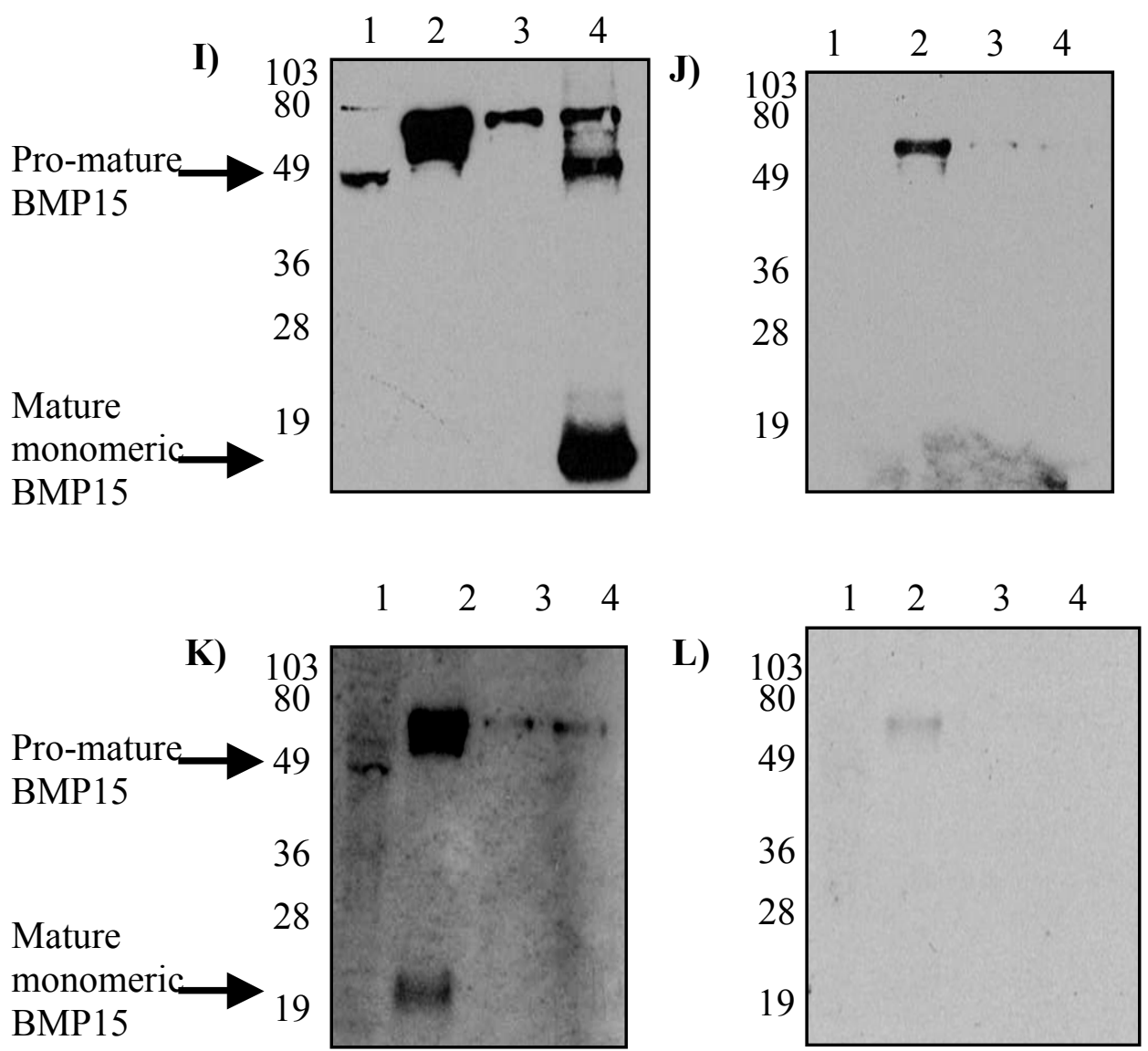

Figure 6I-L: Western blot of rat BMP15 using antibodies Mab61A (I) and Mab3A/A (K), and the same Western blots when the antibodies were pre-absorbed with their specific antigens, $E$. coli produced sheep BMP15 and mouse BMP15 for Mab61A (J) and Mab3A/A (L) respectively. Molecular sizes in kDa are indicated on the left of each blot. Lanes are as follows, $1=$ concentrated oocyte lysate, $2=$ concentrated transfected $293 \mathrm{H}$ cell produced rat BMP15 positive control, $3=$ transfected $293 \mathrm{H}$ cell produced mouse GDF9 cross-reactivity control, $4=$ transfected $293 \mathrm{H}$ cell produced sheep BMP15 positive control.

The same Western blot was re-probed with Mab3A/A. The positive control was the concentrated rat BMP15 media from transfected 293H cell lines (Lane 2) since Mab3A/A did not recognize sheep BMP15 produced by the transfected $293 \mathrm{H}$ cell line 
(Lane 4). In Figure 6K, Lane 1 shows Mab3A/A recognised a band just below 49kDa in the concentrated rat oocyte lysate sample (400 oocytes), and trace amounts of a mature BMP15 band at 19kDa. In Lane 2, which was the rat BMP15 positive control produced by the transfected $293 \mathrm{H}$ cell line, Mab3A/A detected a band at $19 \mathrm{kDa}$. Thus Mab3A/A recognised a mature rat BMP15 band in the $293 \mathrm{H}$ control sample $(19 \mathrm{kDa}$, in Lane 2), and only a trace amount of the mature band at 400 oocytes that was almost undetectable. The band $\sim 78 \mathrm{kDa}$ is most likely a non-specific band.

Figure 6J and Figure 6L show the specificities of the antibodies Mab61A and Mab3A/A, respectively, when Western blotting was performed following preabsorption with their appropriate antigens. The antigen incubated with Mab61A was E. coli produced sheep BMP15, and E. coli produced mouse BMP15 was incubated with Mab3A/A. The only band visible was the non-specific BSA band between 49 and $80 \mathrm{kDa}$. In Lane 3 from Figures 6I-L, there were no cross-reactivity with mouse GDF9 produced by a transfected $293 \mathrm{H}$ cell line, with the exception of the non-specific high molecular band $\sim 78 \mathrm{kDa}$.

\subsection{Conclusion}

The results from the Western blots established that GDF9 mature protein was produced by both rat and sheep oocytes. In addition, the mature GDF9 proteins were also found in the media, albeit at very low concentrations in one of two experiments in the rat. These findings indicate they were secreted from oocytes during the incubation period. At least some of the processed mature forms of GDF9 were biologically active. This interpretation is consistent with the results in Chapter 5 where it was shown that ${ }^{3} \mathrm{H}$-thymidine incorporation by GC was inhibited when 
GDF9 protein was blocked by the addition of GDF9 antibody. The multiple monomeric GDF9 bands for the sheep (see Figure 6E) were most likely the result of differing processing of the mature protein, such as phosphorylation and/or glycosylation (Shimasaki, Moore et al. 2004; McMahon, Sharma et al. 2008). This may be the reason for the multiple bands seen in the rat Western blot (see Figure 6C), however, the multiple bands could also be an artefact of this procedure since the same Western blot had undergone repeated stripping and re-probing with antibodies.

Western blots of sheep samples indicate that sheep BMP15 was produced in the oocyte and was also secreted into media. The oocyte produced forms of BMP15 were biologically active as addition of Mab61A BMP15 antibodies neutralised the stimulatory effect of oocyte-derived BMP 15 on ${ }^{3} \mathrm{H}$-thymidine incorporation by sheep GC (see Chapter 5).

However, rat BMP15 proved difficult to detect. Initially, the sample concentrations

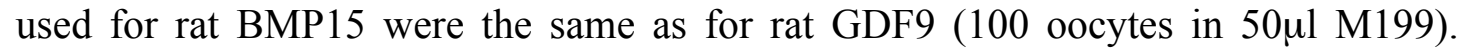
However, despite repeated efforts and positive results for the controls, no visible bands were seen in the oocyte lysate or spent media. The positive controls, using transfected $293 \mathrm{H}$ cell line produced rat BMP15 and sheep BMP15, were always included in the Western blots. They are an important indicator of whether the Western blotting process had worked. In the repeated experiments, the positive controls worked but no mature rat BMP15 protein was seen in the oocyte lysate or spent media samples. 
The concentration of the oocyte lysate was then increased from $\sim 100$ to $\sim 400$ rat oocytes. When Mab61A was used, a visible band at $49 \mathrm{kDa}$ was detected in the concentrated oocyte lysate, and became undetectable when a pre-absorbed aliquot of Mab61A was used. Thus, it is believed that this band represents a pro-mature form of rat BMP15 (Figure 6I). However no mature rat BMP15 bands were seen. To confirm this was not an artefact of the experimental procedure, another antibody to BMP15 (Mab3A/A) was used (Figure 6K). The same Western blot using Mab3A/A showed only a trace amount of mature BMP15 in the oocyte lysate sample. In view of the large number of oocytes required (400) to detect a trace amount of mature BMP15 in the rat oocyte lysate, it supports the idea that little mature BMP15 is present in rat oocytes.

The initial question for this set of experiments was "what were the molecular forms of GDF9 and BMP15 in oocytes during incubation?' To this end, the Western blotting results, under reducing conditions, established that in rats and sheep, oocytes produce GDF9 mature proteins, which were also secreted into the surrounding media. In sheep, BMP15 mature protein was also confirmed to be present in oocyte lysates and spent media. However it seems that rat oocytes synthesize and secrete relatively low levels of BMP15 proteins as these were not detectable in rat oocyte lysates or spent media in a sample of 100 rat oocytes. Pro-mature BMP15 was only detected in rat oocyte lysates when $\sim 400$ oocytes were pooled, and even then only trace amounts of mature BMP15 were observed (Lane 1, Figure 6K). Unfortunately, a Western blot of the rat oocyte spent media sample was not feasible due to a limitation in the amount of media able to be held within a Western blot well. The incubation would have to 
have been at 8 oocytes per $1 \mu$ l. However, it would have been interesting to see if, at this concentration, any rat BMP15 pro-mature protein was present in the medium.

These results provide a new insight into the results for co-incubation of rat DO with either rat or sheep GC in Chapters 4 and 5. The lack of an effect on ${ }^{3} \mathrm{H}$-thymidine incorporation when BMP15 was neutralised in the rat DO-rat GC co-incubations could be because there were no mature BMP15 protein in the media. Also, the lack of rat DO to stimulate ${ }^{3} \mathrm{H}$-thymidine incorporation by sheep GC could also be because sheep GC required a minimum amount of BMP15 mature protein in order to continue proliferation and this amount was not provided when 32 rat DO were present. However, the two response groups seen in Chapter 5 when 64 rat DO were coincubated with sheep GC (3/7 showed sheep GC proliferation, whereas 4/7 did not), could be due to the increased levels of BMP15 when rat DO were concentrated. Keeping in mind that no two oocytes are ever identical, it is possible that some rat DO were expressing and secreting more BMP15. The level of extra BMP15 may have been a small increase, however only a small difference would be sufficient to stimulate sheep GC proliferation, due to the synergistic effects of GDF9 and BMP15 (McNatty, Juengel et al, 2005a; McIntosh, Lun et al. 2008; Reader, Heath et al. 2011). Thus, the Western blot data suggest that the difference between sheep and rats is the relative abundance of BMP15 in DO of rats versus sheep.

The extent of involvement of rat BMP15 pro-mature protein in these experiments is unknown, and further studies to elucidate the potential involvement of BMP15 promature protein in sheep GC ${ }^{3} \mathrm{H}$-thymidine uptake experiments would be very interesting. This is especially so given the recent study by (McIntosh, Lun et al. 2008) 
which points to potential roles of BMP15 pro-region protein in BMP15 and GDF9 interactions in mice. 


\section{$\begin{array}{llllll}\text { Chapter } 7 & \text { EFFECT } & \text { OF } & \text { INCUBATION } & \text { TIME } & \text { ON } \\ \text { GDF9 }\end{array}$ AND/OR BMP15 MRNA EXPRESSION IN OOCYTES}

\subsection{Introduction}

The Western blot results from sheep oocytes indicate that both GDF9 and BMP15 proteins were present in the oocyte lysates and spent media after $24 \mathrm{~h}$. However, in the same number of rat oocytes $(\mathrm{n}=32 /$ well), GDF9 was present in both the lysates and spent media but BMP15 was not detectable. While the presence of the proteins is indicative of biological activity it is not known if the expression levels of GDF9 and BMP15 mRNA change over the incubation period and whether this would have affected the amount of protein produced. Therefore, the aims of the present studies were to investigate whether the levels of $G D F 9$ and/or $B M P 15$ mRNA change over $24 \mathrm{~h}$ in incubation, and whether the change or lack thereof provides further insight into the results from Chapters 4 and 5, where rat DO could not stimulate ${ }^{3} \mathrm{H}$-thymidine incorporation by sheep GC and why rat DO-rat GC co-incubations showed no

significant changes in ${ }^{3} \mathrm{H}$-thymidine incorporation when BMP15 was neutralised (i.e. Chapter 5).

\subsection{Methodology}

See Chapters 2.7-2.11 for the detailed methodology. Briefly groups of $10 \mathrm{COC}$ were collected at intervals of $0,8,16$ or $24 \mathrm{~h}$ after incubation. In addition, co-incubations of 32 DO with 20,000 GC derived from the same species were carried out. Three independent replicate time series experiments were carried out and triplicates were included for each time point resulting in nine replicates for each time point. 
Total RNA for each sample was extracted according to the ArrayPure instructions and the corresponding cDNA synthesised using the Vilo kits from Invitrogen. Negative controls included samples in which water replaced the Superscript III enzyme during cDNA synthesis to test for the absence of genomic DNA. Changes in mRNA levels in samples were quantified by Taqman multiplex QPCR in which GDF9, BMP15 and RPL19 (housekeeper gene) mRNA were measured in the same reaction mix. Each sample was prepared in duplicates and three technical replicates were performed to check for repeatability and technical accuracy. A standard sample of either rat COC or sheep COC was used as calibrator and to standardise for between-assay variances.

The housekeeper gene RPL19 was included in the reaction mix as a reference control. However, when analysing the changes in GDF9 and BMP15 mRNA, the results were not corrected for RPL19. This is because GDF9 and BMP15 are known to be expressed exclusively by oocytes and the number of oocytes in each sample was fixed at 10 in the COC incubations and 32 in the DO incubations (Crawford, Heath et al. 2011). However, RPL19 was an important indicator of the efficacy of the RNA extraction and cDNA synthesis, and template quantity.

From the QPCR measurements, cycle threshold $\left(\mathrm{C}_{\mathrm{T}}\right)$ values were determined and these were normalised with the calibrator value $\left(\Delta \mathrm{C}_{\mathrm{T}}\right)$, and then against the $\Delta \mathrm{C}_{\mathrm{T}}$ values for the control samples $\left(\Delta \Delta \mathrm{C}_{\mathrm{T}}\right)$ within each experiment. The relative linear changes in mRNA levels for each time point were calculated and analysed according to the procedures described in Chapter 2.11. Data that was not normally distributed, as assessed by the Levene's test, was naturally log transformed before analyses. If means 
were significantly different overall then individual means between time intervals were assessed by the Bonferroni post-hoc test. Data that differed by $\mathrm{P}<0.05$ were considered to be significantly different. Ratios between GDF9 and BMP15 mRNA were calculated using the formula, $2^{-(\mathrm{BMP} 15 \mathrm{CT}-\mathrm{GDF} 9 \mathrm{CT})}$. This gives the relative linear fold difference of GDF9 to BMP15 mRNA. Correlation coefficients were calculated as described in Chapter 2.11 .

\subsection{Results}

The reaction efficiencies for the genes RPL19, GDF9, and BMP15 are shown in Table 7A.

Table 7A: Table of QPCR efficiency for each candidate genes.

\begin{tabular}{|c|c|c|}
\cline { 2 - 3 } \multicolumn{1}{c|}{} & \multicolumn{2}{c|}{ Efficiency (\%) } \\
\hline Gene & Rat & Sheep \\
\hline RPL19 & 99 & 93 \\
GDF9 & 99 & 96 \\
BMP15 & 99 & 93 \\
\hline
\end{tabular}

In the rat, mean mRNA expression levels for Rpl19 for all three COC time series experiments are shown in Figure 7A. The second rat time series experiment (TS2) had a very different Rpl19 expression profile compared to TS1 and TS3, and was thus excluded from further analysis. At $16 \mathrm{~h}$ in the rat TS2 experiment, there was only one sample with $\mathrm{C}_{\mathrm{T}}$ values within the sensitive range thus there were no error bars. Mean expression levels of sheep RPL19 for each time series experiment are shown in Figure $7 \mathrm{~B}$. 


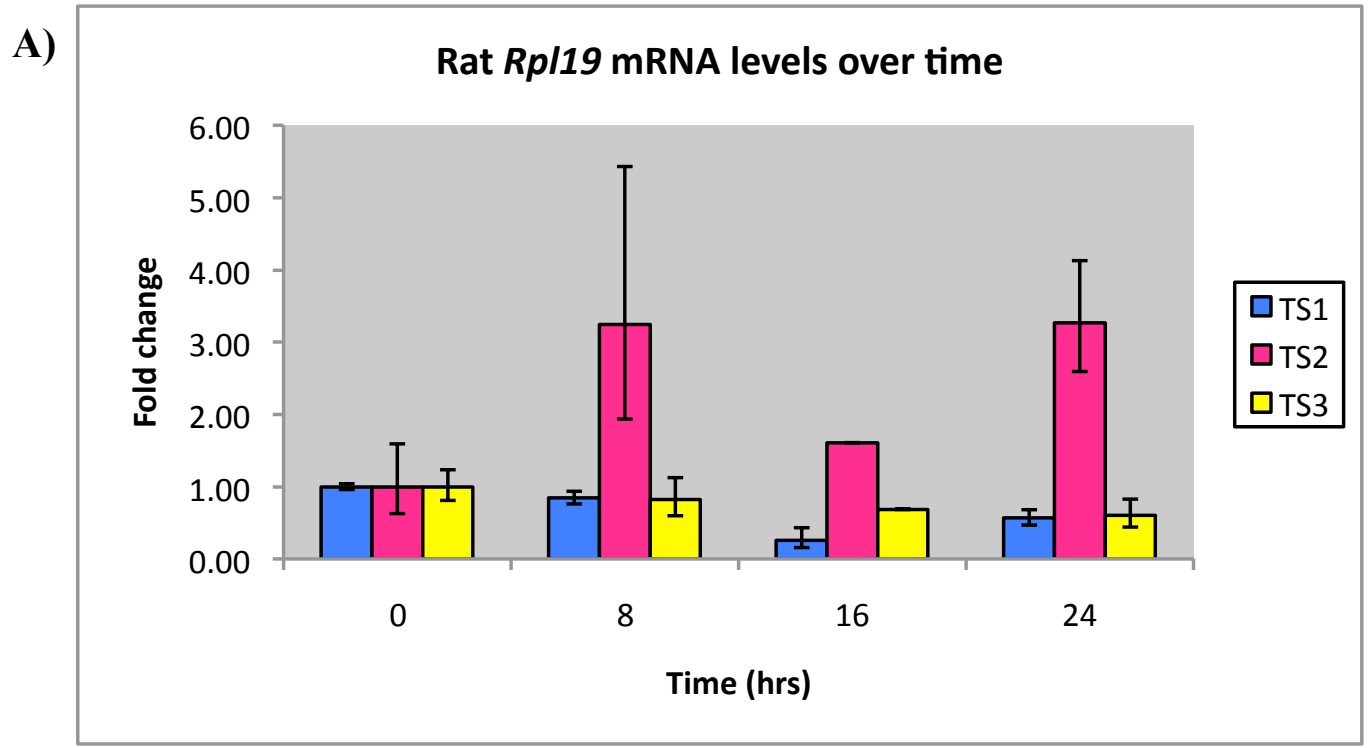

B)

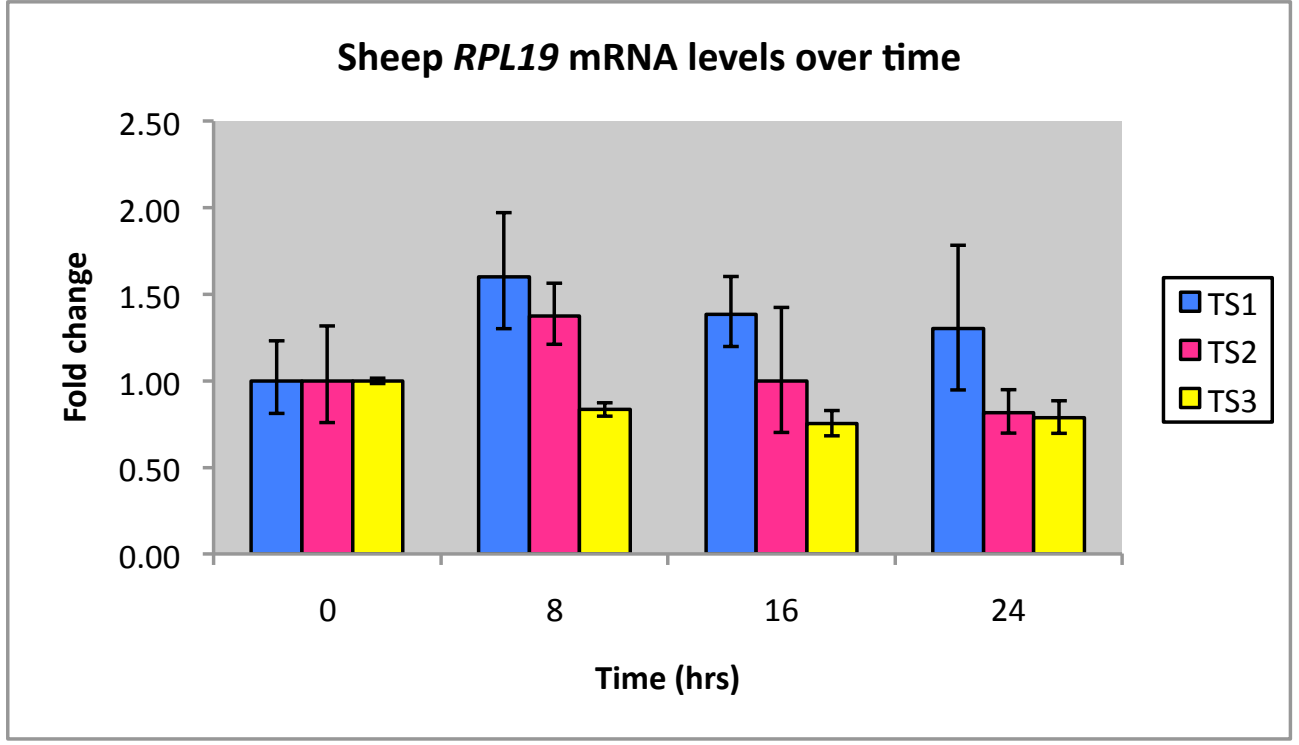

Figure 7A and B: Levels of the housekeeper gene RPL19 mRNA from rat (A) and sheep (B) COC. Columns represent mean mRNA levels from each time series (TS), and the vertical lines represent the upper and lower standard errors of the mean. In 7A, rat TS2 shows a very different expression pattern compared to TS1 or TS3.

The expression levels of GDF9 and BMP15 mRNA from COC in the rat and sheep are shown in Figures 7C and 7D. The pattern of change in expression levels of GDF9 and BMP15 mRNA over time from rat COC and sheep COC showed very strong correlation with each other (Table $7 \mathrm{~B}$ and also Figures $7 \mathrm{G}$ and $\mathrm{H}$ ). There was an overall trend of progressive reduction in GDF9 and BMP15 mRNA expression as incubation time increased in the rat COC and sheep COC incubations. After $24 \mathrm{~h}$ 
incubation, the relative level of rat $G d f 9$ mRNA was significantly lower compared to 0h. Rat Bmp15 showed a significant reduction at both $16 \mathrm{~h}$ and $24 \mathrm{~h}$ compared to $0 \mathrm{~h}$. However, the relative level of rat Bmp15 mRNA at $16 \mathrm{~h}$ was not different to that at $8 \mathrm{~h}$ or at $24 \mathrm{~h}$ (Figure $7 \mathrm{C}$ ). In sheep COC, the relative levels of GDF9 and BMP15 mRNA were significantly lower at $8 \mathrm{~h}, 16 \mathrm{~h}$ and $24 \mathrm{~h}$ compared to $0 \mathrm{~h}$ (Figure $7 \mathrm{D}$ ).

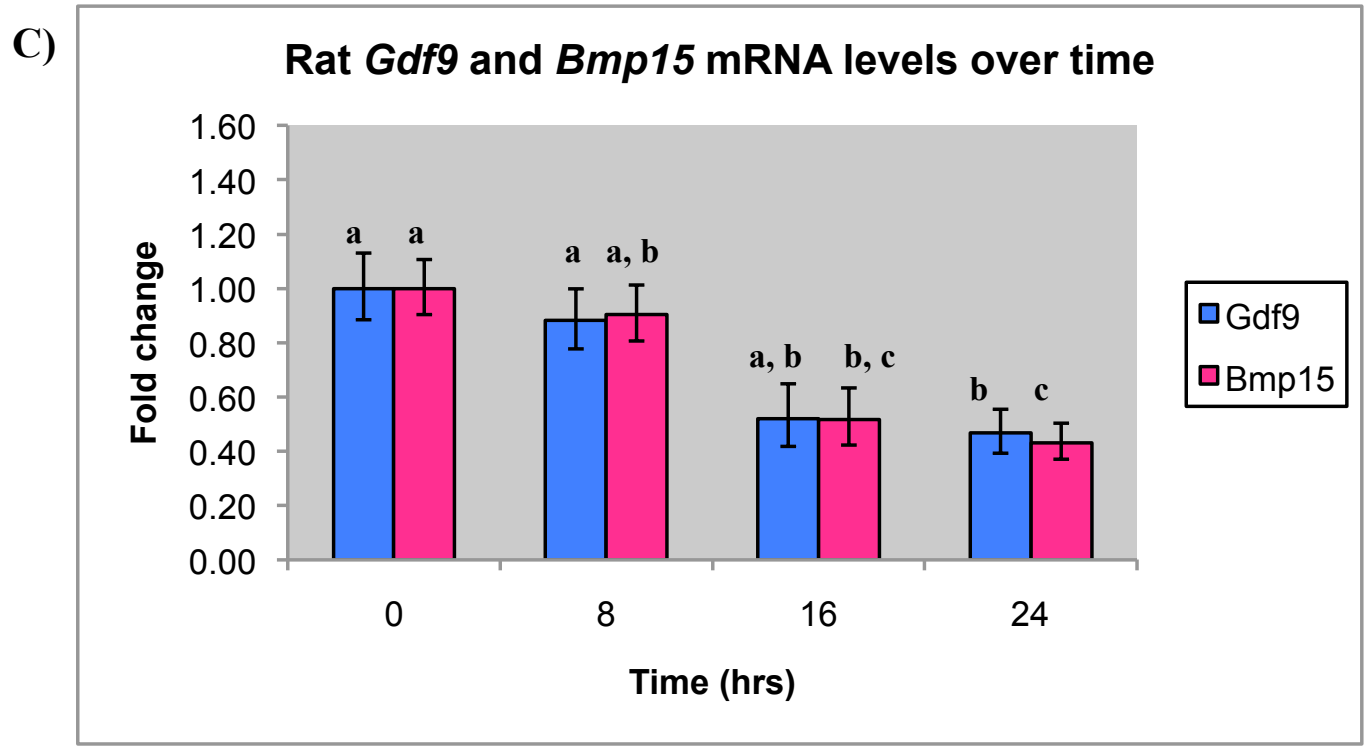

D) Sheep GDF9 and BMP15 mRNA levels over time

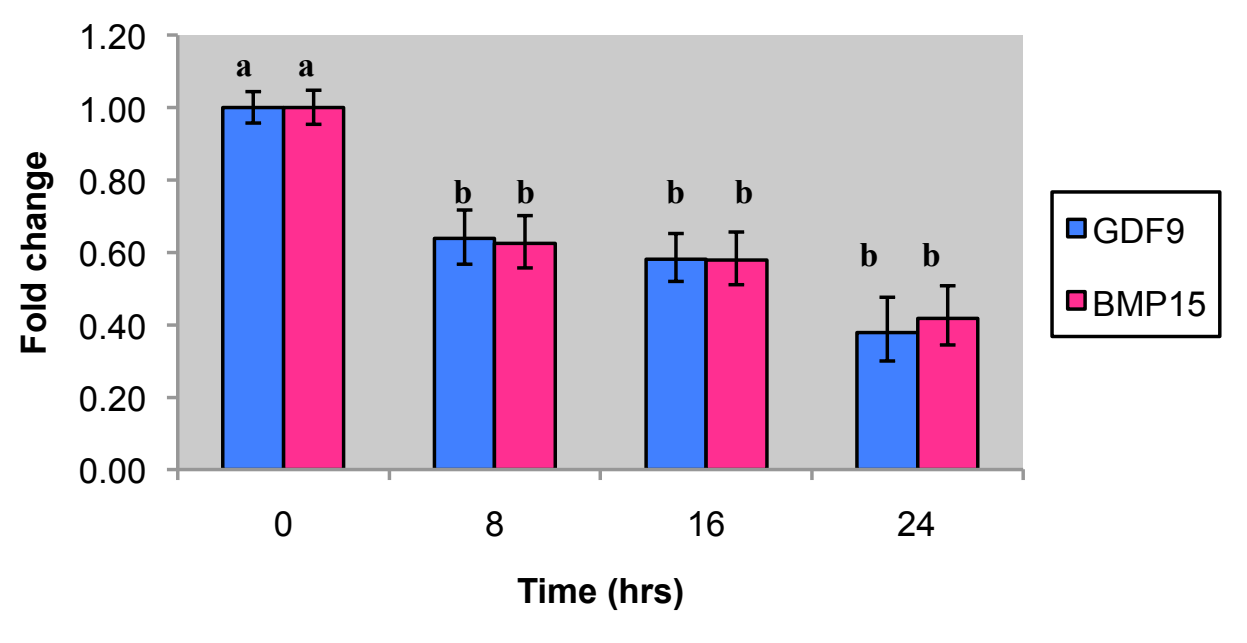

Figure 7C and D: Change in GDF9 and BMP15 mRNA amount in intact COC over time in rat (C) and sheep (D) ( $N=6-9$ individual experiments). Columns represent means and the vertical lines represent the upper and lower standard errors of the mean. For each gene, columns not sharing a common alphabetical letter are significantly different from one another $\mathbf{P}<0.05$. 
In the DO-GC co-incubation experiments, the pattern of change in expression levels of GDF9 and BMP15 mRNA over time also showed very strong correlations with each other, in rats and sheep (Table 7B, and Figures 7G and H). However, in contrast to the findings for the $\mathrm{COC}$, there was no significant change in the pattern of expression levels for either GDF9 or BMP15 mRNA in the DO-GC co-incubations. This is true for both rat and sheep co-incubations (Figure 7E and F). However, a lower overall level of Gdf9 and Bmp15 mRNA can be seen at $24 \mathrm{~h}$ in the rat, although this was not significant. 
E)

\section{Rat Gdf9 and Bmp15 mRNA levels over time}

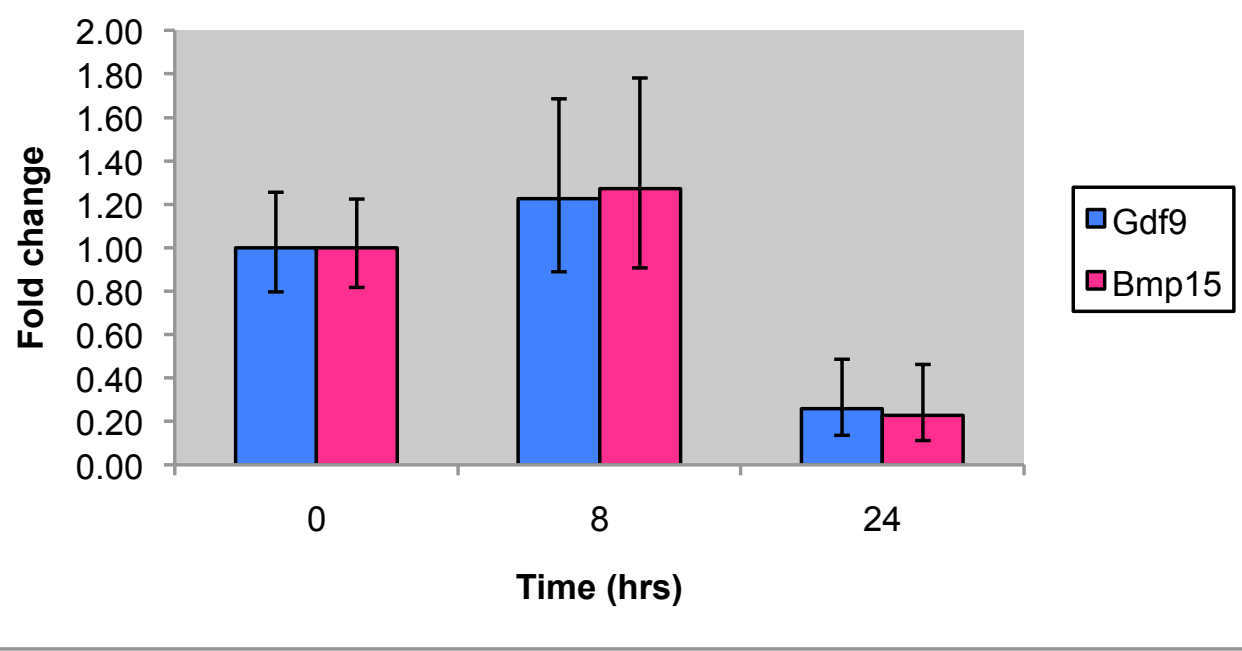

F)

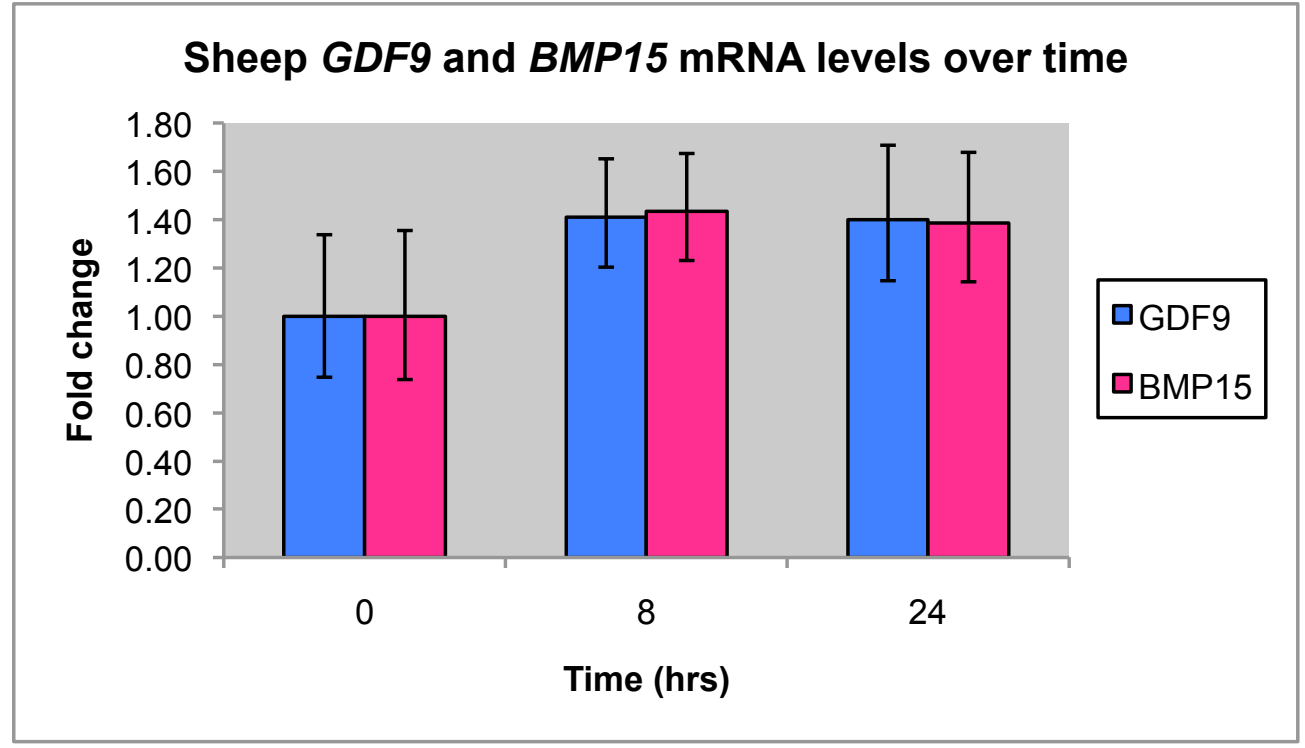

Figure 7E and F: Change in GDF9 and BMP15 mRNA levels in DO over time in rat (E) and sheep (F) $(\mathrm{N}=3$ individual experiments). Columns represent means and the vertical lines represent the upper and lower standard errors of the mean. No statistical differences were noted in $7 \mathbf{E}$ or $7 \mathrm{~F}$.

The ratio of GDF9 to BMP15 mRNA expression levels differed between the two species. In the rat, there was about four times more relative expression of Gdf9 mRNA expression than for Bmp15 mRNA. However in sheep, the ratio of GDF9 mRNA expression levels compared to BMP15 mRNA was 1:1. The ratios of GDF9 to BMP15 mRNA expression levels remained constant over time and were similar in COC and DO co-incubation experiments (Table 7C). 
Table 7B and C: Correlation coefficients between expression levels of GDF9 and BMP15 mRNA in COC and in DO, which were co-incubated with GC for 24h (B). Mean GDF9: BMP15 mRNA expression ratios and the SEM in oocytes incubated as COC or as co-incubations of DO with GC (C)

B)

\begin{tabular}{|c|c|c|c|}
\hline \multicolumn{3}{|c|}{ Correlation coefficients for GDF9 vs BMP15 mRNA } \\
\hline \multicolumn{3}{|c|}{ COC } & \multicolumn{2}{c|}{ DO } \\
\hline Rat & Sheep & Rat & Sheep \\
\hline 0.99 & 0.99 & 0.99 & 0.99 \\
\hline
\end{tabular}

C)

\begin{tabular}{|c|c|c|c|c|c|c|c|c|}
\hline \multirow[b]{4}{*}{ Time (h) } & \multicolumn{8}{|c|}{ GDF9:BMP15 expression ratios } \\
\hline & \multicolumn{4}{|c|}{ COC } & \multicolumn{4}{|c|}{ DO } \\
\hline & \multicolumn{2}{|c|}{ Rat } & \multicolumn{2}{|c|}{ Sheep } & \multicolumn{2}{|c|}{ Rat } & \multicolumn{2}{|c|}{ Sheep } \\
\hline & Mean & SEM & Mean & SEM & Mean & SEM & Mean & SEM \\
\hline 0 & 4.54 & 0.21 & 0.97 & 0.03 & 4.37 & 0.23 & 1.04 & 0.02 \\
\hline 8 & 4.66 & 0.36 & 0.98 & 0.03 & 4.11 & 0.19 & 0.99 & 0.03 \\
\hline 16 & 4.59 & 0.43 & 0.97 & 0.03 & - & - & - & - \\
\hline 24 & 4.87 & 0.08 & 1.04 & 0.02 & 5.08 & 0.65 & 1.05 & 0.02 \\
\hline Average & 4.67 & 0.07 & 0.99 & 0.02 & 4.52 & 0.24 & 1.03 & 0.02 \\
\hline
\end{tabular}


G)

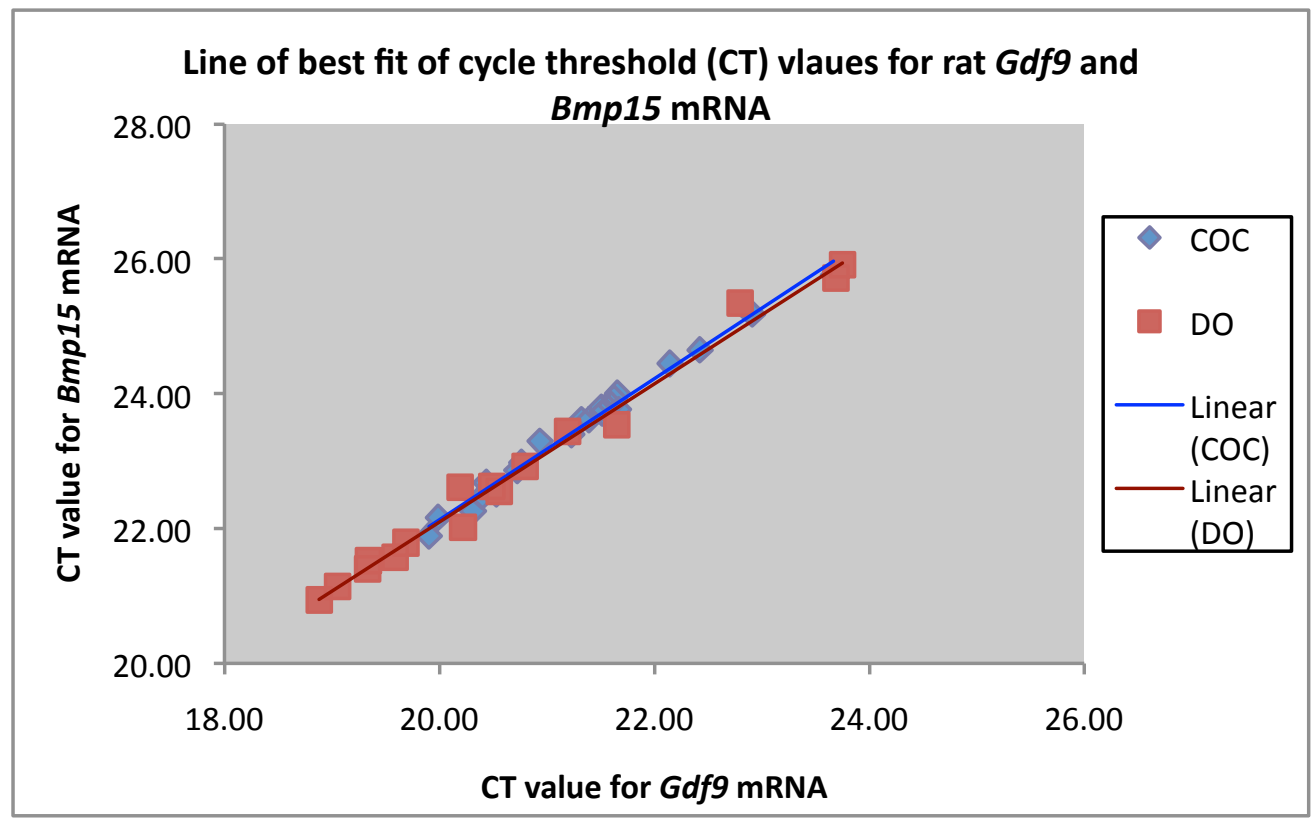

H)

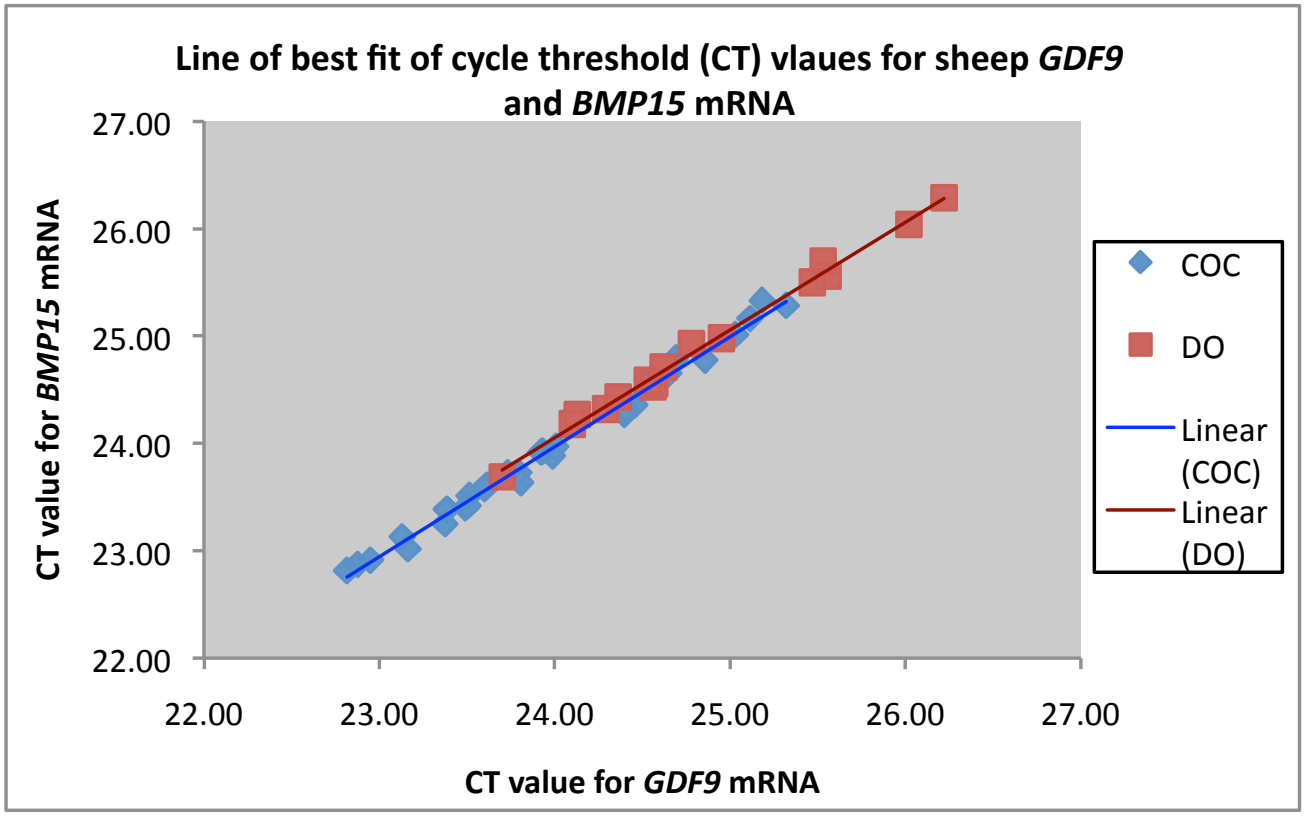

Figure 7G and $\mathrm{H}$ : Regression plots of cycle threshold $\left(\mathrm{C}_{\mathrm{T}}\right)$ values and lines of best fit for BMP15 and GDF9 genes in intact $\mathrm{COC}$ and $\mathrm{DO}$ in rat $(\mathrm{G})$ and sheep $(\mathrm{H})$. The $\mathrm{C}_{\mathrm{T}}$ correlation coefficients for both rat and sheep were 0.99 .

\subsection{Conclusion}

These experiments confirmed that GDF9 and/or BMP15 mRNA expression levels changed in COC with time during a $24 \mathrm{~h}$ incubation period. The expression levels of rat Gdf9 and Bmp15 mRNA in COC showed a significant reduction after 24h and 16h, respectively, when compared to $0 \mathrm{~h}$. In sheep, GDF9 and BMP15 mRNA from COC 
showed significant reductions after $8 \mathrm{~h}$ of incubation as compared to $0 \mathrm{~h}$. In contrast to this, GDF9 and BMP15 mRNA from DO while variable showed no significant changes over time during incubation. In the second time series of the rat $\mathrm{COC}$ experiments, RPL19 mRNA levels indicated a likely experimental error during the culturing or total RNA extraction process. An experimental error during the cDNA synthesis step was also a possibility, however, compared to the other steps it is less likely. Thus experiment TS2 was considered to be an outlier and excluded from further consideration. Therefore the interpretation of Gdf9 and Bmp15 mRNA expression in rat COC over time was based on the two experiments TS1 and TS3.

The reason for the low overall Gdf9 and Bmpl5 mRNA means at $24 \mathrm{~h}$ in the rat DO was due to higher $C_{T}$ values at this time point. The high level of variance was due to lower values in two of the three samples at $24 \mathrm{~h}$, and this large variability could have obscured any significant differences there may be between $24 \mathrm{~h}$ and the other time points. As such, a definite conclusion that Gdf9 and/or Bmp15 mRNA expression levels were maintained or reduced after $24 \mathrm{~h}$ of incubation in the rat DO-GC coincubation will require further studies.

The interesting difference in the relative expression levels of GDF9 and BMP15 mRNA over time between the COC and DO incubations may have been due to differences in the experimental conditions. The intact COC were incubated at 2 $\mathrm{COC} / \mu \mathrm{l}$ in PCR tubes while the DO were incubated at a four-fold lower concentration of $0.5 \mathrm{DO} / \mu \mathrm{l}$ in 96 well plates, identical to that used in the oocyte-GC co-incubation experiments in Chapter 4 and 5. The intact COC experiments were the first to be undertaken. These experiments were undertaken using a higher oocyte to volume ratio 
because the detectable amount of mRNA in single COC was unknown. In addition, the ArrayPure mRNA extraction method did not allow for a large volume of media in a sample. Thus in an attempt to increase the amount of mRNA per sample while keeping the volume of media a minimum, each sample had $10 \mathrm{COC}$ in $5 \mu \mathrm{l}$ total volume. However after these experiments, another set of co-incubation experiments involving DO with GC was performed to replicate the incubation conditions with that of the bioassays in Chapter 4 and 5. However in doing so, the final volume containing the DO-GC co-incubations had to be reduced prior to freezing and storage. While every attempt was made to ensure no DO were lost in this process, some damage could have occurred resulting in rupture of the DO. The difference in procedure thus may have contributed to the discrepancies between the COC and DO-GC incubations experiments. The results from the DO-GC co-incubations are therefore more indicative of changes in oocyte-derived GDF9 and BMP15 mRNA expressions levels that occurred during the ${ }^{3} \mathrm{H}$-thymidine uptake bioassays. These contrasting results indicate how different in vitro conditions can influence experimental outcomes and reinforce the need for caution when translating the findings from different laboratories into physiological processes.

Incubations of oocytes to mimic in vivo situations is an often performed experiment, however, there is very little in current literature regarding the stability or otherwise of GDF9 and BMP15 mRNA levels during time over culture. Most of the studies have focused on GDF9 and BMP15 mRNA levels during oocyte in vitro maturation and in subsequent fertilized embryos (Bebbere, Bogliolo et al. 2008; Li, Kuo et al. 2008; Sánchez, Adriaenssens et al. 2009). The results in the present study show that two of the major growth factors involved in female fertility continue to be expressed through 
the first $24 \mathrm{~h}$ of culture. However, it seems that oocyte function in vitro is likely to be highly sensitive to the incubation conditions as shown by the differences in relative mean expression levels of GDF9 and BMP15 mRNA between COC and DO-GC incubations. Studies with COC from mice or cows show that oocyte quality can be enhanced by the addition of recombinant forms of GDF9 and BMP15 protein, and that GDF9 and BMP15 antagonists reduce oocyte quality (Hussein, Thompson et al. 2006; Yeo, Gilchrist et al. 2008). In the present study, the COC were incubated at $2 \mathrm{COC} / \mu 1$, which may have been too concentrated resulting in rapid depletion of nutrients and change in $\mathrm{pH}$ levels. It is plausible that this in turn lead to poor oocyte health, which was reflected in the results with significant reductions in GDF9 and BMP15 mRNA levels as incubation time increased.

A significant finding was that the ratio of GDF9 to BMP15 mRNA differed between the two species. In rat oocytes, mRNA levels of $G d f 9$ were on average four times more abundant than Bmp15, while in sheep, the ratio was approximately $1: 1$. This result is consistent with the notion that less rat Bmp15 protein is produced compared to Gdf9 whereas in sheep the results are consistent with higher levels of BMP15 protein relative to that in the rat. This further supports the idea that the inability of rat DO to stimulate sheep GC was due to insufficient BMP15 protein produced by rat DO. Therefore in sheep, it is reasonable to speculate that both GDF9 and BMP15 are equally expressed, whereas in the rat $G d f 9$ is the major growth factor for influencing somatic cell function. 


\section{Chapter 8 EFFECT OF TIME IN INCUBATION ON GENE EXPRESSION IN CUMULUS CELLS}

\subsection{Introduction}

Oocytes are reported to have major effects on regulating the phenotype of their adjacent cumulus/granulosa cells (reviewed by Gilchrist, Lane et al. 2008). The objective of these studies was to test the effects of gene expression in CC over the $24 \mathrm{~h}$ interval during the $\mathrm{COC}$ incubation. The candidate genes that were selected for QPCR analyses were FSHR, KITL, AROM, CYCD2, CX43, LHR and the housekeeper gene, RPL19. These genes were selected because they were appropriate markers of GC phenotypes and stages of maturation. Moreover, FSHR and AROM mRNA are expressed exclusively in GC. Correlations between these genes and GDF9 and BMP15 mRNA expression in the COC, as reported in Chapter 7, were also investigated.

FSH is a key hormone required during follicular development and the expression of its receptor, FSHR, is known to be modulated by GDF9 and BMP15 (Vitt, Hayashi et al. 2000; Otsuka, Yamamoto et al. 2001). Mutations in mice have indicated Kit ligand's (KITL) importance in female fertility and studies have shown that its expression in $\mathrm{CC} / \mathrm{GC}$ can also be modulated by GDF9 and BMP15 (Elvin, Yan et al. 1999; Joyce, Pendola et al. 1999; Joyce, Clark et al. 2000; Otsuka and Shimasaki 2002; Thomas, Ethier et al. 2005).

Aromatase gene $(A R O M)$ expression, which is critical for the production of oestradiol by GC, is not maintained when GC are cultured alone. However, studies indicate that 
factors such as FSH and IGF1 maintain or delay the loss of aromatase activity in vitro (Adashi and Hsueh 1982; Picton, Campbell et al. 1999; Marsters, Kendall et al. 2003; Silva, Hamel et al. 2006). However, the role that the oocyte might play in maintaining or influencing aromatase activity in CC in vitro is not known.

Cyclin D2 (CYCD2) is a gene involved in the cell cycle, and thus is likely to influence the mitogenic potential of CC in vitro (Sicinski, Donaher et al. 1996; Chiles 2004; Fuller and Chu 2004). In addition, Connexin 43 (CX43) is a CC/GC expressed protein and part of the gap junction complex providing cell-to-cell communication between the oocyte and CC/GC (Yeager, Unger et al. 1998; Ackert, Gittens et al. 2001). CX43 expression is, in part, indicative of how well cell-to-cell contacts are maintained in COC during incubation. As follicles develop towards ovulation, their growth becomes increasingly dependent on LH (Scaramuzzi, Adams et al. 1993; Messinis, Messini et al. 2010). Thus the $L H R$ gene was also included to see if in COC, the CC maintained low expression levels of $L H R$ or whether increased $L H R$ mRNA was observed over time in incubation. Lastly the housekeeper gene RPL19 was included as a control for RNA extraction and cDNA synthesis quality and template input.

\subsection{Methodology}

The samples used were the same COC samples as described in Chapter 7 (i.e. 10 COC were incubated over $24 \mathrm{~h}$ in $5 \mu \mathrm{l} \mathrm{M199).} \mathrm{As} \mathrm{in} \mathrm{Chapter} \mathrm{7,} \mathrm{rat} \mathrm{time} \mathrm{series} 2$ was taken out of the analyses due to negligible levels of Rpl19 mRNA. In contrast to the Taqman multiplex approach used for the oocyte-related genes, the genes selected to examine $\mathrm{CC}$ function were quantified separately in their own reaction mixes using SYBR Green QPCR methodology. Samples were prepared in duplicate and one to 
four technical replicates were performed for each sample. The level of Rpl19 mRNA was used as a reference for all candidate genes tested.

Relative mRNA levels were calculated as detailed in Chapter 2.11. Resulting linear fold-change values from technical replicates were averaged and a one-way ANOVA was performed. Data that was not normally distributed as assessed by the Levene's test were naturally $\log$ transformed before analysis. If the overall means were significantly different, then individual means between time points were assessed by the Bonferroni post-hoc test. Data sets that differed by $\mathrm{P}<0.05$ were considered to be significantly different.

The mRNA ratios between genes were calculated using the formula, $2^{-(1 \text { st gene CT-2nd gene }}$ CT). This gives the relative fold difference between two genes. Linear regression was used to calculate the correlation coefficient $\left(\mathrm{R}^{2}\right.$ value) and is detailed in Chapter 2.11. See Chapter 2 for the detailed methodology.

\subsection{Results}

The reaction efficiencies for each candidate gene are shown in Table 8A.

Table 8A: Table of QPCR efficiency for each candidate genes.

\begin{tabular}{|c|c|c|}
\cline { 2 - 3 } \multicolumn{1}{c|}{} & \multicolumn{2}{c|}{ Efficiency (\%) } \\
\hline Gene & Rat & Sheep \\
\hline FSHR & 92 & 83 \\
KITL & 90 & 89 \\
CX43 & 93 & 90 \\
AROM & 95 & 90 \\
LHR & 90 & - \\
CYCD2 & 92 & 91 \\
RPL19 & 99 & 93 \\
GDF9 & 99 & 96 \\
BMP15 & 99 & 93 \\
\hline
\end{tabular}


For the rat, relative mRNA levels for $F s h r, C x 43$ and $C y c d 2$ over time in incubation are shown in Figure 8A. For each of these genes, no significant changes in mRNA expression levels were noted over time. The relative mRNA levels for Kitl over time are shown in Figure 8B. Expression levels of Kitl were reduced significantly after $16 \mathrm{~h}$ as compared to $8 \mathrm{~h}$ of incubation, but were not different when compared to $0 \mathrm{~h}$ and $24 \mathrm{~h}$. The relative levels of Arom and Lhr mRNA were below the sensitivity range of the assay at each incubation interval (data not shown). 


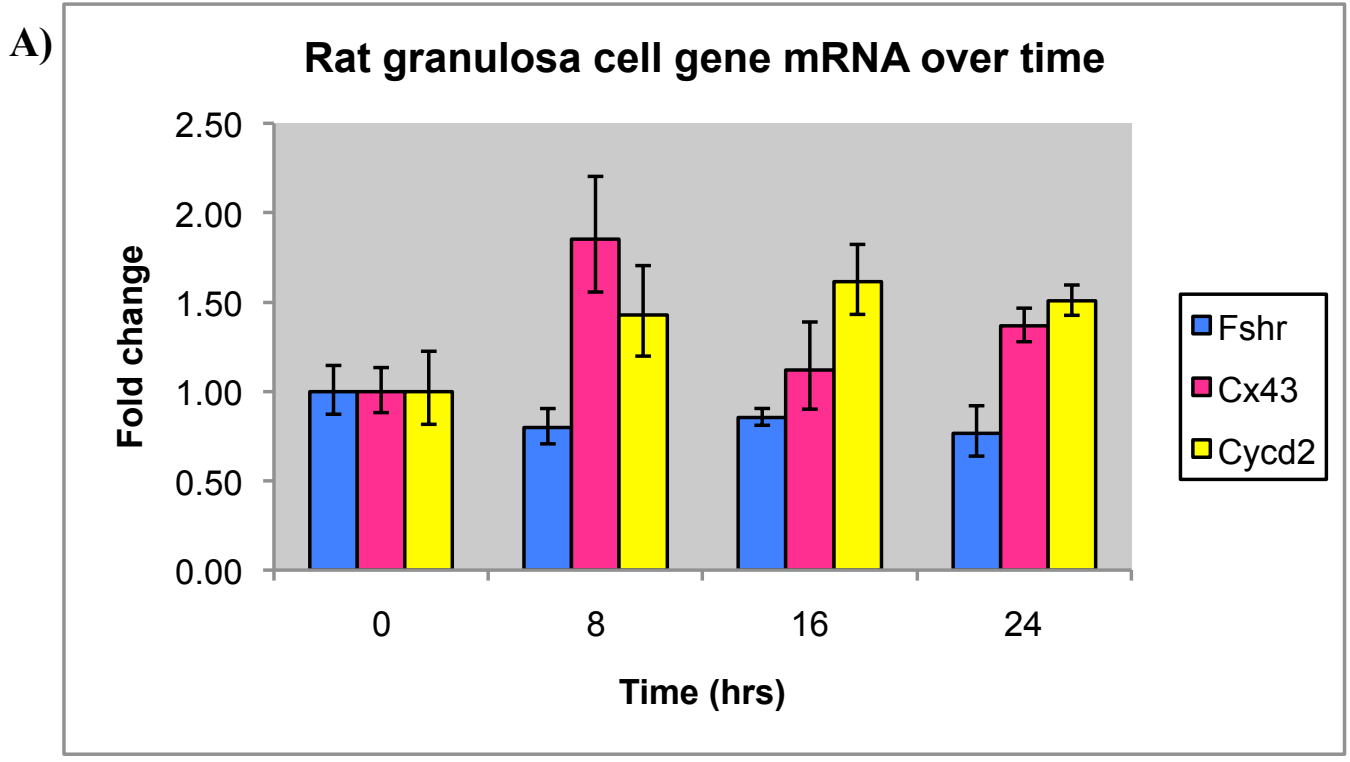

B)

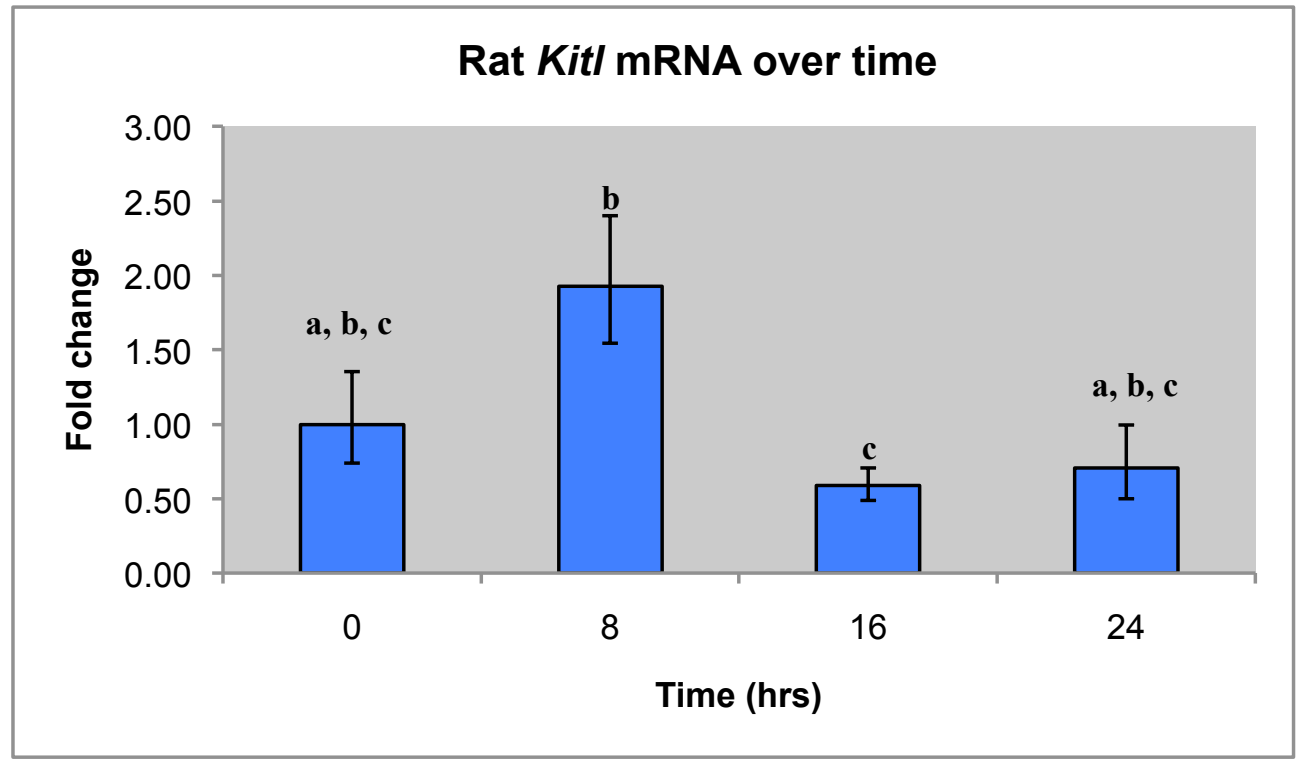

Figure 8A and B: Expression of mRNA levels (A) and specifically of Kitl (B) in rat COC incubated for $24 \mathrm{~h}$. Columns represent means and the vertical lines represent the upper and lower standard errors of the mean. No significant changes in Fshr, Cx43 or Cycd $2 \mathrm{mRNA}$ levels were noted in Figure 8A. In Figure 8B, columns not sharing a common alphabetical letter are significantly different from one another, $\mathrm{P}<0.05$.

In sheep, the relative mRNA levels for FSHR, CX43, KITL or CYCD2 did not change over time during the incubation period (Figure $8 \mathrm{C}$ ). However, the level of $A R O M$ mRNA reduced significantly after $8 \mathrm{~h}$ of incubation as compared to $0 \mathrm{~h}$ (Figure 8D). Thereafter, $A R O M$ mRNA levels declined further at $16 \mathrm{~h}$ and $24 \mathrm{~h}$ of incubation. The 
level of $L H R$ mRNA was below the sensitivity range of the assay at each incubation interval (data not shown).
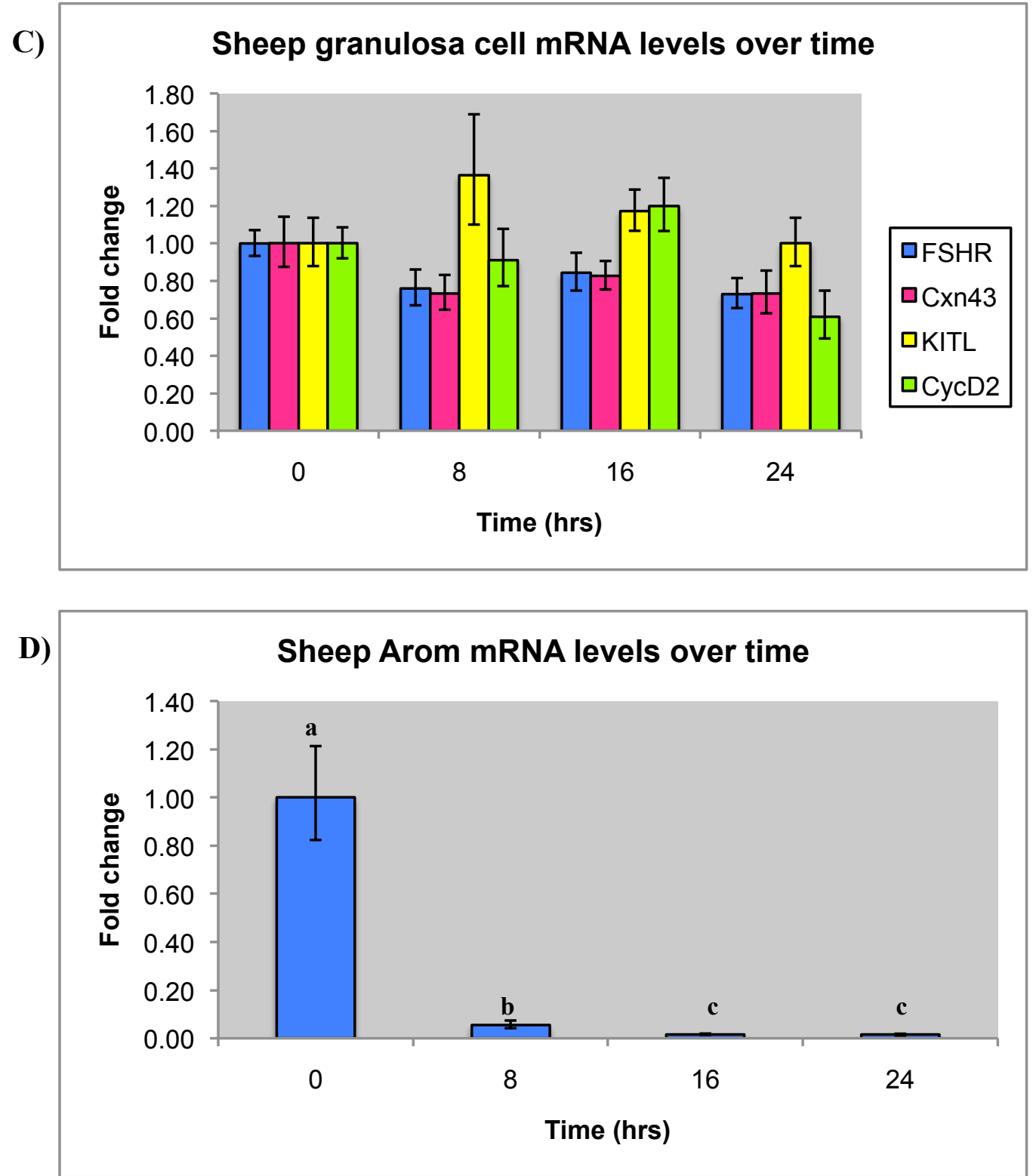

Figure 8C and D: Expression of mRNA levels (C) and specifically of $A R O M$ mRNA (D) in sheep COC incubated for $24 \mathrm{~h}$. Columns represent means as compared to time zero and the vertical lines represent the upper and lower standard errors of the mean. No significant changes in FSHR, CX43, CYCD2 or KITL mRNA levels were noted in Figure 8C. In Figure 8D, columns not sharing a common alphabetical letter are significantly different from one another, $\mathrm{P}<0.001$.

The mRNA expression ratio of each gene compared to every other gene for the rat and sheep COC are shown in Tables 8B and C, and in general did not change significantly 
with increased incubation time. However, in sheep COC, the mRNA expression ratio of $A R O M$ to all other genes changed as time in incubation increased due to a downregulation in $A R O M$ compared to all other genes.

Table 8B and C: Average ratio between genes in rat (B) and sheep (C) COC incubated for $24 \mathrm{~h}$. Each ratio was calculated against the genes indicated along the top row.

B)

\begin{tabular}{|c|c|c|c|}
\hline Gene & Fshr & Kitl & Cx43 \\
\hline Kitl & 0.01 & & \\
Cx43 & 11.00 & 134.00 & \\
Cycd2 & 4.00 & 51.00 & 0.42 \\
\hline
\end{tabular}

C)

\begin{tabular}{|c|c|c|c|}
\hline Gene & FSHR & KITL & CX43 \\
\hline KITL & 0.42 & & \\
CX43 & 1.46 & 7.51 & \\
CYCD2 & 3.99 & 20.97 & 4.00 \\
\hline
\end{tabular}

In the rat, the pattern of change in expression levels of Fshr mRNA correlated strongly with $C x 43, C y c d 2, G d f 9$, and Bmp15 mRNA over time. There were also strong correlations in the pattern of change in expression levels between $C x 43$ mRNA and Cycd2, Gdf9 and Bmp15 mRNA levels. Furthermore, the pattern of change in expression levels of $C y c d 2$ mRNA over time also showed strong correlation with Gdf9 and Bmp15 mRNA levels. However, the pattern of change in expression levels for Kitl mRNA did not correlate with any of the GC genes except for Fshr (Table 8D).

In the sheep, the pattern of change in expression levels of FSHR mRNA over time showed correlation with $C X 43$ and $C Y C D 2$ mRNA, and to a lesser extent with GDF9 and BMP15 mRNA expression levels. Over time, the pattern of change in the expression levels of KITL mRNA showed some correlation with CX43 mRNA. Otherwise the pattern of change in the expression levels of KITL mRNA over time 
showed mild correlation with all other genes. Likewise, the pattern of change in expression levels of $C X 43$ mRNA and CYCD2 mRNA both showed modest correlation with all other genes over time. The pattern of change in expression levels of $A R O M$ mRNA did not show correlation with any of the GC genes over time, and showed a modest correlation with GDF9 and BMP15 mRNA over time (Table 8E).

Table 8D and E: Correlation coefficients for mRNA expression of FSHR, Cx43, CycD2, GDF9 and BMP15 in rat (D) and sheep (E) COC incubated for $24 \mathrm{~h}$. Correlation values are given as $\mathrm{R}^{2}$, and strong correlations (having an $R^{2}$ between $0.6-1.0$ ) are in bold.

D)

\begin{tabular}{|c|c|c|c|c|}
\hline Gene & Fshr & Kitl & Cx43 & Cycd2 \\
\hline Kitl & $\mathbf{0 . 9 7}$ & & & \\
Cx43 & 0.52 & 0.23 & & \\
& & & \\
Cycd2 & $\mathbf{0 . 6 4}$ & 0.15 & $\mathbf{0 . 8 7}$ & \\
Gdf9 & 0.55 & 0.47 & $\mathbf{0 . 6 8}$ & $\mathbf{0 . 6 6}$ \\
Bmp15 & $\mathbf{0 . 6 4}$ & 0.47 & $\mathbf{0 . 6 9}$ & $\mathbf{0 . 6 6}$ \\
\hline
\end{tabular}

E)

\begin{tabular}{|c|c|c|c|c|c|}
\hline Gene & FSHR & KITL & CX43 & AROM & CYCD2 \\
\hline KITL & 0.38 & & & & \\
CX43 & $\mathbf{0 . 6 7}$ & 0.54 & & & \\
AROM & 0.07 & 0.00 & 0.08 & & \\
CYCD2 & $\mathbf{0 . 7 1}$ & 0.39 & 0.34 & 0.02 & \\
GDF9 & 0.40 & 0.27 & 0.37 & 0.34 & 0.23 \\
BMP15 & 0.41 & 0.28 & 0.37 & 0.36 & 0.25 \\
\hline
\end{tabular}

Table 8F and G: Linear regression equations where there was a strong correlation observed for the genes in rats $(F)$ and sheep $(G)$.

F)

\begin{tabular}{|c|l|l|c|}
\hline Gene & \multicolumn{1}{|c|}{ Fshr } & Cx43 & Cycd2 \\
\hline Kitl & $\mathrm{y}=0.4067 \mathrm{x}+16.159$ & & \\
Cycd2 & $\mathrm{y}=1.2616 \mathrm{x}-4.802$ & $\mathrm{y}=0.8171 \mathrm{x}+5.6573$ & \\
Gdf9 & & $\mathrm{y}=0.4392 \mathrm{x}+10.936$ & $\mathrm{y}=0.4657 \mathrm{x}+9.6456$ \\
Bmp15 & $\mathrm{y}=0.7723 \mathrm{x}+5.2963$ & $\mathrm{y}=0.483 \mathrm{x}+12.107$ & $\mathrm{y}=0.5073 \mathrm{x}+10.808$ \\
\hline
\end{tabular}

G)

\begin{tabular}{|c|c|}
\hline Gene & FSHR \\
\cline { 1 - 1 } CX43 & $\mathrm{y}=1.2173 \mathrm{x}-1.7246$ \\
CX43 & $\mathrm{y}=1.2173 \mathrm{x}-1.7246$ \\
\hline
\end{tabular}




\subsection{Conclusion}

These series of experiments were performed to gather information on gene expression changes over time thus relative rather than absolute quantification of mRNA levels were appropriate. The Rpl19 mRNA efficiency was at 99\%, and the other genes were at $83-96 \%$. Thus, it is likely that the values reported for the other genes were underestimated using the $2^{-\Delta \Delta C}$ method as described by Livak and Schmittgen (2001).

In both the rat and sheep, KITL mRNA expression was the lowest followed by FSHR mRNA levels. In the sheep $A R O M$ mRNA was expressed the least. Interestingly, in sheep COC, the expression level of $C Y C D 2$ mRNA was four times more than $C X 43$ mRNA level. In contrast, in the rat Cycd2 mRNA expression level in COC was $\sim 2.4$ times less than Cx43 mRNA. All other genes showed the same ratio abundance in both the rat and sheep. It is possible that the mRNA expression levels of CYCD2 mRNA and FSHR mRNA in GC are influenced by the oocyte growth factors. For example both GDF9 and BMP15 mRNA and protein are expressed by sheep oocytes whereas in the rat, Gdf9 mRNA and protein are detectable, Bmp15 mRNA levels are much lower than the Gdf9 mRNA levels and mature BMP15 protein was not detectable in the spent media.

In the rat, the mRNA levels of Fshr, Cx43, Kitl and Cycd2 in the COC did not change significantly over time. Kitl mRNA did show a significant reduction in mRNA level after $16 \mathrm{~h}$ of incubation when compared to $8 \mathrm{~h}$ but not to any of the other time points. Therefore, this suggests it was likely due to variations inherent in COC and not due to the incubation conditions. In sheep, there were also no significant changes in the 
mRNA levels for any of the genes over time, with the exception of AROM mRNA. The relative levels of $A R O M$ mRNA declined to a low level by $8 \mathrm{~h}$ and were undetectable after $16 \mathrm{~h}$ and $24 \mathrm{~h}$ of incubation. This was not entirely unexpected as AROM mRNA expression has only been able to be maintained when FSH and/or IGF1 have been present in the incubation medium (Adashi and Hsueh 1982; Picton, Campbell et al. 1999; Marsters, Kendall et al. 2003; Silva, Hamel et al. 2006). Therefore, under these incubation conditions, the presence of oocytes alone was unable to maintain $A R O M$ mRNA expression in the incubated CC.

With respect to $L H R$, it can be reasonably expected that little or no $L H R$ were present in the samples from either species. The COC used in the incubations were collected from animals that were mainly pre-pubertal and anovulatory; the lamb ovaries had few if any corpus lutea, and the rats were $21-24 \mathrm{~d}$ old. Gonadotrophin dependence is acquired as the follicle grows towards ovulation (Scaramuzzi, Adams et al. 1993; Dunkel, Tilly et al. 1994; Logan, Juengel et al. 2002). Thus given the age of the animals used, it is not surprising that little to no measurable $L H R$ were found in the COC of rats or sheep.

While these results are interesting, their physiological relevance is uncertain. In vivo, it is known that GDF9 and BMP15 mRNA are maintained throughout follicular development (McGrath, Esquela et al. 1995; Dube, Wang et al. 1998; Laitinen, Vuojolainen et al. 1998; Jaatinen, Laitinen et al. 1999; Bodensteiner, McNatty et al. 2000; Galloway, McNatty et al. 2000). However in the COC incubations, GDF9 and BMP15 mRNA were not maintained in either rat or sheep COC (Chapter 7). In the DO-GC incubation model, the relative levels of GDF9 and BMP15 mRNA were 
maintained over the $24 \mathrm{~h}$ period. In future studies it would be of interest to investigate the mRNA levels of the putative $\mathrm{CC}$ genes in DO-GC co-incubation experimental model. 


\section{Chapter 9 DISCUSSION}

\subsection{Introduction}

Reproductive processes are an inescapable part of nature and life, and there remain many unanswered questions. There is remarkable diversity between mammals in the number of offspring they produce. Animals such as sheep and cow normally produce one or two offspring per birth. However, animals like rats and pigs produce several offspring in a litter. Why is this? Although a simple question, the full answer is likely to be complex and remains to be elucidated.

The simple answer is there must be a difference in the number of oocytes that are released at a given ovulatory cycle: this is commonly referred to as the ovulation quota. In sheep, only one, two or sometimes three oocytes are normally released leading to one or two and occasionally three offspring at lambing. However in rats, 8 to 16 oocytes can be released at ovulation leading to the birth of multiple offspring. Thus, sheep are known as having a low ovulation-rate phenotype and rats as having a high ovulation-rate phenotype.

The question then becomes what regulates ovulation quota in animals with either a low or high ovulation-rate phenotype?

There are several possible molecular candidates, and the two oocyte-derived growth factors GDF9 and BMP15 are the most prominent. They have been shown to have a direct impact on the regulation of oocyte growth and maturation as well as GC proliferation and differentiation. Thus, oocytes have profound effects on 
folliculogenesis in general and potentially a key role in regulating the number of ovulatory follicles that go on to ovulate (Dong, Albertini et al. 1996; Carabatsos, Elvin et al. 1998; Elvin, Clark et al. 1999; Elvin, Yan et al. 1999; Galloway, McNatty et al. 2000; Otsuka, Yao et al. 2000; Hanrahan, Gregan et al. 2004; Juengel and McNatty 2005; McNatty, Juengel et al. 2005a; Edwards, Reader et al. 2008; Huang, Cheung et al. 2009; Nicol, Bishop et al. 2009).

In the present study, the objective was to examine the role of the oocyte in regulating GC proliferation in vitro, and specifically in the actions of GDF9 and/or BMP15 between species such as sheep (a low ovulation-phenotype) and rats (high ovulationphenotype).

\subsection{Oocytes stimulate ${ }^{3} \mathrm{H}$-thymidine incorporation in GC of the same species but not necessarily in GC derived from a different species}

An in vitro model to investigate the short-term effects of oocyte regulation of follicular growth was established by co-incubation of oocytes with GC. The first step was to optimise the co-incubation conditions for the rat and sheep experiments. The level of ${ }^{3} \mathrm{H}$-thymidine incorporation is a measure of DNA synthesis in GC, which is a prerequisite for cell proliferation, and was used in the present study as an indirect measure of GC proliferation (Gilchrist, Morrissey et al. 2003). Based on the results from Chapter 3, oocytes from both rat and sheep were found to stimulate ${ }^{3} \mathrm{H}$ thymidine incorporation by rat and sheep GC respectively, in a dose dependent manner. These studies show that a pool of 32 or more rat oocytes secreted enough stimulating factor(s) over a $24 \mathrm{~h}$ incubation period to reproducibly enhance ${ }^{3} \mathrm{H}$ thymidine uptake by rat GC. In the sheep co-incubations, considerable variability was 
observed in the degree of ${ }^{3} \mathrm{H}$-thymidine incorporation between experiments. Its likely that one explanation of the variability in the ${ }^{3} \mathrm{H}$-thymidine incorporation in the DOGC bioassays was due to altered secretions of either BMP15 or GDF 9 as a result of heterogenous and functionally different populations of DO between replicate experiments. In the McNatty et al. (2005) study, small changes in either BMP15 or GDF9 caused major changes (up to 7 fold) in ${ }^{3} \mathrm{H}$-thymidine incorporation.

Ideally, it would have been preferable to use 64 sheep oocytes in subsequent coincubation experiments but this was not practical on a routine basis when undertaking replicate experiments. However, the results obtained were in accordance with published studies on mice and the cow where oocytes have been shown to modulate GC activity (Li, Norman et al. 2000; Gilchrist, Ritter et al. 2001; Gilchrist, Morrissey et al. 2003; Gilchrist, Ritter et al. 2006; Gilchrist and Thompson 2007; Gilchrist, Lane et al. 2008; Yeo, Gilchrist et al. 2009).

The question could be asked as to why so many oocytes were needed for each in vitro study when physiologically, each follicle contains only one oocyte. In part, it can be justified by the fact that the in vitro experiments involved collecting oocytes and GC from both healthy and potentially atretic follicles over several hours following animal slaughter and ovarian recovery, mechanically 'stripping' the oocytes of CC and then undertaking the experiments in an incubation volume of $55 \mu$. Thus, as a consequence of pooling cells, a mix of oocytes and/or GC of different quality and differentiative states were used. Secondly, the volume of follicular fluid in follicles from which the $\mathrm{COC}$ and GC were recovered would have been significantly less than $55 \mu 1$ and often $<8 \mu 1$. Thus, the physiological concentrations of secreted oocyte growth factors given 
the small fluid volume and presence of a proteoglycan matrix in follicular fluid is likely to be relatively high in vivo. To compensate, the present study included oocytes over a concentration range of between one per $7 \mu 1$ to one per $0.85 \mu 1$. Co-incubations at higher volumes of $125 \mu \mathrm{l}$ were initially tested but were found to be too dilute to achieve stimulation. Routinely, the concentration of oocytes for the key test experiments was at one per $1.7 \mu 1$, which is likely to have supplied oocyte-derived factors in physiological ranges.

To assess for potential species differences in oocyte stimulation of GC proliferation, sheep oocytes were co-incubated with rat GC and vice versa. Thirty-two sheep oocytes were able to stimulate ${ }^{3} \mathrm{H}$-thymidine incorporation in both sheep and rat GC. Significantly however, 32 rat oocytes were not able to stimulate ${ }^{3} \mathrm{H}$-thymidine incorporation by sheep GC (see Chapter 4). On some occasions (i.e. 3/7) 64 but not 32 or fewer rat oocytes significantly stimulated ${ }^{3} \mathrm{H}$-thymidine incorporation.

It is possible that rat oocytes were producing factors detrimental to the proliferation of sheep GC. This has been seen when mouse GDF9 was added to sheep or cow GC in vitro (McNatty, Juengel et al. 2005b). However there have also been many studies that have used recombinant proteins based on a species different to that of the GC or follicles to study its effect (Otsuka, Yao et al. 2000; Kaivo-Oja, Bondestam et al. 2003; Lee, Yoon et al. 2004; Liao, Moore et al. 2004; McNatty, Juengel et al. 2005a; McNatty, Juengel et al. 2005b; McNatty, Lawrence et al. 2006; Sugiura, Su et al. 2007; Mottershead, Pulkki et al. 2008). Alternatively, critical oocyte-derived proteins from rat oocytes such as GDF9 and/or BMP15, may not have been in recognisable forms for sheep GC to utilise and/or they were produced in insufficient amounts. The 
results from Chapters 5 through 7 indicate the latter might have indeed been the case with respect to the oocyte-derived proteins GDF9 and BMP15, especially BMP15.

\subsection{GDF9 and BMP15 are responsible for oocyte stimulatory effects on ${ }^{3} \mathrm{H}$ -} thymidine incorporation in GC

As part of the aims of this study, the relative importance of GDF9 and/or BMP15 in regulating ${ }^{3} \mathrm{H}$-thymidine incorporation in GC was investigated by selectively immunoneutralising these factors through the addition of specific monoclonal antibodies in the oocyte-GC co-incubations.

The addition of the monoclonal GDF9 antibody (Mab47B), resulted in reduced ${ }^{3} \mathrm{H}-$ thymidine incorporation by GC in all species combinations tested, indicating that oocyte-derived GDF9 is a very important factor produced by oocytes for proliferation of GC in both rats and sheep. The fact that a higher concentration of GDF9 antibody (made against the sheep GDF9 c-terminus) was needed to neutralise oocyte-derived GDF9 in rat incubations may indicate more GDF9 mature protein was present in rat bioassays. However, a more likely explanation is that there were different binding affinities of the antibody to rat and sheep GDF9. For example, the antibody concentration may not have been optimal against rat GDF9 mature protein as it was made against a sheep GDF9 mature protein sequence. The GDF9 amino acid sequences in the rat and sheep shared a 93\% homology (Chapter 5), and there was a nine amino acid sequence that was identical between the two species. This level of homology has previously been shown to be sufficient for antibody-antigen recognition for members of the TGF- $\beta$ superfamily (McNatty, Hudson et al. 2007). As there were 
no other bioactive GDF9 antibodies available for comparative studies, this was the best option available at the time the experiments were undertaken.

Neutralising oocyte-derived BMP15 with Mab61A also resulted in a significant reduction in ${ }^{3} \mathrm{H}$-thymidine incorporation by GC in co-incubations of sheep oocytes with either sheep or rat GC. However, it did not result in any significant reduction in ${ }^{3} \mathrm{H}$-thymidine incorporation by rat GC with rat oocytes. This may have been due to at least two reasons namely antibody optimisation and/or antibody recognition.

First, the antibody used was not optimal or did not recognise rat BMP15. Bioactive, rat specific BMP15 antibodies were not commercially available and of the available antibodies, Mab61A was the only antibody with proven bioactivity (McNatty, Juengel et al. 2005b; McNatty, Hudson et al. 2007). An alternate antibody, Mab3A/A, made against human BMP15 was the second option available. However, its ability to neutralise BMP15 in the incubations was uncertain and there were only limited quantities of this antibody available, which prevented extensive testing. The present study established that Mab61A was able to recognise the pro-mature rat BMP15 and this was confirmed by Western blotting with oocyte lysates (Chapter 6). Moreover, the Western blotting studies showed that the binding of Mab61A to rat BMP15 could be blocked using an E. coli-derived mature BMP15.

Secondly, it was possible that the concentration of antibody used was insufficient to show an effect. However, it seems more likely that mature rat BMP15 protein was absent or present at very low concentrations in the media from the rat oocyte-rat GC co-incubations. The lack of mature rat BMP15 protein in the Western blots of a 
concentrated rat oocyte lysate sample suggests that very little, if any BMP15 protein was present.

Collectively, these results indicate that sheep oocytes regulate ${ }^{3} \mathrm{H}$-thymidine incorporation by GC via GDF9 and BMP15, whereas rat oocytes regulate ${ }^{3} \mathrm{H}$ thymidine incorporation by GC via GDF9 but not BMP15 regulation. Whether oocyte-derived growth factors other than BMP15 and/or GDF9 are involved was not tested in the present study. It seems likely that sheep GC require both GDF9 and BMP15, but rat GC requires GDF9 but not BMP15 for proliferation. In future studies when purified recombinant GDF9 and BMP15 proteins become available this notion can be confirmed or refuted by co-incubating DO with GC in dose-related studies with or without purified BMP15.

\subsection{Rat and sheep oocytes show different BMP15 mRNA and protein expression patterns}

The results from the Western blotting experiments showed that GDF9 mature protein was present in both rat and sheep oocyte lysates (Chapter 6). In addition mature GDF9 proteins were also found in the media, indicating they were produced and secreted from oocytes during incubation. These results were not unexpected given the importance of GDF9 in GC cultures as reported in the literature (Elvin, Clark et al. 1999; Otsuka, Yao et al. 2000; Vitt, Hayashi et al. 2000). The multiple sheep GDF9 bands in the Western blot were most likely the result of differing processing of the mature protein, for example glycosylation and/or phosphorylation (Shimasaki, Moore et al. 2004; McMahon, Sharma et al. 2008). 
The most interesting finding from the immuno-neutralisation studies was the evidence for differential effects between rats and sheep based on the role of BMP15. These findings are in accordance with in vivo examples, where naturally-occurring BMP15 inactivating mutations leads to infertility in the sheep, whereas inactivating mutations in mice shows no significant impact on follicular development (Galloway, McNatty et al. 2000; McNatty, Juengel et al. 2001; Yan, Wang et al. 2001; Hanrahan, Gregan et al. 2004).

Rat BMP15 protein proved to be very hard to detect. Initially, the sample concentration used to detect rat BMP15 protein was the same as that used for rat GDF9 protein detection. However, despite repeated efforts, no visible bands were seen in the oocyte lysate or spent media. The concentration of the oocyte lysate was increased from $\sim 50$ to $\sim 400$ rat oocytes to increase protein concentration. One complication may have been that the Mab61A antibody was made against a sheep BMP15 protein. Therefore its ability to recognise rat BMP15 protein may not have been as sensitive and a concentrated sample was needed. When Mab61A was used, a visible and specific band at $49 \mathrm{kDa}$ was detected in the concentrated oocyte lysate and this is considered to be the pro-mature rat BMP15 protein. However no mature protein bands for rat BMP15 were observed (Chapter 6). To confirm the absence of mature rat BMP15 protein, Mab3A/A was used.

The monoclonal antibody Mab3A/A was made against a human BMP15 C-terminal 32 amino acid sequence of the mature protein. This shares an $83 \%$ amino acid sequence homology with rat BMP15 protein (Chapter 6). However, due to limited amounts of this antibody it was only suitable to act as a control for the specificity of 
Mab61A against rat BMP15 protein. The same Western blot using Mab3A/A also showed a lack of mature rat BMP15 protein in the oocyte lysate sample. It also detected the same band at $49 \mathrm{kDa}$ in the concentrated rat oocyte lysate sample confirming the results shown by Mab61A, and indicating that the lack of a mature rat BMP15 protein in the concentrated rat oocyte lysate sample was not a caveat of the Mab61A antibody but a true result. However, Mab3A/A detected mature BMP15 proteins in the transfected $293 \mathrm{H}$ cell produced rat BMP15 positive control.

There are a number of possible explanations for the absence of mature rat BMP15 protein in rat oocyte lysate. The first possible explanation is there were no visible rat BMP15 mature proteins in the oocyte lysates because most of it was secreted into the surrounding media. A corresponding spent media sample for the $\sim 400$ oocyte lysates over $24 \mathrm{~h}$ was not undertaken for practical reasons, as discussed in Chapter 6 , there was a physical limitation on the sample volume able to be loaded within a Western blot well. However if rat BMP15 protein was in fact secreted from oocytes, one would have expected that, at least, some protein would have been observed in the spent media. As there was no indication of this, it seems that very little of the pro-mature form is processed by the oocytes into mature forms for secretion into the media. However, it would be interesting to confirm this with alternative species-specific antibodies in the future. A second possibility is that most, if not all of the secreted mature BMP15 proteins were rapidly degraded. If this was the case then the oocyte lysate at the point of collection should have some detectable mature protein. The concentrated rat oocyte lysate sample was collected and frozen immediately and no detectable amount of mature BMP15 protein was evident. Thus, it seems unlikely that rapid degradation was a major contributing factor, as it did not have time to degrade. 
A third possibility was that the Western blotting method might not have been sensitive enough to detect any mature BMP15 proteins that were present. One of the major drawbacks of Mab61A is that it was made against sheep BMP15 and the indications were that it might have had some difficulty in recognising mature rat BMP15. However, when Mab3A/A was used little mature rat BMP15 protein in the rat oocyte lysate sample was detected, so it is unlikely that a lack of sensitivity of Mab61A was the issue. Nevertheless, a lack of the sensitivity in the Western blotting procedures cannot be completely ruled out as a contributing factor. Lastly, the absence of mature rat BMP15 may be a genuine difference in oocyte expression and processing between rats and sheep. The literature for BMP15 in rodents is conflicting. Jaatinen, Laitinen et al. (1999) and Dube, Wang et al. (1998) reported that BMP15 protein was present from the pre-antral stages in rats and mice. However, Yoshino, McMahon et al. (2006) found in mice that mature BMP15 protein was only detectable just before ovulation. Studies by Yan, Wang et al. (2001) did however support the notion that rodent BMP15 is more important during the final stages of follicular development than earlier. These authors found that ovaries from BMP15 null mice were virtually indistinguishable from the wild type suggesting that follicular development was not perturbed in the absence of BMP15 protein. However null mice showed consistently reduced litter sizes. This was due to difficulty in releasing oocytes from follicles and to less fertilizable oocytes (Yan, Wang et al. 2001). Observations from these and other studies in rodents indicate that BMP15 is not essential for follicular growth or ovulation but of some importance for all the ovulating oocytes to reach their full developmental competencies (Gueripel, Brun et al. 2006; McMahon, Hashimoto et al. 2008). Thus, the most plausible interpretation from these studies is that rat BMP15 protein secretion is very low before the pre-ovulatory LH surge. The lack of mature 
BMP15 protein in rat oocytes in the present study was likely to be due to the fact that the ovaries were recovered from rats at the late pre-pubertal period and few if any preovulatory follicles would have been included. In contrast, studies in sheep have found mature BMP15 protein from primary follicles onwards, and homozygous BMP15 inactivating mutations or immunisation of BMP15 in sheep result in infertility due to an arrest of normal follicular development from the primary stage (Galloway, McNatty et al. 2000; Juengel, Hudson et al. 2002; Juengel, Hudson et al. 2004; McNatty, Hudson et al. 2007). Thus it is reasonable to conclude from this and related published studies that there is a clear species difference in the degree of involvement of BMP15 in folliculogenesis between rats and sheep.

The differences discussed above strongly point to BMP15 as a major contributing factor in the lack of ${ }^{3} \mathrm{H}$-thymidine incorporation in co-incubations of rat oocytes with sheep GC. Sheep GC required both BMP15 and GDF9 protein to proliferate and thus incorporate ${ }^{3}$ H-thymdine. Both GDF9 and BMP15 protein were produced by sheep oocytes, suggesting that they stimulated ${ }^{3} \mathrm{H}$-thymidine incorporation in sheep GC. However when sheep oocytes were substituted by rat oocytes, there was a lack of mature BMP15 protein in the co-incubation and the result was as if BMP15 was neutralised leading to a significant reduction in ${ }^{3} \mathrm{H}$-thymidine incorporation by sheep GC. The existence of the two distinct responses in the rat oocytes with sheep GC coincubations in Chapter 4 indicate that in some instances when very large numbers of rat oocytes, (e.g. 64 oocytes) were used, the accumulated level of mature BMP15 protein from all the rat oocytes in the incubation provided a sufficient amount to stimulate ${ }^{3} \mathrm{H}$-thymidine incorporation by sheep GC. There were no observable 
differences in rat oocytes or sheep GC between those that responded and nonresponders to 64 rat oocytes co-incubated with sheep GC.

An alternative, although less likely, scenario is that mature rat BMP15 protein was produced but folded in such a way that the antibodies used were unable to recognise the protein. Thus, it cannot be completely ruled out that rat BMP15 protein may have been present in the rat oocyte-sheep GC co-incubations as unrecognisable forms to sheep GC. Also the involvement of the BMP15 pro-region protein is unclear. Indeed, the results from Chapter 7 indicate that rat BMP15 mRNA was detectable throughout the incubation period and the Western blotting studies showed that a pro-mature form of rat BMP15 protein was identified in oocyte lysates. Previous in vitro studies have shown that the pro-region protein of BMP15 can have an important role in GDF9BMP15 protein interaction (McIntosh, Lun et al. 2008). Current studies by Dr Joy McIntosh and colleagues are evaluating the effects of pro-mature regions of BMP15 on fertility in rodents using an immunization strategy (personal communication).

To further assess species' differences in functional activity of oocytes in vitro, QPCR methods were used to determine the relative expression levels of GDF9 and BMP15 mRNA in COC and in DO co-incubated with GC over a 24h period. GDF9 and BMP15 mRNA and protein have been shown in several studies to have similar expression profiles during follicular growth (Laitinen, Vuojolainen et al. 1998; Aaltonen, Laitinen et al. 1999; Eckery, Whale et al. 2002; McNatty, Reader et al. 2007). The evidence from both in situ hybridization and immuno-histochemical results shows that GDF9 and BMP15 mRNA and protein are produced exclusively within the ovary by oocytes (Laitinen, Vuojolainen et al. 1998; Jaatinen, Laitinen et 
al. 1999; Galloway, McNatty et al. 2000; Eckery, Whale et al. 2002). Thus, perhaps not surprisingly, GDF9 and BMP15 mRNA showed highly correlated levels of expression in both COC and in DO co-incubated with GC. The specificity of GDF9 and BMP15 mRNA with respect to its presence in oocytes and not $\mathrm{CC}$ was not tested in the current QPCR studies but has been confirmed independently in our laboratory in sheep, rat, mouse and the cow (Crawford, Heath et al. 2011).

In addition, Gdf9 mRNA levels were four times more abundant than Bmp15 mRNA levels in the rat, but showed equal abundance with BMP15 mRNA levels in sheep. Although the relative levels or abundance of mRNA is not directly indicative of presence of protein, the results from the Western blotting studies for BMP15 protein in Chapter 6 coupled with the evidence for lower rat Bmp15 mRNA levels further support the idea that mature rat BMP15 protein, if present, was indeed at very low amounts in the co-incubation studies, and a major reason for why rat oocytes did not stimulate ${ }^{3} \mathrm{H}$-thymidine incorporation by sheep GC.

\subsection{CC gene expression and incubation conditions}

In addition to GDF9 and BMP15 mRNA, QPCR was also used to measure probable markers of $\mathrm{CC}$ function in rat $\mathrm{COC}$ and sheep $\mathrm{COC}$ incubation. The specific marker genes were FSHR, KITL, CX43, CYCD2, LHR and AROM. LHR mRNA was undetectable in either species. AROM mRNA was undetectable in rat $\mathrm{COC}$ and declined over time in incubation in sheep COC. However, none of the other genes showed any significant changes in mRNA levels over time for either the rat or sheep COC incubation. 
These findings suggest that the oocytes may have acted as a stabilising influence on most of the CC genes investigated. However if this was the case, then it was of interest to note that this stabilising influence occurred despite a significant decline in GDF9 and BMP15 mRNA during the COC incubation period. It is known that throughout ovarian follicular growth, GDF9 and BMP15 mRNA are present in all oocytes of all follicles when assessed by in situ hybridization (McNatty, Lawrence et al. 2006). However, the relative expression levels at different developmental stages have not been quantified in most studies (McGrath, Esquela et al. 1995; Dube, Wang et al. 1998; Laitinen, Vuojolainen et al. 1998; Jaatinen, Laitinen et al. 1999; Bodensteiner, McNatty et al. 2000; Galloway, McNatty et al. 2000). In a study of sheep oocytes from primordial to antral follicles the relative expression levels of GDF9 and BMP15 mRNA increased until the antral stage (Feary, Juengel et al. 2007). In a more recent study of antral follicles, no change in relative expression levels of GDF9 or BMP15 mRNA was noted between small and large antral follicles (Crawford, Heath et al. 2011). Collectively, the published evidence suggests that the message levels do not decline in oocytes during follicular growth. In the present study, GDF9 and BMP15 mRNA in COC showed a tendency to decrease over time in incubation suggesting that the experimental model of 10 COC in $5 \mu 1$ M199 media was not a good approximate of what happens in vivo. In contrast, no significant changes in GDF9 or BMP15 mRNA levels over time in incubation were noted when 32 DO were

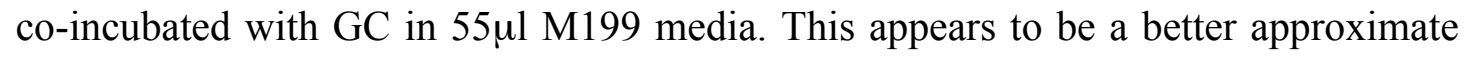
of what is likely to happen in vivo. This would also present a more indicative mRNA expression profile of the candidate genes during the co-incubations performed in the present study. It would be of interest to re-examine the mRNA expression profiles of both the oocyte-derived genes, BMP15 and GDF9, and the CC genes with $10 \mathrm{COC}$ in 
$15 \mu \mathrm{l}$ or $32 \mathrm{COC}$ in $55 \mu \mathrm{l}$ as the effect of oocyte concentration may be a critical variable. Also, a follow up study with DO oocytes from one species co-incubated with GC derived from another species may provide new insights into potential paracrine effects of GC on oocyte function.

\subsection{Conclusion}

In summary the findings from this study all point to significant differences between rats and sheep with respect to their regulation of BMP15 mRNA and protein in oocyte-cumulus cell communication. This conclusion can be made from the observations that: (1) co-incubations of rat oocytes with rat GC were the only combination not significantly affected by BMP15 protein neutralisation; (2) mature rat BMP15 protein was not detectable in rat oocyte lysates or spent media and; (3) rat Bmp15 mRNA levels were approximately four times less than rat Gdf9 mRNA levels. These findings add further compelling evidence to support a growing number of published studies suggesting that the $B M P 15$ gene is a major factor in regulating ovulation quota in mammals (Scaramuzzi, Adams et al. 1993; Bodensteiner, McNatty

et al. 2000; McNatty, Juengel et al. 2001; Hanrahan, Gregan et al. 2004; Juengel, Hudson et al. 2004; Hashimoto, Moore et al. 2005; McNatty, Smith et al. 2005; Shimasaki 2006; McNatty, Hudson et al. 2007).

In a recent study by McMahon, Hashimoto et al. (2008), litter size was found to remain unchanged when BMP15 protein was over-expressed in transgenic mice. Based on the 5:1 GDF9:BMP15 mRNA expression levels found in the current study, it is possible that if BMP15 is over-expressed, GDF9 would also be over-expressed as 
they appear to be tightly regulated (Table $7 \mathrm{~A}$ and Figures $7 \mathrm{G}$ and $\mathrm{H}$, see also Crawford, Heath et al. 2011).

However, McMahon, Hashimoto et al. (2008) did report that in the BMP15overexpressed transgenic mice there was a significant impact on early folliculogenesis, leading to an early onset of infertility. It is also possible that different levels of processing (e.g. unprocessed pro-mature) and/or glycosylation of the BMP15 protein may alter its activity (McIntosh, Lun et al. 2008; McMahon, Sharma et al. 2008) and thereby effect ovulation quota.

The potential involvement of the pro-region and pro-mature protein is likely to be another important level of regulation (McIntosh, Lun et al. 2008). Furthermore, GC of different species may have an inherent response to BMP15 protein that contributes to the determination of ovulation quota (Reader, Heath et al. 2011). Thus, BMP15 maybe an important contributor in the control of ovulation quota but more studies are required to fully understand this very complex process. 


\section{Appendix 1 MATERIALS}

\section{A1.1 Stock Solutions}

$10 \%(\mathrm{w} / \mathrm{v})$ Ammonium persulphate

$1 \mathrm{~g}$ of ammonium persulphate was dissolved in $10 \mathrm{ml}$ distilled water and stored at $4^{\circ} \mathrm{C}$.

Bicarbonate buffered-M199 (M199)

Pre-made, sodium bicarbonate buffered Medium 199 was purchased from SigmaAldrich (Cat. No. M4530). It was stored at $4^{\circ} \mathrm{C}$. On the day of use, $10 \mu \mathrm{M}$ Milrinone was added to prevent oocytes undergoing germinal vesicle breakdown (GVB) during the incubation interval following the suggestion by Sugiura, Pendola et al. (2005).

Bis-acrylamide mix

Bis-acrylamide mix was purchased from BioRad (Cat. No. 161-0158) and was stored at $4^{\circ} \mathrm{C}$.

$5 \%$ Blotto solution

$2.5 \mathrm{~g}$ of non-fat milk powder (purchased from the supermarket), was dissolved in $50 \mathrm{~mL}$ of low salt buffer (see below).

Diethylpyrocarbonate (DEPC) water

$1 \mathrm{ml}$ of DEPC was dissolved in 1L of distilled water and stirred overnight in the fume hood and sterilized under standard autoclave conditions. 
Dimethyl sulphoxide (DMSO)

DMSO was purchased from Sigma-Aldrich (Cat. No. D2650) and was stored at room temperature.

Ethylenediaminetetraacetic acid (0.5M: ETDA)

EDTA, 9.306g, (BDH, Cat. No. 100935V) was dissolved in DEPC water and adjusted to $\mathrm{pH} 8$ with $5 \mathrm{M}$ sodium hydroxide $(\mathrm{NaOH})$. This solution was made to a final volume of $50 \mathrm{ml}$ in DEPC water and was stored at room temperature.

$70 \%$ Ethanol

100\% analytical grade ethanol (Sigma-Aldrich, Cat. No. 24106, molecular weight: 46.07) was diluted to $70 \%$ in DEPC water and was stored at room temperature.

Electrical chemi-luminescence (ECL) reagents

Luminol (5-amino-2,3-dihydro-1,4-phthalazinedione) was purchased from SigmaAldrich (Cat. No. 45106182) and made to $250 \mathrm{mM}$ by dissolving $0.44 \mathrm{~g}$ in $10 \mathrm{ml}$ DMSO. It was stored at $-20^{\circ} \mathrm{C}$.

P-coumaric acid was purchased from Sigma-Aldrich (Cat. No. C9008-5G) and made to $90 \mathrm{mM}$ by dissolving $0.15 \mathrm{~g}$ in $10 \mathrm{ml}$ DMSO. It was stored at $-20^{\circ} \mathrm{C}$.

Two 10ml ECL mixtures were made and mixed just before use. The first consisted of $8.85 \mathrm{ml}$ distilled water, $1 \mathrm{ml} 1 \mathrm{M}$ Tris $\mathrm{pH} 8.5,100 \mu 1250 \mathrm{mM}$ luminol and $44 \mu 190 \mathrm{mM} \mathrm{p}$ coumaric acid. The second consisted of $9 \mathrm{ml}$ distilled water, $1 \mathrm{ml} 1 \mathrm{M}$ Tris $\mathrm{pH} 8.5$ and $6 \mu l$ hydrogen peroxide (Scharlau NZ, Cat. No. HI0136100). 
Isopropanol

RNAse-free isopropanol was purchased from AppliChem through the Global Science distributor (Cat. No. APPA39281000PE) and stored at room temperature.

Leibovitz media

Leibovitz media was purchased from Invitrogen (Cat. No. 11415049) and stored at $4^{\circ} \mathrm{C}$. Bovine serum albumin (BSA, ICPbio, Cat. No. ABRE-010) was added to a final concentration of $1 \mathrm{mg} / \mathrm{ml}$ and $0.044 \mathrm{mg} / \mathrm{ml}$ 3-Isobutyl-1-methyl-xanthine (MIX, see below) was added to prevent oocyte GVB during dissection. A final concentration of $100 \mathrm{U}$ penicillin and $100 \mu \mathrm{g}$ streptomycin per $\mathrm{ml}$ was also added to the media.

Low salt buffer

A $1 \mathrm{~L}$ low salt buffer was made by mixing $20 \mathrm{ml} 1 \mathrm{M}$ Tris with $9 \mathrm{~g}$ sodium chloride (NaCl, Thermo Fisher Scientific NZ, Cat. No. AC19409-0010) and 1ml Tween 20 (molecular weight: $1227.72 \mathrm{~g} / \mathrm{mol}$ ). This was stored at room temperature.

3-Isobutyl-1-methyl-xanthine (MIX)

MIX powder was purchased from Sigma-Aldrich (Cat. No. 15879) and was stored at $20^{\circ} \mathrm{C}$.

Milrinone

Milrinone powder was purchased from Sigma-Aldrich (Cat. No. M4659-50MG) and stored at $4^{\circ} \mathrm{C}$. A stock solution of $10 \mathrm{mM}$ was made by dissolving in the milrinone in DMSO. The stock solution was stored at $-20^{\circ} \mathrm{C}$. 
$5 \mathrm{M}$ Sodium hydroxide $(\mathrm{NaOH})$

The solution was prepared by dissolving $30 \mathrm{~g}$ of $\mathrm{NaOH}$ (molecular weight: $40 \mathrm{~g} / \mathrm{mol}$ ) in $150 \mathrm{ml}$ DEPC water. The solution was stored at room temperature.

Penicillin-Streptomycin

Pen-Strep was purchased from Invitrogen (Cat. No. 15140-122) and used at a final concentration of $100 \mathrm{U}$ penicillin and $100 \mu \mathrm{g}$ streptomycin per $\mathrm{ml}$.

Phosphate buffered saline (PBS)

Sterile PBS was made by dissolving a Sigma-Aldrich PBS tablet (Cat. No. P4417$100 \mathrm{TAB}$ ) in $200 \mathrm{~mL}$ distilled water and sterilized under standard autoclave conditions.

Ponceau S stain

Ponceau $\mathrm{S} \quad 1 \mathrm{~g}$

Acetic acid $2 \mathrm{ml}$

Ponceau S stain was purchased from BDH Laboratory Supplies (Cat. No. 440832H) made to $200 \mathrm{ml}$ with distilled water and $2 \mathrm{ml}$ acetic acid was added. It was stored at room temperature.

\section{TE Buffer}

The buffer was prepared by adding 0.1211g Tris (Invitrogen, Cat. No. 15504-020, molecular weight: $121.14 \mathrm{~g} / \mathrm{mol}$ ) to $200 \mu \mathrm{l}$ of $0.5 \mathrm{M}$ EDTA and adjusting the $\mathrm{pH}$ to 8 with $\mathrm{HCl}$. The solution was made up to a volume of $100 \mathrm{ml}$ with DEPC water so that 
the final concentration was $10 \mathrm{mM}$ Tris- $\mathrm{HCl}, 1 \mathrm{mM}$ EDTA. The buffer was stored at room temperature.

110-18-9 N, N, N',N'-tetramethylethylenediamine (TEMED)

TEMED was purchased from BioRad (Cat. No. 161-0800) and stored at $4^{\circ} \mathrm{C}$.

$1 \mathrm{M}$ Tris buffer

The $1 \mathrm{M}$ Tris buffer was made by dissolving $60.7 \mathrm{~g}$ Tris in distilled water and adjusted to $\mathrm{pH} 7.5$ with concentrated $\mathrm{HCl}$. The final volume of the solution was $500 \mathrm{ml}$. The source of Tris is the same as mentioned above.

10x Tris-glycine transfer buffer

Tris base $(3.03 \%) \quad 30.3 \mathrm{~g}$

Glycine $(11.4 \%) \quad 114 \mathrm{~g}$

A 10x stock Tris-glycine transfer buffer was made by dissolving Tris and Glycine (BDH, Cat. No. 101196X, molecular weight: $75.07 \mathrm{~g} / \mathrm{mol}$ ) in 1L distilled water and stored at room temperature. The source of Tris is the same as mentioned above.

For $2 \mathrm{~L}$ of $1 \mathrm{x}$ transfer buffer: $200 \mathrm{ml}$ of the $10 \mathrm{x}$ Tris-glycine stock was added to $400 \mathrm{ml}$ methanol and made to $2 \mathrm{~L}$ with distilled water. The transfer buffer is applied as a chilled solution before use and was stored at $4^{\circ} \mathrm{C}$.

Tris- $\mathrm{HCl}$

The source of Tris is the same as mentioned above. 
To make $1.5 \mathrm{M}$ Tris- $\mathrm{HCl}$ at $\mathrm{pH} 8.8,36.34 \mathrm{~g}$ of Tris was dissolved in $160 \mathrm{ml}$ distilled water and adjusted to $\mathrm{pH} 8.8$ with concentrated $\mathrm{HCl}$. It was made to a final volume of $200 \mathrm{ml}$ and stored at $4^{\circ} \mathrm{C}$.

To make $0.5 \mathrm{M}$ Tris- $\mathrm{HCl}$ at $\mathrm{pH} 6.8,6.06 \mathrm{~g}$ of Tris was dissolved in $80 \mathrm{ml}$ distilled water and adjusted to $\mathrm{pH} 6.8$ with concentrated $\mathrm{HCl}$. This buffer was then made up to $100 \mathrm{ml}$ and stored at $4^{\circ} \mathrm{C}$.

$10 \%(\mathrm{w} / \mathrm{v})$ Sodium Dodecyl Sulphate (SDS)

The solution was made by dissolving $20 \mathrm{~g}$ of SDS, purchased from BDH (Cat. No. $444464 \mathrm{~T}$ ), in $200 \mathrm{ml}$ distilled water and was stored at room temperature.

Strip (acid glycine) buffer

Glycine $(0.4 \mathrm{M}) \quad 1.5 \mathrm{~g}$

$\operatorname{SDS}(0.2 \% \mathrm{w} / \mathrm{v}) \quad 0.1 \mathrm{~g}$

Tween $20(2 \%) \quad 1 \mathrm{ml}$

The glycine and SDS were dissolved in $40 \mathrm{ml}$ distilled water and the $\mathrm{pH}$ was adjusted to 2.2 using concentrated $\mathrm{HCl}$ before the Tween 20 was added. This buffer was made to a final volume of $50 \mathrm{ml}$. It was made and used on the same day. The sources of Glycine, SDS and Tween 20 are the same as mentioned above.

Western blotting sample buffer

Distilled water $(10.81 \%) \quad 0.8 \mathrm{ml}$

0.5M Tris-HCl pH6.8 (13.51\%) $\quad 1 \mathrm{ml}$

Glycerol (10.81\%) $\quad 0.8 \mathrm{ml}$ 
$10 \% \operatorname{SDS}(62.16 \%) \quad 4.6 \mathrm{ml}$

$100 \mathrm{mM}$ DTT $(1.08 \% \mathrm{w} / \mathrm{v}) \quad 114 \mathrm{mg}$

$1 \% \operatorname{BPB}(2.70 \%) \quad 0.2 \mathrm{ml}$

Stock sample buffers were made to $5 \mathrm{x}$ concentrations and stored at $-20^{\circ} \mathrm{C}$. Dithiothreitol (DTT) was purchased from BDH (Cat. No. 443852A). Bromophenol blue sodium salt (BPB) was purchased from Sigma-Aldrich (Cat. No. BE131-5G) and prepared as a $1 \%(\mathrm{w} / \mathrm{v})$ BPB solution. Glycerol was purchased from Sigma-Aldrich (Cat. No. G5516-1L) and all of these were stored at room temperature. The source of SDS was the same as mentioned above.

5x SDS PAGE running buffer

Glycine $(7.2 \%) \quad 72 \mathrm{~g}$

Tris $(1.5 \%) \quad 15 \mathrm{~g}$

$\operatorname{SDS}(0.5 \%) \quad 5 \mathrm{~g}$

The running buffer was made in distilled water and the $\mathrm{pH}$ was adjusted to 8.3 with concentrated $\mathrm{HCl}$ and then made to a final volume of $1 \mathrm{~L}$ and stored at room temperature. It was diluted before use by diluting $100 \mathrm{ml} 5 \mathrm{x}$ stock running buffer in $400 \mathrm{ml}$ distilled water. The sources of Glycine, Tris and SDS were the same as mentioned above. 


\section{REFERENCES}

Aaltonen, J., M. P. Laitinen, et al. (1999). "Human growth differentiation factor 9 (GDF-9) and its novel homolog GDF-9B are expressed in oocytes during early folliculogenesis." J Clin Endocrinol Metab 84(8): 2744-2750.

Ackert, C. L., J. E. I. Gittens, et al. (2001). "Intercellular communication via connexin43 gap junctions is required for ovarian folliculogenesis in the mouse." Developmental Biology 233(2): 258-270.

Adashi, E. Y. and A. J. Hsueh (1982). "Estrogens augment the stimulation of ovarian aromatase activity by follicle-stimulating hormone in cultured rat granulosa cells." Journal of Biological Chemistry 257(11): 6077-6083.

Bäckström, C. T., A. S. McNeilly, et al. (1982). "Pulsatile secretion of LH, FSH, prolactin, oestradiol and progesterone during the human menstrual cycle." Clinical Endocrinology 17(1): 29-42.

Baird, D. T., I. A. Swanston, et al. (1981). "Relationship between LH, FSH, and prolactin concentration and the secretion of androgens and estrogens by the preovulatory follicle in the ewe." Biology of Reproduction 24(5): 1013-1025.

Baker, T. G. (1963). "Aquantitative and cytological study of germ cells in human ovaries." Proceedings of the Royal Society of London. Series B, Biological Sciences 158(972): 417-433.

Bebbere, D., L. Bogliolo, et al. (2008). "Expression pattern of zygote arrest 1 (ZAR1), maternal antigen that embryo requires (MATER), growth differentiation factor 9 (GDF9) and bone morphogenetic protein 15 (BMP15) genes in ovine oocytes and in vitro-produced preimplantation embryos." Reprod. Fertil. Dev. 20(8): 908-915. 
Bilezikjian, L. M., A. L. Blount, et al. (2004). "Autocrine/paracrine regulation of pituitary function by activin, inhibin and follistatin." Molecular and Cellular Endocrinology 225(1-2): 29-36.

Bilezikjian, L. M., A. L. Blount, et al. (2006). "Pituitary actions of ligands of the TGF- $\{$ beta $\}$ family: activins and inhibins." Reproduction 132(2): 207-215.

Bodensteiner, K. J., C. M. Clay, et al. (1999). "Molecular cloning of the ovine growth differentiation factor-9 gene and expression of growth differentiation factor-9 in ovine and bovine ovaries." Biol Reprod 60(2): 381-386.

Bodensteiner, K. J., K. P. McNatty, et al. (2000). "Expression of growth and differentiation factor-9 in the ovaries of fetal sheep homozygous or heterozygous for the Inverdale prolificacy gene (FecXI)." Biol Reprod 62(6): $1479-1485$.

Bodin, L., E. Di Pasquale, et al. (2007). "A novel mutation in the bone morphogenetic protein 15 gene causing defective srotein Secretion is associated with both increased ovulation rate and sterility in Lacaune sheep." Endocrinology 148(1): 393-400.

Braw-Tal, R., K. P. McNatty, et al. (1993). "Ovaries of ewes homozygous for the Xlinked Inverdale gene (FecXI) are devoid of secondary and tertiary follicles but contain many abnormal structures." Biol Reprod 49(5): 895-907.

Campbell, B. and D. Baird (2001). "Inhibin A is a follicle stimulating hormoneresponsive marker of granulosa cell differentiation, which has both autocrine and paracrine actions in sheep." J Endocrinol 169(2): 333-345.

Carabatsos, M. J., J. Elvin, et al. (1998). "Characterization of oocyte and follicle development in growth differentiation factor-9-deficient mice." Developmental Biology 204(2): 373-384. 
Chang, H., C. W. Brown, et al. (2002). "Genetic analysis of the mammalian transforming growth factor- $\beta$ superfamily." Endocr Rev 23(6): 787-823.

Chiles, T. C. (2004). "Regulation and function of cyclin D2 in B lymphocyte subsets." The Journal of Immunology 173(5): 2901-2907.

Conti, M., C. B. Andersen, et al. (1998). "Role of cyclic nucleotide phosphodiesterases in resumption of meiosis." Molecular and Cellular Endocrinology 145(1-2): 9-14.

Crawford, J. L., D. A. Heath, et al. (2011). "Oocytes in sheep homozygous for a mutation in bone morphogenetic protein receptor $1 \mathrm{~B}$ express lower mRNA levels of bone morphogenetic protein 15 but not growth differrentiation factor 9." Reproduction epub ahead of print.

Davis, G. H., J. C. McEwan, et al. (1991). "Evidence for the presence of a major gene influencing ovulation rate on the $\mathrm{X}$ chromosome of sheep." Biology of Reproduction 44(4): 620-624.

Davis, G. H., J. C. McEwan, et al. (1992). "Infertility due to bilateral ovarian hypoplasia in sheep homozygous (FecXI FecXI) for the Inverdale prolificacy gene located on the X chromosome." Biology of Reproduction 46(4): 636-640.

de Caestecker, M. (2004). "The transforming growth factor-[beta] superfamily of receptors." Cytokine \& Growth Factor Reviews 15(1): 1-11.

Di Pasquale, E., P. Beck-Peccoz, et al. (2004). "Hypergonadotropic ovarian failure associated with an inherited mutation of human bone morphogenetic protein15 (BMP15) gene." Am J Hum Genet 75(1): 106-111.

Diaz, F. J., K. Wigglesworth, et al. (2007). "Oocytes are required for the preantral granulosa cell to cumulus cell transition in mice." Developmental Biology 305(1): 300-311. 
Dijke, P. t. and C. S. Hill (2004). "New insights into TGF-[beta]-Smad signalling." Trends in Biochemical Sciences 29(5): 265-273.

Dixit, H., L. Rao, et al. (2005). "Mutational screening of the coding region of growth differentiation factor 9 gene in Indian women with ovarian failure." Menopause 12(6): 749-754.

Dixit, H., L. Rao, et al. (2006). "Missense mutations in the BMP15 gene are associated with ovarian failure." Human Genetics 119(4): 408-415.

Dong, J., D. F. Albertini, et al. (1996). "Growth differentiation factor-9 is required during early ovarian folliculogenesis." Nature 383(6600): 531-535.

Drummond, A. E., M. T. Le, et al. (2002). "Expression and localization of activin receptors, Smads, and Bglycan to the postnatal rat ovary." Endocrinology 143(4): 1423-1433.

Drummond, A. (2005). "TGF $\beta$ signalling in the development of ovarian function." Cell and Tissue Research 322(1): 107-115.

Dube, J. L., P. Wang, et al. (1998). "The bone morphogenetic protein 15 gene is Xlinked and expressed in oocytes." Molecular Endocrinology 12(12): 18091817.

Duffy, D. M. (2003). "Growth differentiation factor-9 is expressed by the primate follicle throughout the periovulatory interval." Biology of Reproduction 69(2): 725-732.

Dunkel, L., J. L. Tilly, et al. (1994). "Follicle-stimulating hormone receptor expression in the rat ovary: increases during prepubertal development and regulation by the opposing actions of transforming growth factors beta and alpha." Biology of Reproduction 50(4): 940-948. 
Durlinger, A. L. L., P. Kramer, et al. (1999). "Control of primordial follicle recruitment by anti-mullerian hormone in the mouse ovary." Endocrinology 140(12): 5789-5796.

Durlinger, A. L. L., M. J. G. Gruijters, et al. (2001). "Anti-mullerian hormone attenuates the effects of FSH on follicle development in the mouse ovary." Endocrinology 142(11): 4891-4899.

Eckery, D. C., L. J. Whale, et al. (2002). "Expression of mRNA encoding growth differentiation factor 9 and bone morphogenetic protein 15 during follicular formation and growth in a marsupial, the brushtail possum (Trichosurus vulpecula)." Molecular and Cellular Endocrinology 192(1-2): 115-126.

Edwards, S. J., K. L. Reader, et al. (2008). "The cooperative effect of growth and differentiation factor-9 and bone morphogenetic protein (BMP)-15 on granulosa cell function is modulated primarily through BMP receptor II." Endocrinology 149(3): 1026-1030.

Elvin, J. A., A. T. Clark, et al. (1999). "Paracrine actions of growth differentiation factor-9 in the mammalian ovary." Mol Endocrinol 13(6): 1035-1048.

Elvin, J. A., C. Yan, et al. (1999). "Molecular characterization of the follicle defects in the growth differentiation factor 9-deficient ovary." Mol Endocrinol 13(6): $1018-1034$.

Eppig, J. J., K. Wigglesworth, et al. (1997). "Murine oocytes suppress expression of luteinizing hormone receptor messenger ribonucleic acid by granulosa cells." Biol Reprod 56(4): 976-984.

Eppig, J. J. (2001). "Oocyte control of ovarian follicular development and function in mammals." Reproduction 122(6): 829-838. 
Eppig, J. J., K. Wigglesworth, et al. (2002). "The mammalian oocyte orchestrates the rate of ovarian follicular development." PNAS 99(5): 2890-2894.

Eppig, J. J., F. L. Pendola, et al. (2005). "Mouse oocytes regulate metabolic cooperativity between granulosa cells and oocytes: amino acid transport." Biology of Reproduction 73(2): 351-357.

Erickson, G. F. and S. Shimasaki (2000). "The role of the oocyte in folliculogenesis." Trends in Endocrinology and Metabolism 11(5): 193-198.

Feary, E. S., J. L. Juengel, et al. (2007). "Patterns of expression of messenger RNAs encoding GDF9, BMP15, TGFBR1, BMPR1B, and BMPR2 during follicular development and characterization of ovarian follicular populations in ewes carrying the Woodlands FecX2W mutation." Biology of Reproduction: biolreprod.107.062752.

Findlay, J. K. (1993). "An update on the roles of inhibin, activin, and follistatin as local regulators of folliculogenesis." Biology of Reproduction 48(1): 15-23.

Fitzpatrick, S. L., D. M. Sindoni, et al. (1998). "Expression of growth differentiation factor-9 messenger ribonucleic acid in ovarian and nonovarian rodent and human tissues." Endocrinology 139(5): 2571-2578.

Fraser, H. M., N. P. Groome, et al. (1999). "Follicle-stimulating hormone-inhibin B interactions during the follicular phase of the primate menstrual cycle revealed by gonadotropin-releasing hormone antagonist and antiestrogen treatment." Journal of Clinical Endocrinology Metabolism 84(4): 1365-1369.

Fukuda, M., K. Miyamoto, et al. (1986). "Isolation of bovine follicular fluid inhibin of about 32 kDa." Molecular and Cellular Endocrinology 44(1): 55-60. 
Fuller, P. J. and S. Chu (2004). "Signalling pathways in the molecular pathogenesis of ovarian granulosa cell tumours." Trends in Endocrinology and Metabolism 15(3): 122-128.

Galloway, S. M., K. P. McNatty, et al. (2000). "Mutations in an oocyte-derived growth factor gene (BMP15) cause increased ovulation rate and infertility in a dosage-sensitive manner." Nat Genet 25(3): 279-283.

Gilchrist, R. B., L. J. Ritter, et al. (2001). "Mouse oocyte mitogenic activity is developmentally coordinated throughout folliculogenesis and meiotic maturation." Developmental Biology 240(1): 289-298.

Gilchrist, R. B., M. P. Morrissey, et al. (2003). "Comparison of oocyte factors and transforming growth factor-[beta] in the regulation of DNA synthesis in bovine granulosa cells." Molecular and Cellular Endocrinology 201(1-2): 8795.

Gilchrist, R. B., L. J. Ritter, et al. (2004). "Immunoneutralization of growth differentiation factor 9 reveals it partially accounts for mouse oocyte mitogenic activity." Biology of Reproduction 71(3): 732-739.

Gilchrist, R. B., L. J. Ritter, et al. (2004). "Oocyte-somatic cell interactions during follicle development in mammals." Animal Reproduction Science 82-83: 431446.

Gilchrist, R. B., L. J. Ritter, et al. (2006). "Molecular basis of oocyte-paracrine signalling that promotes granulosa cell proliferation." Journal of Cell Science 119(18): 3811-3821.

Gilchrist, R. B. and J. G. Thompson (2007). "Oocyte maturation: emerging concepts and technologies to improve developmental potential in vitro." Theriogenology 67(1): 6-15. 
Gilchrist, R. B., M. Lane, et al. (2008). "Oocyte-secreted factors: regulators of cumulus cell function and oocyte quality." Hum Reprod Update 14(2): 159177.

Glister, C., N. P. Groome, et al. (2006). "Bovine follicle development is associated with divergent changes in activin-A, inhibin-A and follistatin and the relative abundance of different follistatin isoforms in follicular fluid." $\mathrm{J}$ Endocrinol 188(2): $215-225$.

Gruijters, M. J. G., J. A. Visser, et al. (2003). "Anti-Mullerian hormone and its role in ovarian function." Molecular and Cellular Endocrinology 211(1-2): 85-90.

Gueripel, X., V. Brun, et al. (2006). "Oocyte bone morphogenetic protein 15, but not growth differentiation factor 9 , is increased during gonadotropin-induced follicular development in the immature mouse and is associated with cumulus oophorus expansion." Biology of Reproduction 75(6): 836-843.

Gui, L.-M. and I. M. Joyce (2005). "RNA interference evidence that growth differentiation factor-9 mediates oocyte regulation of cumulus expansion in mice." Biology of Reproduction 72(1): 195-199.

Hanrahan, J. P., S. M. Gregan, et al. (2004). "Mutations in the genes for oocytederived growth factors GDF9 and BMP15 are associated with both increased ovulation rate and sterility in Cambridge and Belclare sheep (Ovis aries)." Biol Reprod 70(4): 900-909.

Harrison, C. A., P. C. Gray, et al. (2005). "Antagonists of activin signaling: mechanisms and potential biological applications." Trends in Endocrinology and Metabolism 16(2): 73-78.

Hashimoto, O., R. K. Moore, et al. (2005). "Posttranslational procession of mouse and human BMP-15: potential implication in the determination of ovulation 
quota." Proceedings of the National Academy of Sciences 102(15): 54265431.

Hashimoto, H., S. Uji, et al. (2007). "Flounder and fugu have a single lefty gene that covers the functions of lefty 1 and lefty 2 of zebrafish during L-R patterning." Gene 387(1-2): 126-132.

Hillier, S. G. and F. Miró (1993). "Inhibin, activin, and follistatin potential roles in ovarian physiologya." Annals of the New York Academy of Sciences 687(1): 29-38.

Hotten, G., H. Neidhardt, et al. (1995). "Cloning of a new member of the TGF-[beta] family: a putative new activin $\beta \mathrm{C}$ chain." Biochemical and Biophysical Research Communications 206(2): 608-613.

Hsueh, A. J., K. D. Dahl, et al. (1987). "Heterodimers and homodimers of inhibin subunits have different paracrine action in the modulation of luteinizing hormone-stimulated androgen biosynthesis." PNAS 84(14): 5082-5086.

Huang, Q., A. P. Cheung, et al. (2009). "Effects of growth differentiation factor 9 on cell cycle regulators and ERK42/44 in human granulosa cell proliferation." Am J Physiol Endocrinol Metab 296(6): E1344-1353.

Hussein, T. S., J. G. Thompson, et al. (2006). "Oocyte-secreted factors enhance oocyte developmental competence." Developmental Biology 296(2): 514-521.

Hutchinson, L. A., J. K. Findlay, et al. (1987). "Effects of bovine inhibin, transforming growth factor-[beta] and bovine activin-A on granulosa cell differentiation." Biochemical and Biophysical Research Communications 146(3): 1405-1412.

Jaatinen, R., M. P. Laitinen, et al. (1999). "Localization of growth differentiation factor-9 (GDF-9) mRNA and protein in rat ovaries and cDNA cloning of rat 
GDF-9 and its novel homolog GDF-9B." Molecular and Cellular Endocrinology 156(1-2): 189-193.

Joyce, I. M., F. L. Pendola, et al. (1999). "Oocyte regulation of Kit ligand expression in mouse ovarian follicles." Developmental Biology 214(2): 342-353.

Joyce, I. M., A. T. Clark, et al. (2000). "Comparison of recombinant growth differentiation factor-9 and oocyte regulation of KIT ligand messenger ribonucleic acid expression in mouse ovarian follicles." Biol Reprod 63(6): $1669-1675$.

Juengel, J. L., L. D. Quirke, et al. (2000). "Gene expression in abnormal ovarian structures of ewes homozygous for the inverdale prolificacy gene." Biology of Reproduction 62(6): 1467-1478.

Juengel, J. L., H. R. Sawyer, et al. (2002). "Origins of follicular cells and ontogeny of steroidogenesis in ovine fetal ovaries." Molecular and Cellular Endocrinology 191(1): $1-10$.

Juengel, J. L., N. L. Hudson, et al. (2002). "Growth differentiation factor 9 and bone morphogenetic protein 15 are essential for ovarian follicular development in sheep." Biol Reprod 67(6): 1777-1789.

Juengel, J. L., A. Bibby, et al. (2004). "The role of transforming growth factor-beta (TGF- $\beta$ ) during ovarian follicular development in sheep." Reproductive Biology and Endocrinology 2(1): 78.

Juengel, J. L., K. J. Bodensteiner, et al. (2004). "Physiology of GDF9 and BMP15 signalling molecules." Animal Reproduction Science 82-83: 447-460.

Juengel, J. L., N. L. Hudson, et al. (2004). "Effects of immunization against bone morphogenetic protein 15 and growth differentiation factor 9 on ovulation 
rate, fertilization, and pregnancy in ewes." Biology of Reproduction 70(3): $557-561$.

Juengel, J. L. and K. P. McNatty (2005). "The role of proteins of the transforming growth factor- $\beta$ superfamily in the intraovarian regulation of follicular development." Human Reproduction Update 11(2): 144-161.

Kaivo-Oja, N., J. Bondestam, et al. (2003). "Growth differentiation factor-9 induces Smad2 activation and inhibin B production in cultured human granulosa-luteal cells." Journal of Clinical Endocrinology Metabolism 88(2): 755-762.

Keutmann, H. T., A. L. Schneyer, et al. (2004). "The role of follistatin domains in

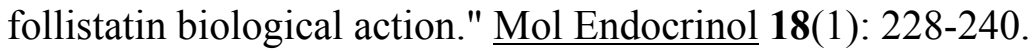

Kingsley, D. M. (1994). "The TGF-beta superfamily: new members, new receptors, and new genetic tests of function in different organisms." Genes and Development 8(2): 133-146.

Knight, P. G. (1996). "Roles of inhibins, activins, and follistatin in the female reproductive system." Frontiers in Neuroendocrinology 17(4): 476-509.

Knight, P. and C. Glister (2001). "Potential local regulatory functions of inhibins, activins and follistatin in the ovary." Reproduction 121(4): 503-512.

Knight, P. G. and C. Glister (2003). "Local roles of TGF-[beta] superfamily members in the control of ovarian follicle development." Animal Reproduction Science 78(3-4): 165-183.

Knight, P. G. and C. Glister (2006). "TGF- $\beta$ superfamily members and ovarian follicle development." Reproduction 132(2): 191-206.

Laissue, P., S. Christin-Maitre, et al. (2006). "Mutations and sequence variants in GDF9 and BMP15 in patients with premature ovarian failure." Eur J Endocrinol 154(5): 739-744. 
Laitinen, M., K. Vuojolainen, et al. (1998). "A novel growth differentiation factor-9 (GDF9) related factor is co-expressed with GDF9 in mouse oocytes during folliculogenesis." Mech Dev. 78: 135-140.

Lane, A. H. and P. K. Donahoe (1998). "New insights into mullerian inhibiting substance and its mechanism of action." Journal of Endocrinology 158(1): 1-6.

Lee, W.-S., S.-J. Yoon, et al. (2004). "Effects of bone morphogenetic protein-7 (BMP-7) on primordial follicular growth in the mouse ovary." Molecular Reproduction and Development 69(2): 159-163.

Li, H.-K., T.-Y. Kuo, et al. (2008). "Differential gene expression of bone morphogenetic protein 15 and growth differentiation factor 9 during in vitro maturation of porcine oocytes and early embryos." Animal Reproduction Science 103(3-4): 312-322.

Li, R., R. J. Norman, et al. (2000). "Oocyte-secreted factor(s) determine functional differences between bovine mural granulosa cells and cumulus cells." Biology of Reproduction 63(3): 839-845.

Liao, W. X., R. K. Moore, et al. (2003). "Effect of intracellular interactions on the processing and secretion of bone morphogenetic protein-15 (BMP-15) and growth and differentiation factor-9. implication of the aberrant ovarian phenotype of BMP-15 mutant sheep." Journal of Biological Chemistry 278(6): $3713-3719$.

Liao, W. X., R. K. Moore, et al. (2004). "Functional and molecular characterization of naturally occurring mutations in the oocyte-secreted factors bone morphogenetic protein-15 and growth and differentiation factor-9." Journal of Biological Chemistry 279(17): 17391-17396. 
Ling, N., S.-Y. Ying, et al. (1986). "A homodimer of the [beta]-subunits of inhibin a stimulates the secretion of pituitary follicle stimulating hormone." Biochemical and Biophysical Research Communications 138(3): 1129-1137.

Liu, J., T. Kuulasmaa, et al. (2003). "Expression of betaglycan, an inhibin coreceptor, in normal human ovaries and ovarian sex cord-stromal tumors and its regulation in cultured human granulosa-luteal cells." J Clin Endocrinol Metab 88(10): 5002-5008.

Livak, K. J. and T. D. Schmittgen (2001). "Analysis of relative gene expression data using real-time quantitative PCR and the 2- $\Delta \Delta \mathrm{CT}$ method." Methods 25(4): 402-408.

Logan, K. A., J. L. Juengel, et al. (2002). "Onset of steroidogenic enzyme gene expression during ovarian follicular development in sheep." Biology of Reproduction 66(4): 906-916.

Lundy, T., P. Smith, et al. (1999). "Populations of granulosa cells in small follicles of the sheep ovary." J. Reprod. Fert. 115: 215-262.

MacConell, L. A., A. M. O. Leal, et al. (2002). "The distribution of betaglycan protein and mRNA in rat brain, pituitary, and gonads: implications for a role for betaglycan in inhibin-mediated reproductive functions." Endocrinology 143(3): 1066-1075.

Marsters, P., N. R. Kendall, et al. (2003). "Temporal relationships between FSH receptor, type 1 insulin-like growth factor receptor, and aromatase expression during FSH-induced differentiation of bovine granulosa cells maintained in serum-free culture." Molecular and Cellular Endocrinology 203(1-2): 117-127. 
Martinez-Royo, A., E. Dervishi, et al. (2009). "Freemartinism and FecXR allele determination in replacement ewes of the Rasa Aragonesa sheep breed by duplex PCR." Theriogenology 72(8): 1148-1152.

Mason, A. J., H. D. Niall, et al. (1986). "Structure of two human ovarian inhibins." Biochemical and Biophysical Research Communications 135(3): 957-964.

Matzuk, M. M., M. J. Finegold, et al. (1992). "[alpha]-lnhibin is a tumour-suppressor gene with gonadal specificity in mice." Nature 360(6402): 313-319.

Matzuk, M. M., T. R. Kumar, et al. (1995). "Different phenotypes for mice deficient in either activins or activin receptor type II." Nature 374(6520): 356-360.

Matzuk, M. M., K. H. Burns, et al. (2002). "Intercellular communication in the mammalian ovary: oocytes carry the conversation." Science 296(5576): 21782180.

Mazerbourg, S., C. Klein, et al. (2004). "Growth differentiation factor-9 signaling is mediated by the type I receptor, activin receptor-like kinase 5." $\underline{\text { Mol }}$ Endocrinol 18(3): 653-665.

Mazerbourg, S. and A. J. W. Hsueh (2006). "Genomic analyses facilitate identification of receptors and signalling pathways for growth differentiation factor 9 and related orphan bone morphogenetic protein/growth differentiation factor ligands." Human Reproduction Update 12(4): 373-383.

McGee, E. A. and A. J. W. Hsueh (2000). "Initial and cyclic recruitment of ovarian follicles." Endocr Rev 21(2): 200-214.

McGrath, S. A., A. F. Esquela, et al. (1995). "Oocyte-specific expression of growth/differentiation factor-9." Mol Endocrinol 9(1): 131-136. 
McIntosh, C. J., S. Lun, et al. (2008). "The proregion of mouse BMP15 regulates the cooperative interactions of BMP15 and GDF9." Biology of Reproduction 79(5): 889-896.

McMahon, H. E., O. Hashimoto, et al. (2008). "Oocyte-specific over-expression of mouse BMP-15 leads to accelerated folliculogenesis and an early onset of acyclicity in transgenic mice." Endocrinology: en.2007-1550.

McMahon, H. E., S. Sharma, et al. (2008). "Phosphorylation of bone morphogenetic protein-15 and growth and differentiation factor-9 Plays a critical role in determining agonistic or antagonistic functions." Endocrinology 149(2): 812817.

McNatty, K. P. (1981). Ovarian follicular development from the onset of luteal regression in humans and sheep. Follicular Maturation and Ovulation. R. Rolland, E. V. van Hall, S. G. Hillier, K. P. McNatty and J. Schoemaker. Amsterdam, Excerpta Medica: 1-18.

McNatty, K. P., L. D. Quirke, et al. (2000). Ovarian development: fetus to puberty. Hormones and Women's Health: The Reproductive Years. L. A. Salamonsen. Australia, Harwood Academic Publishers. 1: 9-22.

McNatty, K. P., J. L. Juengel, et al. (2001). "Genetic mutations influencing ovulation rate in sheep." Reproduction, Fertility and Development 13(8): 549-555.

McNatty, K. P., J. L. Juengel, et al. (2005a). "Bone morphogenetic protein 15 and growth differentiation factor 9 co-operate to regulate granulosa cell function." Reproduction 129(4): 473-480.

McNatty, K. P., J. L. Juengel, et al. (2005b). "Bone morphogenetic protein 15 and growth differentiation factor 9 co-operate to regulate granulosa cell function in ruminants." Reproduction 129(4): 481-487. 
McNatty, K. P., P. Smith, et al. (2005). "Oocyte-expressed genes affecting ovulation rate." Molecular and Cellular Endocrinology 234(1-2): 57-66.

McNatty, K. P., S. Lawrence, et al. (2006). "Meat and Livestock Association Plenary Lecture 2005. Oocyte signalling molecules and their effects on reproduction in ruminants." Reproduction, Fertility and Development 18(4): 403-412.

McNatty, K. P., K. Reader, et al. (2007). Control of ovarian follicular development to the gonadotrophin-dependent phase: a 2006 perspective. Reproduction in Domestic Ruminants VI. J. L. Juengel, J. F. Murray and M. F. Smith. UK, Nottingham University Press. 64: 55-68.

McNatty, K. P., N. L. Hudson, et al. (2007). "The effects of immunizing sheep with different BMP15 or GDF9 peptide sequences on ovarian follicular activity and ovulation rate." Biology of Reproduction 76(4): 552-560.

McPherron, A. C. and S. J. Lee (1993). "GDF-3 and GDF-9: two new members of the transforming growth factor-beta superfamily containing a novel pattern of cysteines." Journal of Biological Chemistry 268(5): 3444-3449.

Messinis, I. E., C. I. Messini, et al. (2010). "The role of gonadotropins in the follicular phase." Annals of the New York Academy of Sciences 1205(1): 5-11.

Miro, F. and S. Hillier (1996). "Modulation of granulosa cell deoxyribonucleic acid synthesis and differentiation by activin." Endocrinology 137(2): 464-468.

Miyamoto, K., Y. Hasegawa, et al. (1985). "Isolation of porcine follicular fluid inhibin of $32 \mathrm{~K}$ daltons." Biochemical and Biophysical Research Communications 129(2): 396-403.

Miyoshi, T., F. Otsuka, et al. (2010). "Functional relationship between fibroblast growth factor- 8 and bone morphogenetic proteins in regulating steroidogenesis 
by rat granulosa cells." Molecular and Cellular Endocrinology 325(1-2): 8492.

Monteagudo, L. V., R. Ponz, et al. (2009). "A 17bp deletion in the Bone Morphogenetic Protein 15 (BMP15) gene is associated to increased prolificacy in the Rasa Aragonesa sheep breed." Animal Reproduction Science 110(1-2): 139-146.

Moore, R. K., F. Otsuka, et al. (2003). "Molecular Basis of Bone Morphogenetic Protein-15 Signaling in Granulosa Cells." Journal of Biological Chemistry 278(1): 304-310.

Mottershead, D. G., M. M. Pulkki, et al. (2008). "Characterization of recombinant human growth differentiation factor-9 signaling in ovarian granulosa cells." Molecular and Cellular Endocrinology 283(1-2): 58-67.

Nakamura, M., T. Minegishi, et al. (1993). "Effect of an activin A on folliclestimulating hormone $(\mathrm{FSH})$ receptor messenger ribonucleic acid levels and FSH receptor expressions in cultured rat granulosa cells." Endocrinology 133(2): 538-544.

Nakano, R., T. Mizuno, et al. (1975). "Growth of ovarian follicles in rats in the absence of gonadotrophins." J Reprod Fertil 45(3): 545-546.

Nicol, L., S. C. Bishop, et al. (2009). "Homozygosity for a single base-pair mutation in the oocyte-specific GDF9 gene results in sterility in Thoka sheep." Reproduction 138(6): 921-933.

Oda, S., S. Nishimatsu, et al. (1995). "Molecular cloning and functional analysis of a new activin beta subunit: a dorsal mesoderm-inducing activity in Xenopus." Biochemical and Biophysical Research Communications 210(2): 581-588. 
Otsuka, F., Z. Yao, et al. (2000). "Bone morphogenetic protein-15. Identification of target cells and biological functions." Journal of Biological Chemistry 275(50): 39523-39528.

Otsuka, F., S. Yamamoto, et al. (2001). "Bone morphogenetic protein-15 inhibits follicle-stimulating hormone (FSH) action by suppressing $\mathrm{FSH}$ receptor expression." Journal of Biological Chemistry 276(14): 11387-11392.

Otsuka, F. and S. Shimasaki (2002). "A negative feedback system between oocyte bone morphogenetic protein 15 and granulosa cell kit ligand: Its role in regulating granulosa cell mitosis." PNAS 99(12): 8060-8065.

Otsuka, F., K. J. McTavish, et al. (2011). "Integral role of GDF-9 and BMP-15 in ovarian function." Molecular Reproduction and Development 78(1): 9-21.

Oulad-Abdelghani, M., C. Chazaud, et al. (1998). "Stra3/lefty, a retinoic acidinducible novel member of the transforming growth factor-beta superfamily." Int. J. Dev. Biol. 42(1): 23-32.

Palmer, J. S., Z. Z. Zhao, et al. (2006). "Novel variants in growth differentiation mactor 9 in Mothers of dizygotic twins." J Clin Endocrinol Metab 91(11): 4713-4716.

Peng, X.-R., A. J. W. Hsueh, et al. (1991). "Localization of luteinizing hormone receptor messenger ribonucleic acid expression in ovarian cell types during follicle development and ovulation." Endocrinology 129(6): 3200-3207.

Peters, H., A. G. Byskov, et al. (1975). "Follicular growth: the basic event in the mouse and human ovary." J. Reprod. Fert. 45: 559-566.

Peters, H. and K. P. McNatty (1980). The Ovary: a correlation of struction and function in mammals. Berkeley and Los Angeles, University of California Press. 
Picton, H. M., B. K. Campbell, et al. (1999). "Maintenance of oestradiol production and expression of cytochrome P450 aromatase enzyme mRNA in long-term serum-free cultures of pig granulosa cells." J Reprod Fertil 115(1): 67-77.

Pierce, J. G. and T. F. Parsons (1981). "Glycoprotein hormones: structure and function." Annual Review of Biochemistry 50(1): 465-495.

Reader, K., D. A. Heath, et al. (2011). "Signalling pathways involved in the cooperative effects of ovine and murine GDF9 \& BMP15 stimulated

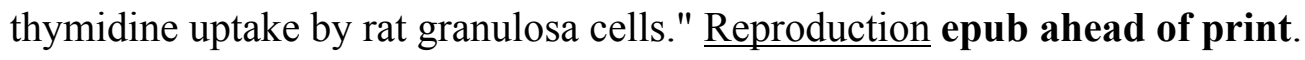

Rey, R., C. Lukas-Croisier, et al. (2003). "AMH/MIS: what we know already about the gene, the protein and its regulation." Molecular and Cellular Endocrinology 211(1-2): 21-31.

Reynolds, S. R. M. (1973). Blood and lymph vascular systems of the ovary. Handbook of Physiology: a critical, comprehensive presentation of physiological knowledge and concepts. R. O. Greep. Washington D. C., American Physiological Society. II: 261-316.

Robertson, D. M., L. M. Foulds, et al. (1985). "Isolation of inhibin from bovine follicular fluid." Biochemical and Biophysical Research Communications 126(1): $220-226$.

Robertson, D. M., F. L. de Vos, et al. (1986). "Isolation of a $31 \mathrm{kDa}$ form of inhibin from bovine follicular fluid." Molecular and Cellular Endocrinology 44(3): 271-277.

Rodgers, R. J. and H. F. Irving-Rodgers (2010). "Formation of the ovarian follicular antrum and follicular fluid." Biology of Reproduction 82(6): 1021-1029. 
Russell, D. L. and R. L. Robker (2007). "Molecular mechanisms of ovulation: coordination through the cumulus complex." Human Reproduction Update 13(3): 289-312.

Sánchez, F., T. Adriaenssens, et al. (2009). "Quantification of oocyte-specific transcripts in follicle-enclosed oocytes during antral development and maturation in vitro." Molecular Human Reproduction 15(9): 539-550.

Sawyer, H. R., P. Smith, et al. (2002). "Formation of ovarian follicles during fetal development in sheep." Biol Reprod 66(4): 1134-1150.

Scaramuzzi, R. J., N. R. Adams, et al. (1993). "A model for follicle selection and the determination of ovulation rate in the ewe." Reprod. Fertil. Dev. 5(5): 459478.

Scaramuzzi, R. J., D. T. Baird, et al. (2011). "Regulation of folliculogenesis and the determination of ovulation rate in ruminants." Reprod. Fertil. Dev. 23: 444467.

Schneider, J. E. (2004). "Energy balance and reproduction." Physiology \& Behavior 81(2): 289-317.

Schwall, R. H., A. J. Mason, et al. (1990). "Localization of inhibin/activin subunit mRNAs within the primate ovary." Mol Endocrinol 4(1): 75-79.

Senbon, S., Y. Hirao, et al. (2003). "Interactions between the oocyte and surrounding somatic cells in follicular development: lessons from in vitro culture." $\underline{\mathrm{J}}$. Reprod. Dev. 49(4): 259-269.

Shi, F.-T., A. P. Cheung, et al. (2009). "Effects of endogenous growth differentiation factor 9 on activin A-induced inhibin B production in human granulosa-lutein Cells." J Clin Endocrinol Metab 94(12): 5108-5116. 
Shimasaki, S., R. K. Moore, et al. (2004). "The Bone Morphogenetic Protein System In Mammalian Reproduction." Endocr Rev 25(1): 72-101.

Shimasaki, S. (2006). "BMP-15 regulation of ovulation quota in mammals." Reproductive Medicine and Biology 5(4): 245-248.

Sicinski, P., J. L. Donaher, et al. (1996). "Cyclin D2 is an FSH-responsive gene involved in gonadal cell proliferation and oncogenesis." Nature 384(6608): $470-474$.

Sidis, Y., A. L. Schneyer, et al. (2001). "Follistatin: essential role for the N-terminal domain in activin binding and neutralization." Journal of Biological Chemistry 276(21): 17718-17726.

Silva, J. M., M. Hamel, et al. (2006). "Control of oestradiol secretion and of cytochrome $\mathrm{P} 450$ aromatase messenger ribonucleic acid accumulation by FSH involves different intracellular pathways in oestrogenic bovine granulosa cells in vitro." Reproduction 132: 909 - 917.

Silva, B. D. M., E. A. Castro, et al. (2010). "A new polymorphism in the growth and differentiation factor 9 (GDF9) gene is associated with increased ovulation rate and prolificacy in homozygous sheep." Animal Genetics: no-no.

Su, Y.-Q., X. Wu, et al. (2004). "Synergistic roles of BMP15 and GDF9 in the development and function of the oocyte-cumulus cell complex in mice: genetic evidence for an oocyte-granulosa cell regulatory loop." Developmental Biology 276(1): 64-73.

Su, Y.-Q., K. Sugiura, et al. (2008 (2007)). "Oocyte regulation of metabolic cooperativity between mouse cumulus cells and oocytes: BMP15 and GDF9 control cholesterol biosynthesis in cumulus cells." Development 135 (epub ahead of print): 111-121 (dev.009068). 
Sugiura, K. and J. J. Eppig (2005). "Society for reproductive biology founders' lecture 2005. Control of metabolic cooperativity between oocytes and their companion granulosa cells by mouse oocytes." Reproduction, Fertility and Development 17(7): 667-674.

Sugiura, K., Y.-Q. Su, et al. (2007). "Oocyte-derived BMP15 and FGFs cooperate to promote glycolysis in cumulus cells." Development 134(14): 2593-2603.

Sun, R., L. Lei, et al. (2010). "Expression of GDF-9, BMP-15 and their receptors in mammalian ovary follicles." Journal of Molecular Histology 41(6): 325-332.

Sutton, M. L., P. D. Cetica, et al. (2003). "Influence of oocyte-secreted factors and culture duration on the metabolic activity of bovine cumulus cell complexes." Reproduction 126(1): 27-34.

Thomas, F. H., J.-F. Ethier, et al. (2005). "Follicle-stimulating hormone regulates oocyte growth by modulation of expression of oocyte and granulosa cell factors." Endocrinology 146(2): 941-949.

Thompson, J. G., M. Lane, et al. (2007). Metabolism of the bovine cumulus-oocyte complex and influence on subsequent developmental competence. Reproduction in Domestic Ruminants VI Soc Reprod Fertil Suppl. J. L. Juengel, J. F. Murray and M. F. Smith. UKs, Nottingham University Press. 64: 179-190.

Ueno, N., N. Ling, et al. (1987). "Isolation and partial characterization of follistatin: a single-chain $\mathrm{Mr}$ 35,000 monomeric protein that inhibits the release of folliclestimulating hormone." Proceedings of the National Academy of Sciences 84(23): 8282-8286. 
Vaccari, S., K. Horner, et al. (2008). "Generation of mouse oocytes defective in cAMP synthesis and degradation: endogenous cyclic AMP is essential for meiotic arrest." Developmental Biology 316(1): 124-134.

van Wagenen, G. and M. E. Simpson (1965). Embryology of the ovary and testis Homo sapiens and Macaca mulatta. New Haven and London, Yale University Press.

Vanderhyden, B. C., P. J. Caron, et al. (1990). "Developmental pattern of the secretion of cumulus expansion-enabling factor by mouse oocytes and the role of oocytes in promoting granulosa cell differentiation." Developmental Biology 140(2): 307-317.

Vanderhyden, B. C., E. E. Telfer, et al. (1992). "Mouse oocytes promote proliferation of granulosa cells from preantral and antral follicles in vitro." Biology of Reproduction 46(6): 1196-1204.

Visser, J. A. and A. P. N. Themmen (2005). "Anti-Mullerian hormone and folliculogenesis." Molecular and Cellular Endocrinology 234(1-2): 81-86.

Vitt, U. A., M. Hayashi, et al. (2000). "Growth differentiation factor-9 stimulates proliferation but suppresses the follicle-stimulating hormone-induced differentiation of cultured granulosa cells from small antral and preovulatory rat follicles." Biol Reprod 62(2): 370-377.

Vitt, U. A., S. Mazerbourg, et al. (2002). "Bone morphogenetic protein receptor type II is a receptor for growth differentiation factor-9." Biology of Reproduction 67(2): 473-480.

Webb, R. and B. G. England (1982). "Relationship between LH receptor concentrations in thecal and granulosa cells and in-vivo and in-vitro steroid 
secretion by ovine follicles during the preovulatory period." J Reprod Fertil 66(1): 169-180.

Webb, R., P. C. Garnsworthy, et al. (2007). "Intra-ovarian regulation of follicular development and oocyte competence in farm animals." Theriogenology In Press, Corrected Proof.

Welt, C. K., Z. A. Smith, et al. (2001). "Differential regulation of inhibin A and inhibin B by luteinizing hormone, follicle-stimulating hormone, and stage of follicle development." Journal of Clinical Endocrinology Metabolism 86(6): $2531-2537$

Welt, C., Y. Sidis, et al. (2002). "Activins, inhibins, and follistatins: from endocrinology to signaling. A paradigm for the new millennium." Experimental Biology and Medicine 227(9): 724-752.

Wu, Y.-T., L. Tang, et al. (2007). "High bone morphogenetic protein-15 level in follicular fluid is associated with high quality oocyte and subsequent embryonic development." Human Reproduction 22(6): 1526-1531.

Xiao, S., D. Robertson, et al. (1992). "Effects of activin and follicle-stimulating hormone (FSH)-suppressing protein/follistatin on FSH receptors and differentiation of cultured rat granulosa cells." Endocrinology 131(3): 10091016.

Yan, C., P. Wang, et al. (2001). "Synergistic roles of bone morphogenetic protein 15 and growth differentiation factor 9 in ovarian function." Molecular Endocrinology 15(6): 854-866.

Yeager, M., V. M. Unger, et al. (1998). "Synthesis, assembly and structure of gap junction intercellular channels." Current Opinion in Structural Biology 8(4): 517-524. 
Yeo, C. X., R. B. Gilchrist, et al. (2008). "Exogenous growth differentiation factor 9 in oocyte maturation media enhances subsequent embryo development and fetal viability in mice." Human Reproduction 23(1): 67-73.

Yeo, C. X., R. B. Gilchrist, et al. (2009). "Disruption of bidirectional oocyte-cumulus paracrine signaling during in vitro maturation reduces subsequent mouse oocyte developmental competence." Biology of Reproduction 80(5): 10721080.

Yoshino, O., H. E. McMahon, et al. (2006). "A unique preovulatory expression pattern plays a key role in the physiological functions of BMP-15 in the mouse." PNAS 103(28): 10678-10683.

Zhao, H., Y. Qin, et al. (2007). "Analyses of GDF9 mutation in 100 Chinese women with premature ovarian failure." Fertility and Sterility 88(5): 1474-1476. 